\title{
Effective Coastal Boundary Conditions for Tsunami Simulations
}

\section{Wenny Kristina}


EFFECTIVE COASTAL BOUNDARY CONDITIONS FOR TSUNAMI SIMULATIONS

Wenny Kristina 


\section{Samenstelling promotiecommissie:}

Voorzitter en secretaris:

prof.dr.ir. P. M. G. Apers University of Twente

Promotor

prof.dr.ir. E. W. C. van Groesen University of Twente

prof.dr.ir. O. Bokhove University of Leeds, UK

\section{Leden}

prof.dr.ir. J. J. W. van der Vegt University of Twente

prof.dr.ir. H. W. M. Hoeijmakers University of Twente

prof.dr.ir. R. H. M. Huijsmans Delft University of Technology

prof.dr. J. G. M. Kuerten Eindhoven University of Technology

prof.dr.ir. E. H. van Brummelen Eindhoven University of Technology

The research presented in this thesis was carried out at the Applied Analysis and Computational Science (AACS) group, Departement of Applied Mathematics, University of Twente, Enschede, The Netherlands and Laboratorium Matematika Indonesia (LabMath-Indonesia), Bandung, Indonesia.

The work described in this thesis is supported by Nederlandse Organisatie voor Wetenschappelijk Onderzoek (NWO), under project "Nearshore Tsunami Modelling and Simulations", project number 817.01.014.

Copyright (C) 2014, Wenny Kristina, Enschede, The Netherlands.

Printed by Wöhrmann Print Service, Zutphen, The Netherlands.

ISBN 978-90-365-3755-1

DOI $10.3990 / 1.9789036537551$ 


\title{
EFFECTIVE COASTAL BOUNDARY CONDITIONS FOR TSUNAMI SIMULATIONS
}

\author{
PROEFSCHRIFT
}

ter verkrijging van

de graad van doctor aan de Universiteit Twente, op gezag van de rector magnificus, prof. dr. H. Brinksma, volgens besluit van het College voor Promoties, in het openbaar te verdedigen op donderdag 2 oktober 2014 om 14.45 uur

door

Wenny Kristina

geboren op 15 februari 1985

te Malang, Indonesië 
This dissertation has been approved by:

prof. dr. ir. E. W. C. van Groesen

prof. dr. ir. O. Bokhove 
this thesis is dedicated to my mother and the memory of my father to my husband 

Summary ix

Samenvatting $\quad$ xi

\begin{tabular}{lll}
\hline & Introduction & 1
\end{tabular}

1.1 Motivation ........................... 1

1.2 Effective Boundary Condition . . . . . . . . . . . . . . . . . 4

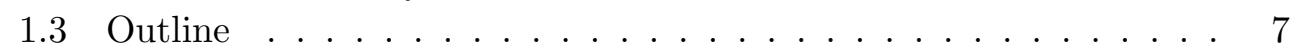

2 EBC for Dispersive Tsunami Propagation 9

2.1 Introduction . . . . . . . . . . . . . . . . . 10

2.2 Linear Variational Boussinesq Models . . . . . . . . . . . . . . . . 12

2.2 .1 Continuum case . . . . . . . . . . . . . . . . . . 12

2.2 .2 Semi-discrete case . . . . . . . . . . . . . . . . . 16

2.3 Effective Boundary Conditions: Shallow Water Equations . . . . . 18

$2.3 .1 \quad$ Flat Bottom Case -WKB0 . . . . . . . . . . . . . . . 18

2.3.2 WKB approximation -WKB1 \& WKB2 . . . . . . . . . . . 20

2.4 Modelling Effective Boundary Conditions: Boussinesq Equations

-DWKB0, DWKB1 \& DWKB2 . . . . . . . . . . . . 23

2.4 .1 Observation and influx operators . . . . . . . . . . . 24

2.5 Numerical Validation . . . . . . . . . . . . . . . . . . . . 27

2.5 .1 Flat Bottom Situation . . . . . . . . . . . . . . . 27

2.5.2 Slowly Varying Topography . . . . . . . . . . . . . . . . . . 30

2.5 .3 Periodic waves and wall reflections . . . . . . . . . . . . . 39

2.6 Conclusions . . . . . . . . . . . . . . . . . . . . . . . . . . . . . . . 40 
\begin{tabular}{|lll}
3 & EBC for Tsunami Wave Run-Up over Sloping Bathymetry & 47
\end{tabular}

3.1 Introduction $\ldots \ldots \ldots \ldots \ldots \ldots \ldots \ldots$

3.2 Water wave models . . . . . . . . . . . . . . . . . . . 51

3.3 Nonlinear Shallow Water Equations $\ldots \ldots \ldots \ldots$

3.3 .1 Characteristic form . . . . . . . . . . . . . . . 55

3.3.2 A trivial solution of the characteristic curve . . . . . . . . 57

$3.3 .3 \quad$ Boundary Value Problem (BVP) . . . . . . . . . . . 58

3.4 Effective Boundary Condition . . . . . . . . . . . . . . 62

$3.4 .1 \quad$ Finite element implementation . . . . . . . . . . . . 62

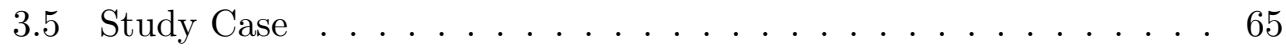

3.5.1 Solitary wave . . . . . . . . . . . . . 66

3.5 .2 Periodic wave . . . . . . . . . . . . . . . 71

3.5.3 Simulation using simplified Aceh bathymetry . . . . . . . . 74

3.6 Conclusions . . . . . . . . . . . . . . . . . 80

4 Conclusions and Outlook $\quad 85$

4.1 Conclusions . . . . . . . . . . . . . . . . . . . . . . . . . 85

4.2 Outlook $\ldots \ldots \ldots \ldots \ldots \ldots$

\begin{tabular}{ll}
\hline Bibliography & 89
\end{tabular}

\begin{tabular}{ll}
\hline Acknowledgments & 97
\end{tabular} 
Numerical modeling of tsunami propagation at the coastal zone has been a daunting task since high accuracy is needed to capture aspects of wave propagation in the more shallow areas. For example, there are complicated interactions between incoming and reflected waves due to the bathymetry, the run-up and run-down flooding phenomena at the beaches or (other) man-made structures that form the coastline, and intrinsically nonlinear phenomena of wave propagation. Numerical modeling of tsunamis with nested methods in shallower areas is computationally expensive and difficult to use in the operational practice. Meanwhile, if a fixed wall boundary condition is used at a certain shallow depth contour, the reflection properties can be unrealistic. To alleviate this, we develop a so-called effective boundary condition as a novel technique to predict tsunami wave run-up along the coast and offshore wave reflections.

The general idea of the effective boundary condition is as follows. From the deep ocean to a seaward boundary, i.e., in the simulation area, the wave propagation is modeled numerically over real bathymetry using either nondispersive, linear, shallow water equations or the dispersive, linear, variational Boussinesq model. The incoming wave is measured at this seaward boundary, and the wave dynamics towards the shoreline and the reflection caused by the bathymetry are modeled analytically. The reflected wave is then influxed back into the simulation area using the effective boundary condition. The location of this seaward boundary point is determined by assessing when nonlinearity starts to play a role in the wave propagation.

The modeling of wave dynamics towards the shoreline is achieved by employing the analytical solution of (i) linear shallow water equations and (ii) nonlinear shallow water equations. The linear approach is started with the simplest case, that is flat bathymetry with closed wall boundary condition. Further, a slowly 
varying bathymetry case is considered. The analytical solution is based on linear shallow water theory and the Wentzel-Kramer-Brillouin approximation, as well as extensions to the dispersive Boussinesq model. Subsequently, in the nonlinear approach, the coastal bathymetry is approximated by using a mean, planar beach. The run-up heights at the shore and the reflection caused by the slope are then modeled based on nonlinear shallow water theory over sloping bathymetry.

The coupling between the numerical and analytic dynamics in the two areas is handled using variational principles, which leads to (approximate) conservation of the overall energy in both areas. The numerical solution in the simulation area is based on a variational finite element method. Verifications of the effective boundary condition technique and implementation are done in a series of numerical test cases of increasing complexity, including a case akin to tsunami propagation to the coastline at Aceh, Sumatra, Indonesia. The comparisons show that the effective boundary condition method gives a good prediction of the wave arriving at the shoreline as well as the wave reflection, based only on the information of the wave signal at this seaward boundary point. The computational times needed in simulations using the effective boundary condition implementation show a reduction compared to times required for corresponding full numerical simulations. 


\section{Samenvatting}

Numerieke modellering van tsunami propagatie in de kustzone is een lastige taak sinds hoge nauwkeurigheid is nodig om aspecten van golfvoortplanting in de meer ondiepe gebieden vast te leggen. Er zijn bijvoorbeeld ingewikkelde interacties tussen inkomende en gereflecteerde golven als gevolg van de bathymetrie, de aan- en oploop met overstromingsverschijnselen op de stranden of (andere) door de mens gemaakte structuren die de kustlijn vormen, en intrinsiek niet-lineaire fenomenen van golfvoortplanting. Numerieke modellering van tsunami's met geneste methoden in ondiep water zijn computationeel duur en moeilijk te gebruiken in de praktijk. Daarnaast kunnen de reflectie-eigenschappen onrealistisch zijn als een vaste wand randvoorwaarde wordt gebruikt bij een bepaalde dieptecontour. Om dit te verbeteren ontwikkelen we een zogenaamde effectieve randvoorwaarde als een betere techniek om tsunami-oploop langs de kust en offshore golfreflecties te kunnen voorspellen.

Het algemene idee van de effectieve randvoorwaarde is als volgt. Vanaf de diepe oceaan naar een zeewaartse grens, dat wil zeggen, in de simulatie omgeving, wordt de golfvoortplanting numeriek gemodelleerd over echte bathymetrie met behulp van niet-dispersieve, lineaire, ondiep water vergelijkingen of het dispersieve, lineaire, variationele Boussinesq model. De inkomende golf wordt gemeten op deze zeewaartse grens, en de golfdynamiek in de richting van de kust en de reflectie veroorzaakt door de bathymetrie wordt analytisch gemodelleerd. De gereflecteerde golf wordt dan teruggekaatst in het simulatiegebied met behulp van de effectieve randvoorwaarde. De locatie van dit zeewaartse grenspunt wordt bepaald voordat de niet-lineariteit een belangrijke rol in de golfvoortplanting speelt.

Het modelleren van golfdynamica naar de kust wordt bereikt door gebruik van de analytische oplossing van in eerste instantie lineaire vergelijkingen voor ondiep 
water en in tweede instantie niet-lineaire vergelijkingen voor ondiep water. De lineaire benadering wordt gestart met het eenvoudigste geval, dat is een vlakke bathymetrie met een vaste wand als randvoorwaarde. Verder is een langzaam variërende bathymetrie beschouwd. De analytische oplossing is gebaseerd op lineaire ondiep water theorie en de Wentzel-Kramer-Brillouin benadering, evenals uitbreidingen voor het dispersieve Boussinesq model. Vervolgens worden in de lineaire benadering de waterdiepten aan de kust benaderd door een strand met een constante gemiddelde helling. De oploophoogten aan de kust en de reflectie veroorzaakt door de helling worden vervolgens gemodelleerd op basis van nietlineaire ondiep water theorie over hellende bathymetrie.

De koppeling tussen de numerieke en analytische dynamiek in de twee gebieden wordt behandeld met behulp variatieprincipes, wat bij benadering leidt tot behoud van de totale energie in beide gebieden. De numerieke oplossing in het simulatiegebied is gebaseerd op een variationele eindige elementenmethode. Verificaties van de effectieve randvoorwaarde techniek en implementatie worden gedaan in een reeks van numerieke testcases van toenemende complexiteit, waaronder een geval verwant aan tsunami-oploop naar de kustlijn op Atjeh, Sumatra, Indonesie. Bij vergelijking blijkt dat de effectieve randvoorwaarde methode een goede voorspelling geeft van zowel de golf die aankomt bij de kustlijn alsmede van de golfreflectie, terwijl deze methode uitsluitend is gebaseerd op de informatie van het golfsignaal bij dit zeewaartse grenspunt. De rekentijden die nodig zijn in simulaties met behulp van de effectieve randvoorwaarde tonen een reductie ten opzichte van tijden die nodig zijn voor overeenkomstige volledige numerieke simulaties. 


\section{CHAPTER 1}

\section{Introduction}

\subsection{Motivation}

The word tsunami is a Japanese word, represented by two characters: tsu, meaning, harbor, and nami, meaning, wave. Tsunami is defined as a set of ocean waves with very long wavelengths (typically hundreds of kilometres) and relatively small amplitude (a metre or so is typical), so that it often passes by ships in the deep ocean without anyone on board even noticing. The cause of a tsunami is any large, sudden disturbance of the sea-surface, such as an underwater earthquake, landslide, or volcanic eruption. More rarely, a tsunami can be generated by a giant meteor impact with the ocean. About 80 percent of tsunamis happen within the Pacific Ocean's "Ring of Fire", which is an active geological area where tectonic shifts make volcanoes and earthquakes common.

Historical data of tsunamis record that since 1850 tsunamis have killed more than 420,000 people and caused billions of dollars of damage to coastal structures and habitats [Bernard and Robinson, 2009]. Knowing no international boundaries across the sea, tsunami propagation is a problem with global dimensions and ranks high on the scale of natural disasters. Most of the casualties were caused by local tsunamis that occur about once per year somewhere in the world. The December 26, 2004 Indian Ocean Tsunami (IOT) with a Moment magnitude $(\mathrm{Mw})$ of 9.2 was likely the most devastating tsunami in recorded history, causing over 200,000 fatalities within a few hours in 27 countries across the entire Indian Ocean basin, with tens of thousands reported missing and over one million left homeless Kawata et al., 2005, Yalciner et al., 2005. 

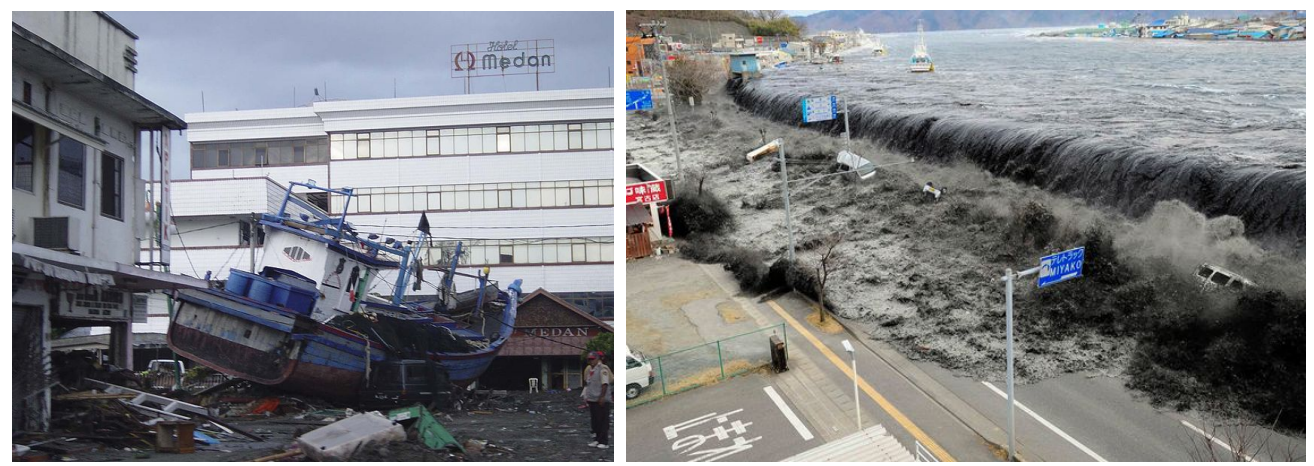

Figure 1.1: Left: Boat washed ashore near local businesses in down town Aceh, Sumatra, following a massive tsunami that struck the area on the $26^{\text {th }}$ of December 2004 (Courtesy of Michael L. Bak/ US Navy). Right: A tsunami wave crashes over a street in Miyako City, Iwate Prefecture, in northeastern Japan on March 11, 2011 (Courtesy of Mainichi Shimbun/ Reuters).

Following the IOT, there has been substantial interest in developing tsunami mitigation plans worldwide Synolakis and Bernard, 2006. Tsunami science and engineering are inevitable to save human society, industries, and natural environment. Fortunately, modern observational technologies such as the geographical information system (GIS), the global positioning system (GPS), and remote sensing techniques have enabled scientists to obtain data of seismic activity, sea floor bathymetry, topography, and wave height [Zhang et al., 2008]. Together with these data, another important task is to develop numerical models for more accurate and more reliable forecasting of tsunami propagation through vast oceans before they strike the coastlines [Meinig et al., 2005].

Since the wavelengths of tsunamis are far greater than the depth of the ocean, shallow water equations are widely used in the modeling of tsunamis [Choi et al. 2011, Liu et al., 2009]. With the simplicity of these equations, tsunami simulations and forecasting can be done in a short time. One feature of these equations is that the speed of propagation of tsunamis can be approximated by $c=\sqrt{g h}$, that is dependent upon the water depth $h$ and gravity acceleration $g=9.8 \mathrm{~m} / \mathrm{s}^{2}$. For example, the typical water depth in the deep ocean is around $4000 \mathrm{~m}$, so tsunamis will therefore travel at around $200 \mathrm{~m} / \mathrm{s}$ or more than $700 \mathrm{~km} / \mathrm{h}$, the speed of a passenger jet plane. But as they approach the shoreline and enter shallower water they slow down because of the shoaling due to the bathymetry. They begin to grow in height, and decrease in wavelength. It explains why a tsunami causes major disaster when it hits the shore.

During the IOT, multiple wave phenomena were observed throughout the Sri Lanka coastal area and along other coastlines; one witness said: "It wasn't one 
wave, it came in great surges, each one deeper than the last and pushing the water that had come in before in front of it." Horrillo et al., 2006. Analysis of the recorded data also shows this fact Kulikov, 2006. It indicates the presence of dispersion, that is the phenomena where wave components with different wavelengths travel at different propagation speeds. Saito et al. 2011 found that dispersion effects played a significant role as well in the Japan Tsunami (JT) of March 2011. In the numerical calculation of the 1993 Okushiri Island tsunami, Sato 1996] also found that local tsunami enhancement can be explained by a series of dispersive waves which ride on the main tsunami front. These dispersion phenomena will eventually deform the initial wave shape to multiple waves tailgating the main wave, and the effect can be significant Liu et al., 1995, Heinrich et al. 1998.

In tsunami calculations, the dispersion effects are usually described through Boussinesq approximations [Madsen et al., 1991, Kennedy and Kirby, 2003]. Grilli et al. 2007 and Horrillo et al. 2012 show differences of the wave elevations between shallow water and Boussineq approximations for IOT and North Pacific tsunami simulations. Figure 1.2 displays a snapshot of solitary wave propagation using the shallow water equations (solid line) and the Boussinesq model (dashed line).

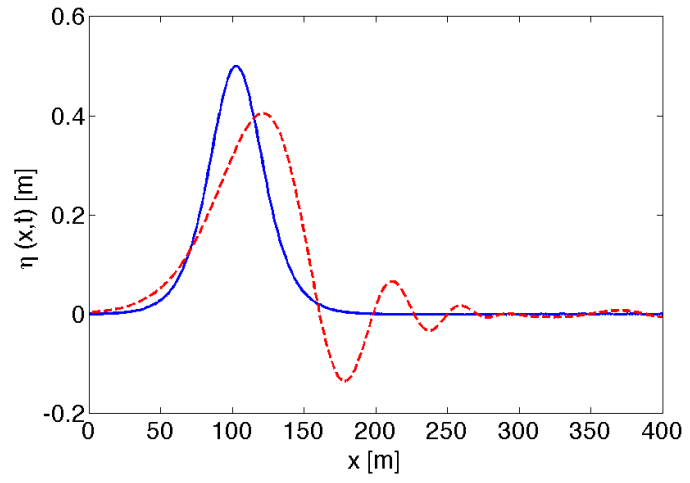

Figure 1.2: Snapshot of a soliton wave propagation using the shallow water model (solid line) and Boussinesq approximation (dashed line). The shallow water equations as the non-dispersive model retains the initial wave shape, whereas the dispersive model produces some successive wave tails behind the main one. The comparison also displays that the non-dispersive model produces a higher leading wave amplitude compared to the dispersive one, as is the case in Ortiz et al. [2001] and Mader 2004].

The shallow water and Boussinesq equations are obtained from the parent Navier-Stokes equations. A numerical study of the IOT using these three different models has been carried out by Horrillo et al. [2006]. They utilize the Navier-Stokes equations to provide a frame of reference in validating the shallow water and Boussinesq equations in a (horizontally) one-dimensional channel case. Nevertheless, the Navier-Stokes equations are presently too computationally intensive for inundation mapping or operational forecasting, and are generally used 
for free-surface flows of very limited geographical extent Synolakis et al., 2008. In this thesis, both the shallow water and the Boussinesq equations will be used to simulate the tsunamis and a comparison of these two models will be presented.

It has been mentioned before that another characteristic of tsunamis is that they often are of low-amplitude as one long wave at their origin. But as they approach the shoreline and enter shallower water they begin to grow in height and decrease in wavelength because of the shoaling due to the bathymetry. A direct calculation of tsunami propagation from its source to the coastal zone using a single numerical model results in low accuracy in its estimation of tsunami runup height. In order to capture these shoaling effects in more detail, smaller grid sizes are therefore needed in the numerical models. Some numerical models of tsunamis use nested methods with different mesh resolution to preserve the accuracy of the solution in the coastal area [Wei et al., 2008, Roger et al., 2010, Titov et al., 2011]. Consequently, longer computational times are then usually required. Other models often employ an impenetrable vertical wall or transparent boundary at a certain depth contour as the boundary condition Zaibo et al., 2003, Wang and Liu, 2006. Obviously, the reflection properties of such a boundary condition can be unrealistic. The main goal of this thesis is therefore the wish to alleviate this shortcoming by an investigation of a so-called effective boundary condition (EBC).

\subsection{Effective Boundary Condition}

A two-dimensional cross-section of the ocean is shown in Fig. 1.3. The effective boundary condition (EBC) is assigned at a certain shallow depth contour in the coastal zone (this will be referred to as the seaward boundary afterward). The general idea of the EBC is as follows:

- From the deep ocean to the seaward boundary, i.e., in the simulation area, the wave propagation is modeled numerically over realistic bathymetry using either the linear shallow water equations (LSWE) or the linear variational Boussinesq model (LVBM).

- The incoming wave at this seaward boundary is measured (in time), and the wave dynamics towards the shoreline is modeled analytically (using suitable approximations).

- The reflected wave is then influxed back into the simulation area using the EBC. 


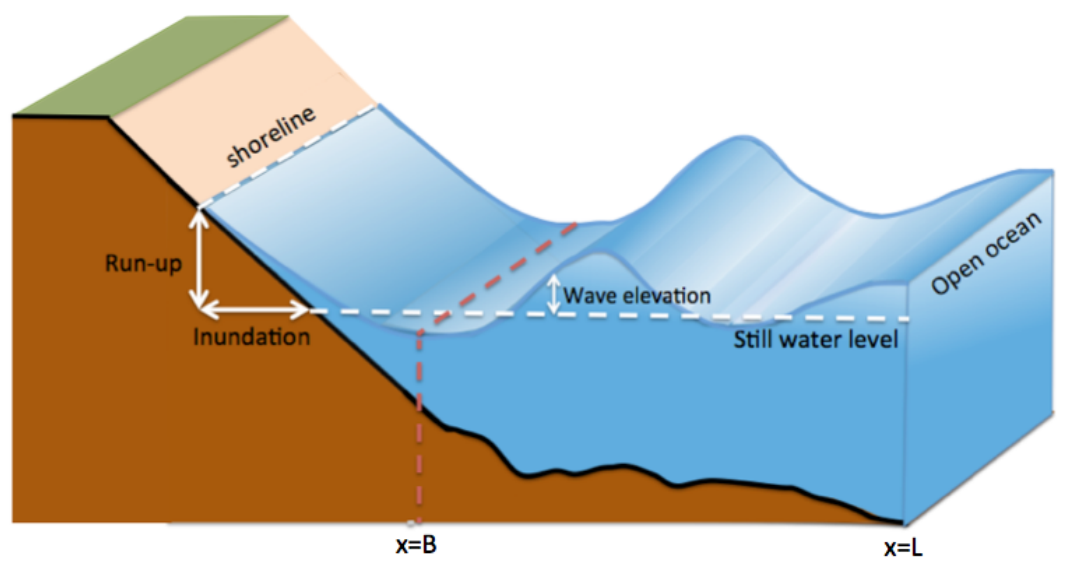

Figure 1.3: Sketch of a two-dimensional cross-section of the ocean. At the seaward boundary $x=B$, we measure the wave elevation and velocity, and we want to find a solution of the nonlinear shallow water equations in the sloping region near the shoreline.

By implementing this EBC technique, the forecasting of the tsunami propagation and run-up can be done in a shorter time without losing the accuracy since the coastal area is modeled analytically.

A rapid method to estimate tsunami run-up heights is also suggested by Choi et al. (2011, 2012) by integrating two-dimensional (2D) shallow water model and an analytical one-dimensional (1D) long-wave run-up theory. A hard-wall boundary condition is imposed at the seaward boundary and the water wave oscillations at this boundary line are measured. The maximum run-up height of tsunami waves at the coast is subsequently calculated separately by employing a linear approach. In addition to calculating only the maximum run-up height, the EBC also includes the calculation of reflected waves. Thus, the point-wise wave height in the whole domain (offshore and onshore area) is predicted accurately. It is necessary to take into account the interaction between incoming and reflected waves since subsequent waves may cause danger at later times. Stefanakis et al. 2011] discover that resonant phenomena between the incident wavelength and the beach slope occur. The resonance happens due to incoming and reflected wave interactions. The October 25, 2010 Mentawai Islands tsunami is studied and multiple resonant frequencies are observed in this case. Ezersky et al. 2013] also study the resonant effects for conditions near the Indian coast where the 1945 Makran tsunami was recorded. It is shown that the incident waves are amplified more than 10 times higher in the onshore region due to the resonant phenomena.

For reasons of simplicity and clarity of exposure, the EBC in this thesis is derived in a $1 \mathrm{D}$ approach in the horizontal. The integration of $2 \mathrm{D}$ numerical 
modeling in the simulation area with 1D analytical calculations in the coastal area can be extended directly under certain limitations. Since a 1D model is employed in the nearshore area, the 2D effects such as refraction, focusing, etc., are not included in the model. Furthermore, near-shore wave breaking of tsunami waves is also ignored in the present analytical solution of nonlinear shallow water equations. For waves incident at a small angle to the beach normal, the onshore problem can be calculated using the analytical 1D run-up theory of the nonlinear model, and independently the longshore velocity can be computed asymptotically by using the approach of Ryrie [1983]. By using 2D numerical modeling in the open sea towards the seaward boundary line and employing this approach in the nearshore area, in principle the EBC method can be applied as well in two horizontal dimensions. Such an approach is expected to be approximately valid for 2D flow with slow variations along the EBC line.

The shallow water equations (both linear and nonlinear) and Boussinesq model will be derived here via the variational formulation for surface water waves of Miles 1977. The numerical solution in the simulation area is based on a variational finite element method (FEM). The coupling condition between the two areas is also handled using the variational principles, which leads to (approximate) conservation of the overall energy in both areas. This condition is required when the simulation area is approximated with a finite element approximation yet the nearshore area stays continuous.

The location of the seaward boundary is determined as the point before the effect of nonlinearity arises, and we examine the dispersion effect at that point as well. Considering the KdV equation [Mei, 1989], the measures of nonlinearity and dispersion are given by the ratios $\delta=A / h$ and $\mu^{2}=(k h)^{2}$, for the wave amplitude $A$, water depth $h$, and wavenumber $k$. Provided with the information of the initial wave profile, we can calculate the amplification of the amplitude and the decrease of the wavelength in a linear approach, and thereafter estimate the location of the EBC point a priori before the arrival of the wave.

Given the above summary and discussion, the goals in this thesis are as follows:

(i) To formulate the effective boundary conditions for the linear shallow water equations (LSWE) and the linear variational Boussinesq model (LVBM).

(ii) To integrate such effective boundary conditions with a finite element treatment in the simulation area and analytical solution in the model area, by using variational principles.

(iii) To verify our approach in a series of numerical test cases of increasing complexity. 


\subsection{Outline}

In the previous section, the background and goals of this thesis have been described. Subsequently, the Effective Boundary Condition (EBC) technique was introduced in a pedagogical fashion. In this short section, we present the contents of the rest of this thesis.

In Chapter 2, the LSWE and LVBM are derived from their variational principles. In addition, the coupling conditions required at the seaward boundary point, where the simulation area is approximated with a finite element approximation yet the model area stays continuous, are also derived. This was done in a $1 \mathrm{D}$ setting, but the derivation of these coupling conditions in 2D are straightforward and similar. The EBC formulation is started with the simplest case, that is for flat and slowly varying bathymetry in the nearshore area. The analytical solution is obtained by utilizing linear shallow water theory and the WentzelKramer-Brillouin (WKB) approximation Bremmer, 1951, Bender and Orszag, 1978, van Groesen and Molenaar, 2007). Numerical verifications are shown for various test cases.

Chapter 3 naturally follows the contents of Chapter 2. Furthermore, the EBC that includes run-up phenomena is formulated by approximating the bathymetry as a planar beach. The shoreline position and wave reflection in the model area (sloping region) are determined using an analytical solution of the nonlinear shallow water equations (NSWE) following the approach of Antuono and Brocchini [2010] for unbroken waves.

Finally, conclusions and an outlook for future work are given in Chapter 4. 



\section{Effective Coastal Boundary Conditions for Dispersive Tsunami Propagation}

\section{Abstract 1}

We aim to improve the techniques to predict tsunami wave heights along the coast. The modeling of tsunamis with the shallow water equations has been very successful, but often shortcomings arise, for example because wave dispersion is neglected. To bypass the latter shortcoming, we use the (linearized) variational Boussinesq model derived by Klopman et al. 2010 and compare its results with the shallow water model. Another shortcoming is that complicated interactions between incoming and reflected waves near the shore are usually simplified by a fixed wall or absorbing boundary condition at a certain shallow depth contour. To alleviate this, we explore and present a so-called effective boundary condition (EBC), developed here in one spatial dimension. From the deep ocean to a seaward boundary, i.e., in the simulation area, we model wave propagation numerically. We measure the incoming wave at this seaward boundary, and model the wave dynamics towards the shoreline analytically, based on shallow water theory and the Wentzel-Kramer-Brillouin (WKB) approximation, as well as extensions to the dispersive, Boussinesq model. The coupling between the

\footnotetext{
${ }^{1}$ This chapter is a revised version of an article submited to the journal Theor. Comp. Fluid Dyn.
} 
dynamics in the two areas, respectively treated numerically and analytically, is handled using variational principles, which leads to (approximate) conservation of the overall energy in both areas. The reflected wave is then influxed back into the simulation area using the $\mathrm{EBC}$ in a discrete, variational, finite element formulation. We verify our approach in a series of numerical test cases of increasing complexity, including a case akin to tsunami propagation to the coastline at Aceh, Sumatra, Indonesia.

\section{$2.1 \quad$ Introduction}

The propagation of surface waves over the oceans and in harbors concerns motion that is largely inviscid, irrotational, and incompressible. The velocity is captured well with a velocity potential and is divergence free. Such water wave motion is governed adequately by Laplace's equation for this velocity potential coupled to dynamic and kinematic equations for the free surface dynamics Luke, 1967, Miles, 1977, Whitham, 1997]. The equations in this classical problem are fully nonlinear due to the nonlinear free surface boundary conditions, but can be linearized around a sea state of rest for waves of small amplitude. We are interested in the propagation of tsunamis to the coast and long waves into harbors in deeper water, and will therefore limit ourselves to linear wave theory.

Tsunami propagation in the deep ocean is classically calculated with even simpler equations than potential wave theory: the well-known shallow water equations Gill, 1982, Imamura et al., 2006]. It turns out that the lack of dispersion is a shortcoming of (linear) shallow water modeling. During the initial propagation, waves separate into spectral components with different frequencies and amplitudes. The leading wave is followed by a train of waves formed in its tail. Mader [1974] showed that the shallow water equations often failed to adequately resolve shorter wavelength tsunamis. Since the potential flow model is much more complicated than the shallow water model, Boussinesq approximations play an important role as simpler, more manageable, dispersive wave models of intermediate complexity, e.g., Shi et al., 2011, Kirby, 1997, Madsen et al., 1991, Peregrine, 1967]. Furthermore, we have recently advocated the use of variational or Hamiltonian Boussinesq models Klopman et al., 2010, 2005 because such models inherit the original geometric structure of the potential flow equations or even the incompressible Euler equations Cotter and Bokhove, 2010]. One of our goals is to show the strength of such variational, dispersive Boussinesq models.

Numerical models of tsunami propagation from the location of generation to widespread, surrounding coastlines must deal with details in the generation region, the proper large-scale long-wave propagation across the oceans to the coast, 


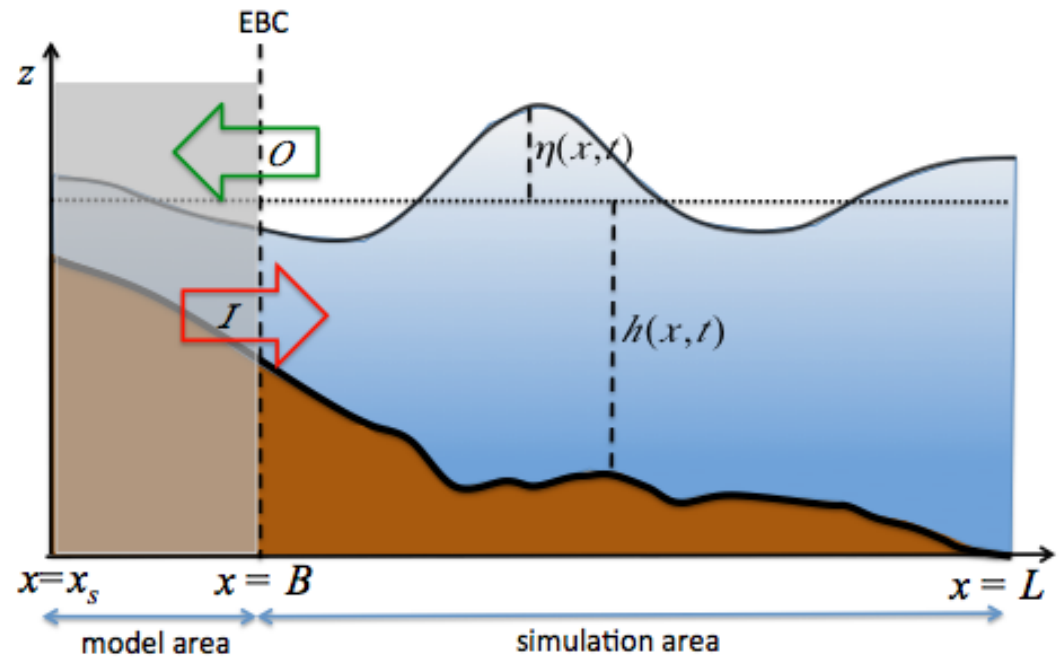

Figure 2.1: Illustration of the domain decomposition for our effective boundary treatment. The water depth at rest is given by $h=h(x)$ and the free surface elevation by $\eta=\eta(x, t)$ with horizontal coordinate $x$ and time $t$. At a position $x=B$ of given, nonzero depth, information of the incoming wave is determined in time. A theoretical model is used to obtain the wave reflection within a model area $x \in\left[x_{\mathrm{s}}, B\right]$. The information that accounts for these reflected waves is used into a simulation area $x \in[B, L]$ as an effective boundary condition at the point $x=B$.

and the subsequent fine-scale run-up and run-down flooding phenomena at the beaches, cliffs and man-made structures that form the coastline. It is a daunting task for numerical models to capture such a variation in spatial and temporal scales. Some shallow water tsunami models therefore approximate the coastline, or large tracts thereof, with an impenetrable vertical wall at a certain depth contour, say 20m (Zaibo et al. 2003]). Obviously, the reflection properties of such a boundary condition can be unrealistic. We wish to alleviate this shortcoming by an investigation of so-called effective boundary conditions, instead of these solid-wall boundary conditions. In one horizontal spatial dimension, an outline of the desired mathematical modeling is sketched in Fig. 2.1. In the deep ocean for $x \in[B, L]$ with horizontal coordinate $x$ an internal boundary point $B$, denoted as the simulation area, we model the wave propagation numerically. In the coastal zone for $x \in\left[x_{\mathrm{s}}, B\right]$ with end point $x_{\mathrm{s}}<B$, denoted as the model area, we model the wave propagation analytically and often approximately because sufficient numerical resources are lacking in order to capture its solution numerically. Of course, such lack of resources is really only a problem in two horizontal dimensions. 
Given the above summary and discussion, the goals in the present paper are as follows:

(i) To formulate effective boundary conditions (abbreviated as EBC) for the linear shallow water equations (LSWE) and the linear variational Boussinesq model (LVBM), based on well-known shallow water theory and the Wentzel-Kramer-Brillouin approximation (WKB), and extensions.

(ii) To integrate such effective boundary conditions with a finite element treatment in the simulation area, and analytical, asymptotic methods in the model area, by using variational principles. One reason to do so, is that this approach leads in principle to a compatible numerical scheme with global energy conservation in the entire simulation and model area (given suitable boundary conditions at the external boundaries at $x=x_{\mathrm{s}}$ and $x=L$ ) for the flat bottom case and the leading order WKB approximation.

(iii) To verify our approach in a series of numerical test cases of increasing complexity.

Our methodology will be derived in one spatial dimension for reasons of simplicity and clarity of exposure.

The outline naturally arises from the above goals. In $\$ 2.2$, we introduce the linear shallow water equations (LSWE) and the linear variational Boussinesq model (LVBM) from their variational principles. In addition, we derive the coupling conditions required when the "simulation area" is approximated with a finite element approximation yet the "model area" stays continuous. In 2.3 and $\$ 2.4$, effective boundary conditions are derived. It is required to pinpoint the coupling conditions derived between the finite element simulation area and the model area. Classical linear wave theory for shallow water over a flat bottom and a WKB approximation over slowly varying topography is applied and extended to the present application. Numerical verifications are shown in \$2.5, and we conclude in $\$ 2.6$.

\subsection{Linear Variational Boussinesq Models}

\subsubsection{Continuum case}

Our primary goal is to model the water wave motion in the shallow water close to the shore analytically, instead of resolving the motion in these shallow regions numerically. We therefore introduce an artificial, open boundary at some depth and wish to determine an effective boundary condition at this boundary. To wit, for motion in a vertical plane normal to the shore with one horizontal dimension, 
this artificial boundary is placed at $x=B, x \in\left[x_{\mathrm{S}}(t), L\right]$, while the real (timedependent) boundary lies at $x=x_{\mathrm{s}}(t)$ with $x_{\mathrm{s}}(t)<B$, and time $t$. For example, land starts where the depth $h=h(x, t)=0$ at $x=x_{\mathrm{s}}(t)$. In general, this water line is time dependent when the wave moves up and down the beach. After linearization around a rest depth, $x_{\mathrm{s}}(t)=x_{\mathrm{s}}$ becomes fixed. To accommodate an analytical treatment to find an effective boundary condition, at $x=B$, we introduce several simplifications.

Firstly, we will restrict attention to the dynamics in a vertical plane with horizontal and vertical coordinates $x$ and $z$. Nonlinear, potential flow water waves are succinctly described by variational principles of Luke [1967] and Miles [1977]. Linear counterparts of these principles are readily stated as

$$
0=\delta \int_{0}^{T} \mathcal{L}[\phi, \Phi, \eta] \mathrm{d} t=\delta \int_{0}^{T} \int_{x_{\mathrm{s}}}^{L}\left(\phi \partial_{t} \eta-\frac{1}{2} g \eta^{2}-\int_{-h}^{0} \frac{1}{2}|\nabla \Phi|^{2} \mathrm{dz}\right) \mathrm{d} x \mathrm{~d} t
$$

with velocity potential $\Phi=\Phi(x, z, t)$, potential $\phi(x, t)=\Phi(x, 0, t)$ at the approximate location $z=0$ of the free surface, and the deviation $\eta=\eta(x, t)$ from this free surface at rest. Time runs from $t \in[0, T]$, the rest depth $h=h(x)$, partial derivatives are denoted by $\partial_{t}$ et cetera, the gradient in the vertical plane as $\nabla=\left(\partial_{x}, \partial_{z}\right)^{T}$, and the acceleration of gravity is $g$.

Secondly, following Klopman et al. [2010], we approximate the velocity potential as follows

$$
\Phi(x, z, t)=\phi(x, t)+F(z) \psi(x, t)
$$

for a function $F=F(z)$. Its suitability is determined by insisting that $F(0)=0$ such that $\phi$ is the potential at the approximate location $z=0$ of the free surface, and that the slip flow condition at the bottom boundary $z+h(x)=0$. The latter kinematic condition yields $\partial_{z} \Phi+\partial_{x} \Phi \partial_{x} h=0$ at $z=-h(x)$. For slowly varying bottom topography, this condition is approximated to

$$
\left(\partial_{z} \Phi\right)_{z=-h(x)}=F^{\prime}(-h) \psi=0 .
$$

Substitution of 2.2 into 2.1 yields the variational principle for the linearized Boussinesq equations

$$
\begin{aligned}
0 & =\delta \int_{0}^{T} \mathcal{L}[\phi, \psi, \eta] \mathrm{d} t \\
& =\delta \int_{0}^{T} \int_{x_{\mathrm{s}}}^{L}\left(\phi \partial_{t} \eta-\frac{1}{2} g \eta^{2}-\frac{1}{2} h\left(\partial_{x} \phi\right)^{2}-\beta \partial_{x} \psi \partial_{x} \phi-\frac{1}{2} \alpha\left(\partial_{x} \psi\right)^{2}-\frac{1}{2} \gamma \psi^{2}\right) \mathrm{d} x \mathrm{~d} t
\end{aligned}
$$


where functions $\beta(x), \alpha(x)$, and $\gamma(x)$ are given by

$$
\beta(x)=\int_{-h(x)}^{0} F \mathrm{~d} z, \quad \alpha(x)=\int_{-h(x)}^{0} F^{2} \mathrm{~d} z, \quad \text { and } \quad \gamma(x)=\int_{-h(x)}^{0}\left(F^{\prime}\right)^{2} \mathrm{~d} z .
$$

We choose a parabolic profile function $F(z ; h)=2 z / h+z^{2} / h^{2}$, in which the $x$ dependence is considered to be parametric when rest depth $h$ is sufficiently slowly varying such that in scaled form $h=h(\epsilon x)$ with $\epsilon \ll 1$ the ratio between vertical and horizontal changes in the topography. Consequently,

$$
\alpha=\frac{8}{15} h(x), \quad \beta=-\frac{2}{3} h(x), \quad \gamma=\frac{4}{3 h(x)} .
$$

Thirdly, when we ignore the underlined terms in (2.4), so for $\beta=\alpha=\gamma=0$, the linearized shallow water equations are obtained as limiting system. The advantage of the shallow water equations is that these permit exact or asymptotic solutions on flat and slowly varying bottom topography, respectively.

Next, we take variations of (2.4) but a priori divide the domain into two intervals, $x \in\left[x_{\mathrm{s}}, B\right]$ and $x \in[\overrightarrow{B, L}]$, such that we are forced to consider the boundary conditions that couple these two intervals. The resulting equations should, of course, equal the equations emerging when directly considering the entire domain $x \in\left[x_{\mathrm{s}}, L\right]$. The result of such variations, while using endpoint conditions $\delta \eta(x, 0)=\delta \eta(x, T)=0$, is

$$
\begin{aligned}
& 0=\int_{0}^{T}\left[\int_{x_{\mathrm{s}}}^{B}\right.\left(\left(\partial_{t} \eta+\partial_{x}\left(h \partial_{x} \phi\right)+\partial_{x}\left(\beta \partial_{x} \psi\right)\right) \delta \phi-\left(\partial_{t} \phi+g \eta\right) \delta \eta\right. \\
&\left.+\left(\partial_{x}\left(\alpha \partial_{x} \psi\right)+\partial_{x}\left(\beta \partial_{x} \phi\right)-\gamma \psi\right) \delta \psi\right) \mathrm{d} x \\
&-\left.\left(h \partial_{x} \phi+\beta \partial_{x} \psi\right)\right|_{x=B^{-}} \delta \phi_{x=B^{-}}-\left.\left.\left(\alpha \partial_{x} \psi+\beta \partial_{x} \phi\right)\right|_{x=B^{-}} \delta \psi\right|_{x=B^{-}} \\
&+\left.\left.\left(h \partial_{x} \phi+\beta \partial_{x} \psi\right)\right|_{x=x_{\mathrm{s}}} \delta \phi\right|_{x=x_{\mathrm{s}}}+\left.\left.\left(\alpha \partial_{x} \psi+\beta \partial_{x} \phi\right)\right|_{x=x_{\mathrm{s}}} \delta \psi\right|_{x=x_{\mathrm{s}}} \\
&+\int_{B}^{L}\left(\left(\partial_{t} \eta+\partial_{x}\left(h \partial_{x} \phi\right)+\partial_{x}\left(\beta \partial_{x} \psi\right)\right) \delta \phi-\left(\partial_{t} \phi+g \eta\right) \delta \eta\right. \\
&\left.+\left(\partial_{x}\left(\alpha \partial_{x} \psi\right)+\partial_{x}\left(\beta \partial_{x} \phi\right)-\gamma \psi\right) \delta \psi\right) \mathrm{d} x \\
&+\left.\left.\left(h \partial_{x} \phi+\beta \partial_{x} \psi\right)\right|_{x=B^{+}} \delta \phi\right|_{x=B^{+}}+\left.\left.\left(\alpha \partial_{x} \psi+\beta \partial_{x} \phi\right)\right|_{x=B^{+}} \delta \psi\right|_{x=B^{+}} \\
&\left.-\left.\left(h \partial_{x} \phi+\beta \partial_{x} \psi\right)\right|_{x=L} \delta \phi_{x=L}-\left.\left.\left(\alpha \partial_{x} \psi+\beta \partial_{x} \phi\right)\right|_{x=L} \delta \psi\right|_{x=L}\right] \mathrm{d} t
\end{aligned}
$$




$$
\begin{gathered}
\int_{0}^{T}\left[\int _ { x _ { \mathrm { s } } } ^ { B } \left(\left(\partial_{t} \eta+\partial_{x}\left(h \partial_{x} \phi\right)+\partial_{x}\left(\beta \partial_{x} \psi\right)\right) \delta \phi-\left(\partial_{t} \phi+g \eta\right) \delta \eta\right.\right. \\
\left.+\left(\partial_{x}\left(\alpha \partial_{x} \psi\right)+\partial_{x}\left(\beta \partial_{x} \phi\right)-\gamma \psi\right) \delta \psi\right) \mathrm{d} x \\
+\int_{B}^{L}\left(\left(\partial_{t} \eta+\partial_{x}\left(h \partial_{x} \phi\right)+\partial_{x}\left(\beta \partial_{x} \psi\right)\right) \delta \phi-\left(\partial_{t} \phi+g \eta\right) \delta \eta\right. \\
\left.\left.+\left(\partial_{x}\left(\alpha \partial_{x} \psi\right)+\partial_{x}\left(\beta \partial_{x} \phi\right)-\gamma \psi\right) \delta \psi\right) \mathrm{d} x\right] \mathrm{d} t
\end{gathered}
$$

The two boundary terms at each location $x=x_{\mathrm{s}}, x=B^{ \pm}$and $x=L$ concern the velocity across the depth. In the original variational principle (2.1), this would be $\nabla \Phi \cdot \hat{n}$ as a function of $z$ with $\hat{n}$ the outward normal at the respective vertical boundary, here $\hat{n}= \pm(1,0)^{T}$. For a hard wall at $x=L$, for example, the horizontal velocity must be zero at every depth. Due to the approximation (2.2), only two degrees of freedom over depth remain. The variations result thus in two boundary conditions at each location $x=x_{\mathrm{s}}, x=B^{ \pm}$and $x=L$. Assuming continuity at $x=B$, the boundary terms there cancel pairwise such that

$$
\begin{gathered}
\left.\left.\left(h \partial_{x} \phi+\beta \partial_{x} \psi\right)\right|_{x=B^{-}} \delta \phi\right|_{x=B^{-}}-\left.\left.\left(h \partial_{x} \phi+\beta \partial_{x} \psi\right)\right|_{x=B^{+}} \delta \phi\right|_{x=B^{+}}=0 \\
\left.\left.\left(\alpha \partial_{x} \psi+\beta \partial_{x} \phi\right)\right|_{x=B^{-}} \delta \psi\right|_{x=B^{-}}-\left.\left.\left(\alpha \partial_{x} \psi+\beta \partial_{x} \phi\right)\right|_{x=B^{+}} \delta \psi\right|_{x=B^{+}}=0
\end{gathered}
$$

The boundary terms at $x=x_{\mathrm{s}}, L$ are either zero for a solid wall, or transparent for the outgoing characteristic. Finally, given such boundary conditions and that the variations $\delta \phi, \delta \psi, \delta \eta$ are arbitrary in $2.7 \mathrm{~b}$, we find the equations:

$$
\begin{aligned}
\partial_{t} \phi+g \eta & =0, \\
\partial_{t} \eta+\partial_{x}\left(h \partial_{x} \phi\right)+\underline{\partial_{x}\left(\beta \partial_{x} \psi\right)} & =0, \\
\underline{\partial_{x}\left(\alpha \partial_{x} \psi\right)+\partial_{x}\left(\beta \partial_{x} \phi\right)-\gamma \psi} & =0
\end{aligned}
$$

for $x \in\left[x_{\mathrm{s}}, B\right]$ and $x \in[B, L]$. For the above choice of $F$, constant depth $h$, wave frequency $\omega$ and wave number $k$, the dispersion relation following from (2.9a) is given by

$$
\omega^{2}=g h k^{2}\left[1-\frac{\beta^{2} k^{2}}{h\left(\alpha k^{2}+\gamma\right)}\right] .
$$

Without the underlined terms, the linear shallow water equations remain as limiting system, without wave dispersion as we note from (2.10). Dispersion diagrams for the full potential flow, the linear variational Boussinesq model (LVBM), and the linear shallow water equations (LSWE) are shown in Fig. 2.2. The LSWE dispersion relation (dotted-dashed line) is a linear relation between $\omega$ and $k$, 


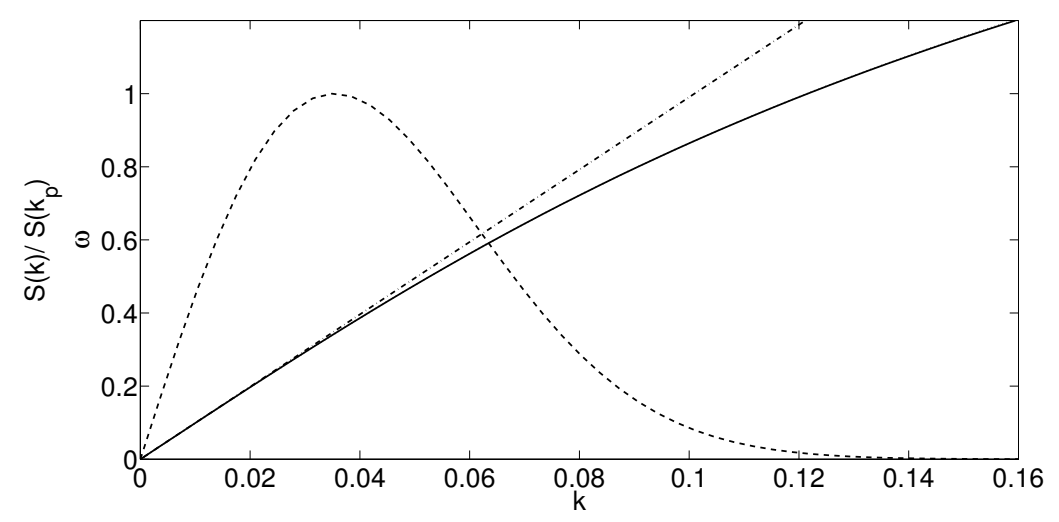

Figure 2.2: We combined the scaled spectrum (dashed line) of an initial profile in Fig 2.5, the exact dispersion relation for potential flow and LVBM dispersion relation (solid and dotted lines are on top of one another), and LSWE dispersion relation (dotteddashed line) in a plot of frequency $\omega$ versus wave number $k$.

showing that each wave number will travel with the same constant speed $\sqrt{g h}$. The LVBM dispersion relation 2.10 and the exact dispersion relation for potential flow (dotted line and solid line respectively) are on top of one another, showing that the long waves of present interest can be modeled well by LVBM. The dashed line is a scaled spectrum of an initial profile that will be used in Section 2.5. Each wave number will travel with its own speed following from the dispersion relation.

\subsubsection{Semi-discrete case}

The region $x \in[B, L]$ will be approximated using a classical Galerkin finite element expansion. We will use first order spline polynomials on $N=N_{k}$ elements with $j=1, \ldots, N+1$ nodes. The variational structure is simply preserved by substituting the expansions

$$
\phi_{h}(x, t)=\phi_{j}(t) \varphi_{j}(x), \quad \psi_{h}(x, t)=\psi_{j}(t) \varphi_{j}(x) \quad \text { and } \quad \eta_{h}(x, t)=\eta_{j}(t) \varphi_{j}(x)
$$

into (2.4) for $x \in[B, L]$ concerning $N_{k}$-elements and $(N+1)$-basis functions $\varphi_{j}$. We used the Einstein summation convention for repeated indices. 
To ensure continuity and a unique determination, we substitute

$$
\begin{aligned}
\phi(x, t) & =\tilde{\phi}(x, t)+\phi_{1}(t) \varphi_{1}(x), \\
\psi(x, t) & =\tilde{\psi}(x, t)+\psi_{1}(t) \varphi_{1}(x), \\
\eta(x, t) & =\tilde{\eta}(x, t)+\eta_{1}(t) \varphi_{1}(x),
\end{aligned}
$$

with $\varphi_{1}$ the basis function in element 0 for $x \in\left[x_{\mathrm{s}}, B\right]$ and with $\tilde{\phi}(B, t)=\tilde{\eta}(B, t)=$ $\tilde{\psi}(B, t)=0$. For linear polynomials, use of (2.11) into (2.4) yields

$$
\begin{array}{r}
0=\delta \int_{0}^{T}\left[M_{k l} \phi_{k} \dot{\eta}_{l}-\frac{1}{2} g M_{k l} \eta_{k} \eta_{l}-\frac{1}{2} S_{k l} \phi_{k} \phi_{l}-\frac{1}{2} A_{k l} \psi_{k} \psi_{l}-B_{k l} \psi_{k} \phi_{l}-\frac{1}{2} G_{k l} \psi_{k} \psi_{l}+\right. \\
\left.\int_{x_{\mathrm{s}}}^{B}\left(\phi \partial_{t} \eta-\frac{1}{2} g \eta^{2}-\frac{1}{2} h\left(\partial_{x} \phi\right)^{2}-\frac{1}{2} \alpha\left(\partial_{x} \psi\right)^{2}-\beta\left(\partial_{x} \phi\right)\left(\partial_{x} \psi\right)-\frac{1}{2} \gamma \psi^{2}\right) \mathrm{d} x\right] \mathrm{d} t
\end{array}
$$

$$
\begin{array}{r}
=\int_{0}^{T}\left[\left(M_{k l} \dot{\eta}_{l}-S_{k l} \phi_{l}-B_{k l} \psi_{l}\right) \delta \phi_{k}-\left(M_{k l} \dot{\phi}_{k}+g M_{k l} \eta_{k}\right) \delta \eta_{l}-\left(A_{k l} \psi_{l}+B_{k l} \phi_{l}+G_{k l} \psi_{l}\right) \delta \psi_{k}\right. \\
+\int_{x_{\mathrm{s}}}^{B}\left(\left(\partial_{t} \eta+\partial_{x}\left(h \partial_{x} \phi\right)+\partial_{x}\left(\beta \partial_{x} \psi\right)\right) \delta \tilde{\phi}-\left(\partial_{t} \phi+g \eta\right) \delta \tilde{\eta}\right. \\
\left.+\left(\partial_{x}\left(\alpha \partial_{x} \psi\right)+\partial_{x}\left(\beta \partial_{x} \phi\right)-\gamma \psi\right) \delta \tilde{\psi}\right) \mathrm{d} x \\
+\frac{\int_{x_{\mathrm{s}}}^{B}\left(\left(\partial_{t} \eta+\partial_{x}\left(h \partial_{x} \phi\right)+\partial_{x}\left(\beta \partial_{x} \psi\right)\right) \varphi_{1} \delta \phi_{1}-\left(\partial_{t} \phi+g \eta\right) \varphi_{1} \delta \eta_{1}\right.}{\frac{\left.+\left(\partial_{x}\left(\alpha \partial_{x} \psi\right)+\partial_{x}\left(\beta \partial_{x} \phi\right)-\gamma \psi\right) \varphi_{1} \delta \psi_{1}\right) \mathrm{d} x}{\left.-\left.\left(h \partial_{x} \phi\right)\right|_{B^{-}} \delta \phi_{1}-\left.\left(\alpha \partial_{x} \psi+\beta \partial_{x} \phi\right)\right|_{B^{-}} \delta \psi_{1}\right] \mathrm{d} t}}
\end{array}
$$

where we introduced mass and stiffness matrices $M_{k l}, S_{k l}, A_{k l}, B_{k l}, G_{k l}$, and used endpoint conditions $\delta \eta_{k}(0)=\delta \eta_{k}(T)=0$, connection conditions $\delta \tilde{\eta}(B, t)=$ $\delta \tilde{\phi}(B, t)=\delta \tilde{\psi}(B, t)=0$, and no-normal through flow conditions at $x=x_{\mathrm{s}}, L$. The matrices in 2.12 are defined as follows

$$
\begin{aligned}
M_{k l} & =\int_{B}^{L} \varphi_{k} \varphi_{l} \mathrm{~d} x, \quad S_{k l}=\int_{B}^{L} h \partial_{x} \varphi_{k} \partial_{x} \varphi_{l} \mathrm{~d} x \quad A_{k l}=\int_{B}^{L} \alpha \partial_{x} \varphi_{k} \partial_{x} \varphi_{l} \mathrm{~d} x \\
B_{k l} & =\int_{B}^{L} \beta \partial_{x} \varphi_{k} \partial_{x} \varphi_{l} \mathrm{~d} x, \quad \text { and } \quad G_{k l}=\int_{B}^{L} \gamma \varphi_{k} \varphi_{l} \mathrm{~d} x .
\end{aligned}
$$


Provided we let the size of the zeroth element go to zero such that the underline terms in $2.12 \mathrm{~b}$ vanish, the equations arising from (2.12) are

$$
\begin{aligned}
M_{k l} \dot{\eta}_{l}-S_{k l} \phi_{l}-\underline{B_{k l} \psi_{l}}-\left.\delta_{k 1}\left(h \partial_{x} \phi+\underline{\beta \partial_{x} \psi}\right)\right|_{B^{-}} & =0 \\
M_{k l} \dot{\phi}_{k}+g M_{k l} \eta_{k} & =0 \\
A_{k l} \psi_{l}+B_{k l} \phi_{l}+G_{k l} \psi_{l}-\left.\delta_{k 1}\left(\alpha \partial_{x} \psi+\beta \partial_{x} \phi\right)\right|_{B^{-}} & =0
\end{aligned}
$$

with Kronecker delta symbol $\delta_{k l}$, one when $k=l$ and zero otherwise, and the equations 2.9a for $x \in\left[x_{\mathrm{s}}, B\right]$. Taking this limit does not jeopardize the time step, as this zeroth element lies in the continuum region, in which the resolution is infinite.

\subsection{Effective Boundary Conditions: Shallow Water Equations}

\subsubsection{Flat Bottom Case -WKB0}

We start with the shallow-water limit of 2.12 in which the bottom is flat for $x \in\left[x_{\mathrm{s}}, L\right]$ and, effectively, $\psi=0$. It is then possible to calculate the exact solution in part of the domain $\left[x_{\mathrm{s}}, B\right]$ and specify the exact boundary condition at $x=B$ given the approximate, numerical finite element solution in $x \in[B, L]$. The numerical errors arising in the simulation area will therefore remain present. When time is not discretized, our mixed numerical and analytical approach ensures that the energy in the total domain is preserved.

The Riemann invariants of the linear shallow water equations can be found by taking the spatial derivative of the Bernoulli equation in (2.9a), such that the velocity $u=\partial_{x} \phi$ emerges, and combining it with the continuity equation. One thus obtains two uncoupled linear advection equations

$$
\partial_{t}(h u \pm c \eta) \pm c \partial_{x}(h u \pm c \eta)=0
$$

with eigenvalues $\pm c= \pm \sqrt{g h}$, and outgoing and incoming Riemann invariants. These have solutions

$$
h u+c \eta=\kappa_{+}(x-c t) \quad \text { and } \quad h u-c \eta=\kappa_{-}(x+c t) .
$$

The first equation in (2.16) requires a boundary condition at $x=x_{\mathrm{s}}$, the second one a boundary condition at $x=B$ or a symmetry argument. For a domain with a vertical wall at $x=x_{\mathrm{s}}$, symmetry arguments can be used to determine that

$$
\kappa_{+}(x-c t)=-\kappa_{-}\left(2 x_{\mathrm{s}}-x+c t\right),
$$


such that $h u=h \partial_{x} \phi=\left(\kappa_{-}(x+c t)-\kappa_{-}\left(2 x_{\mathrm{s}}-x+c t\right)\right) / 2$ is indeed zero at $x=x_{\mathrm{s}}$. From (2.14), we note that we require the mass flux $h u$ at $x=B^{-}$. We determine the incoming characteristic at $x=B$ by observing and storing

$$
\kappa_{-}(B+c t)=\left.\left(h \partial_{x} \phi-c \eta\right)\right|_{x=B^{+}}
$$

using the finite element solution at $x=B^{+}$. Note that $u=\partial_{x} \phi$ is not continuous at $x=B$ and we chose its right limit. The incoming wave is reflected at $x=x_{\mathrm{s}}$ and arrives later back at $x=B$ with a delay time $\Delta \tau=2\left(B-x_{\mathrm{s}}\right) / c$, and we thus have to store the values $\kappa_{-}(B+c \tilde{t})$ from the current time till $\Delta \tau$ earlier, i.e., $\tilde{t} \in[t-\Delta \tau, t]$. Hence, we can specify the flux required in (2.14) as:

$$
\left.\left(h \partial_{x} \phi\right)\right|_{x=B^{-}}=\left(\kappa_{-}(B+c t)-\kappa_{-}\left(2 x_{\mathrm{s}}-B+c t\right)\right) / 2
$$

using the stored values from (2.18).

From (2.16) and $\partial_{t} \phi=-g \eta$ in 2.9a), it follows that the free surface deviation and the velocity potential satisfy

$$
\eta=\frac{1}{2 c}\left(\kappa_{+}(x-c t)-\kappa_{-}(x+c t)\right) \quad \text { and } \quad \phi=F_{+}(x-c t)+F_{-}(x+c t)
$$

with

$$
F_{+}^{\prime}=\frac{g}{2 c^{2}} \kappa_{+}(x-c t) \quad \text { and } \quad F_{-}^{\prime}=\frac{g}{2 c^{2}} \kappa_{-}(x+c t) .
$$

Using the above expressions, we note that the influx and observation operators $\mathcal{O}$ and $\mathcal{I}$ are given by

$$
\begin{aligned}
& \mathcal{O}(\phi)=\partial_{t} \phi+c \partial_{x} \phi=-2 g \eta_{i n c} \quad \text { and } \\
& \mathcal{I}(\phi)=\partial_{t} \phi-c \partial_{x} \phi=-2 g \eta_{\text {refl }}
\end{aligned}
$$

with $\eta_{i n c}=-\kappa_{-}(x+c t) /(2 c)$ and $\eta_{r e f l}=\kappa_{+}(x-c t) /(2 c)$. Hence, we can rewrite 2.19 as

$$
\left.\left(h \partial_{x} \phi\right)\right|_{x=B^{-}}=-\left.c\left(\eta-2 \eta_{r e f l}\right)\right|_{x=B^{-}} .
$$

Since $\eta$ by construction is continuous in $x=B$, one can also use the finite element solution $\eta_{1}$ for $\eta$ in (2.23) instead. In the numerical validation, we denote this approach by WKB0. Note that it was our goal to determine $\left.h \partial_{x} \phi\right|_{x=B^{-}}$for use in the finite element model (2.14). We achieved this goal in $(2.19)$ or 2.23$)$ using the $\kappa_{-}(\cdot)-$ function defined in (2.18).

We remark the following on the implementation. Consider, say, that we start with a quiescent state in the region $x \in\left[x_{\mathrm{s}}, B\right]$ at the initial time. At a given time we need to know $\kappa_{-}(q)$ for $q \in\left[B+c t, 2 x_{\mathrm{s}}-B+c t\right]$, or for $N_{B}+1$ values when using a fixed, discrete time step $\Delta t=2\left(B-x_{\mathrm{s}}\right) /\left(c N_{B}\right)$ in a specific time step integrator. This time step $\Delta t$ needs to be sufficiently small to ensure stability 
of the finite element solution. Yet such a fixed time step allows us to store only $N_{B}+1$ current and past values of $\kappa_{-}\left(B+c n_{t} \Delta t\right)$ with integer index $n_{t}$ in a fixed length array, which oldest stored value of $\kappa_{-}$is replaced with the current value after every time step. This can be done cyclically. Alternatively, for a variable length time integrator one would need to interpolate between the stored values of $\kappa_{-}$. For a multi-step time integrator it is advised to store also values of $\kappa_{-}(B+c t)$ at intermediate times used in the time step integration.

\subsubsection{WKB approximation -WKB1 \& WKB2}

When the bottom topography is slowly varying, it is possible to solve the equations in the shallow part $x \in\left[x_{\mathrm{s}}, B\right]$ of the domain asymptotically, using the Wentzel-Kramer-Brillouin (WKB) approximation Bender and Orszag 1978, Bremmer [1951], van Groesen and Andonowati 2011], van Groesen and Molenaar [2007], Hinch [1991], Whitham [1997]. Consider the in-situ phase speed to be a slowly varying function of space: $c=\sqrt{h(\epsilon x)}=c(\epsilon x)$. Consequently, $d c / d x=\epsilon c^{\prime}$ (with $c^{\prime}=d c / d(\epsilon x)$ ) scales as $\mathcal{O}(\epsilon)$. The variational principle for the shallow water equations, cf. (2.4), can then be rewritten by rearranging the kinetic energy term for $x \in\left[x_{\mathrm{s}}, B\right]$ as follows

$$
\begin{aligned}
0=\delta \int_{0}^{T} & {\left[M_{k l} \phi_{k} \dot{\eta}_{l}-\frac{1}{2} g M_{k l} \eta_{k} \eta_{l}-\frac{1}{2} S_{k l} \phi_{k} \phi_{l}+\right.} \\
& \left.\int_{x_{\mathrm{s}}}^{B} \phi \partial_{t} \eta-\frac{1}{2} g \eta^{2}-\frac{1}{2 g} c\left(\partial_{x}(\sqrt{c} \phi)^{2}-\frac{\epsilon c^{\prime}}{\sqrt{c}} \phi \partial_{x}(\sqrt{c} \phi)+\frac{\epsilon^{2}}{4 c} \phi^{2} c^{\prime 2}\right) \mathrm{d} x\right] \mathrm{d} t .
\end{aligned}
$$

Variation of (2.24) yields the finite element discretization (2.14) (momentarily for $\psi=0$ ) with the same mass flux as coupling term, and the equations

$$
\partial_{t} \phi+g \eta=0 \quad \text { and } \quad g \partial_{t} \eta+\sqrt{c} \partial_{x}\left(c \partial_{x}(\sqrt{c} \phi)\right)=\frac{\epsilon^{2}}{4}\left(c^{\prime 2}+2 c c^{\prime \prime}\right) \phi .
$$

By defining new variables $q=\sqrt{c} \phi$ and $p=\sqrt{c} g \eta$, and a new coordinate $\sigma=$ $-\int_{x}^{B} d \zeta / c(\epsilon \zeta)$ such that $\partial_{\sigma}=c \partial_{x}$, one obtains the system

$$
\partial_{t} q+p=0 \quad \text { and } \quad \partial_{t} p+\partial_{\sigma \sigma} q=-b q,
$$

in which $b=-\epsilon^{2}\left(c^{\prime 2}+2 c c^{\prime \prime}\right) / 4$ scales as $O\left(\epsilon^{2}\right)$.

The solution of this linear equation consists of the solution $p_{H}$ to the homogeneous problem plus a particular solution $p_{a}$, such that $p=p_{H}+p_{a}$. The homogeneous solution satisfies as in the flat bottom case again two linear advection equations. In the transformed variables $\left(v=\partial_{\sigma} q, p_{H}\right)$ and coordinates $(\sigma, t)$ these read

$$
\partial_{t}\left(v \pm p_{H}\right) \pm \partial_{\sigma}\left(v \pm p_{H}\right)=0 .
$$


Their solutions are

$$
\begin{aligned}
v+p_{H}=\kappa_{+}(\sigma-t) \text { and } \quad v-p_{H}=\kappa_{-}(\sigma+t) \\
\text { such that } p_{H}=\frac{1}{2}\left(\kappa_{+}(\sigma-t)-\kappa_{-}(\sigma+t)\right),
\end{aligned}
$$

and these also serve as leading order solutions of $(2.26)$ at $\mathcal{O}(1)$ in $\epsilon$. The particular solution is solved iteratively $p_{a}=p_{i}$ with as zeroth iteration $p_{0}=0$ and as first iteration $p_{1}$ satisfying

$$
\left(\partial_{t}-\partial_{\sigma}\right)\left(\partial_{t}+\partial_{\sigma}\right) p_{1}=b p_{H},
$$

as following from substitution of $p=p_{H}+p_{1}$ into 2.26) and ignoring the $b p_{1}$ term. To aid finding the solution, we rewrite 2.29 by introducing a new intermediate variable $r_{1}$ as

$$
\left(\partial_{t}-\partial_{\sigma}\right) r_{1}=b p_{H} \quad \text { and } \quad\left(\partial_{t}+\partial_{\sigma}\right) p_{1}=r_{1} .
$$

Given the solution $p_{H}$ in (2.28), the solution for $r_{1}$ is

$$
r_{1}=\frac{1}{2} \kappa_{-}(\sigma+t) \int_{0}^{\sigma} b(\epsilon \zeta) \mathrm{d} \zeta-\int_{0}^{\sigma} \frac{1}{2} \kappa_{+}(2 \beta-(\sigma+t)) b(\epsilon \beta) \mathrm{d} \beta .
$$

Hence, the solution for $p_{1}$ in 2.29 becomes

$$
\begin{aligned}
p_{1}=G_{2}(\sigma-t) & +\int_{0}^{\sigma} \frac{1}{2} \kappa_{-}(2 \beta-(\sigma-t)) \int_{0}^{\beta} b(\epsilon \zeta) \mathrm{d} \zeta \mathrm{d} \beta \\
& -\int_{0}^{\sigma} \int_{0}^{\gamma} \frac{1}{2} \kappa_{+}(2 \beta-(2 \gamma-(\sigma-t))) b(\epsilon \beta) \mathrm{d} \beta \mathrm{d} \gamma
\end{aligned}
$$

in which the functions $G_{2}$ is determined by the initial condition $p_{1}(\sigma, 0)=0$ as we have rest flow in the region $x \in\left[x_{\mathrm{s}}, B\right]$, or equivalently $\sigma \in\left[0, \sigma_{x_{\mathrm{s}}}\right]$ with $\sigma_{x_{\mathrm{s}}}=-\int_{x_{\mathrm{s}}}^{B} d \zeta / c(\epsilon \zeta)$. Hence, what remains is to determine $\kappa_{-}$and $G_{2}$ given the initial conditions, and the inflow of information at $\sigma=0$ for time $t>0$.

Firstly, consider the case with an open boundary and for simplicity a flat bottom for $x<x_{\mathrm{s}}$ such that there is no reflected wave at leading order. Hence, $\kappa_{+}=0$. We use $G_{2}$ such that $p_{1}(\sigma, 0)=0$. The total, asymptotic solution then becomes

$$
p=g \sqrt{c} \eta=-\frac{1}{2} \kappa_{-}(\sigma+t)+\int_{\sigma-t}^{\sigma} \frac{1}{2} \kappa_{-}(2 \beta-(\sigma-t)) \int_{0}^{\beta} b(\epsilon \zeta) d \zeta d \beta .
$$

It is the sum of the incoming wave in the first term, and the wave reflection due to the slowly varying topography in the second term. As in the flat bottom case, 
the incoming wave is determined as the boundary condition at $\sigma=0^{+}$from the finite element solution for the first Riemann invariant

$$
\kappa_{-}(t)=\left.\left(v-p_{H}\right)\right|_{\sigma=0^{+}}=\left.\left(c \partial_{x}(\sqrt{c} \phi)-g \sqrt{c} \eta\right)\right|_{x=B^{+}} .
$$

Assuming that $c^{\prime}(\epsilon x)=0$ or at least of $O\left(\epsilon^{2}\right)$ at $x=B$ ensures that the homogeneous solution holds locally, such that it follows from 2.28) and 2.33) that

$$
\begin{aligned}
\left.p\right|_{\sigma=0} & =\frac{1}{2}\left(\tilde{\kappa}_{+}(-t)-\kappa_{-}(t)\right) \\
& \left.=-\frac{1}{2} \kappa_{-}(t)-\int_{0}^{t} \frac{1}{2} \kappa_{-}(2 \beta+t)\right) \int_{0}^{\beta} b(\epsilon \zeta) d \zeta d \beta .
\end{aligned}
$$

This determines $\tilde{\kappa}_{+}$, and thus

$$
\begin{aligned}
\left.\left(h \partial_{x} \phi\right)\right|_{x=B^{-}} & =\frac{\sqrt{c}}{g} \partial_{\sigma} q=\frac{\sqrt{c}}{2 g}\left(\tilde{\kappa}_{+}(-t)+\kappa_{-}(t)\right) \\
& =\frac{\sqrt{c}}{2 g}\left(\kappa_{-}(t)-\int_{0}^{t} \kappa_{-}(2 \beta+t) \int_{0}^{\beta} b(\epsilon \zeta) d \zeta d \beta\right),
\end{aligned}
$$

where we used $\tilde{\kappa}_{+}$to denote that we really couple the homogeneous solution on a local plateau in the topography (of infinitesimal width) to the WKB solution. Alternatively, employing the approach with observation and influx operators and the finite element solution, we find as in 2.23 but extended with the reflection part in (2.33) that

$$
\begin{aligned}
\left.\left(h \partial_{x} \phi\right)\right|_{x=B^{-}} & =-\left.c\left(\eta-2 \eta_{\text {refl }}\right)\right|_{x=B^{-}} \\
& =-c \eta_{1}-\frac{\sqrt{c}}{g} \int_{0}^{t} \kappa_{-}(2 \beta+t) \int_{0}^{\beta} b(\epsilon \zeta) d \zeta d \beta,
\end{aligned}
$$

since $\eta=p /(g \sqrt{c})$. Also note that we have used the full $\mathcal{O}\left(\epsilon^{2}\right)$ expression here. The reflection term in 2.33 has a nice interpretation. An input $\kappa_{-}(t)=\delta\left(t-t_{0}\right)$ at $\sigma=0$, concerning the second term in 2.36), produces a reflection at that same point $\sigma=0$ given by

$$
\int_{0}^{\left(t-t_{0}\right) / 2} b(\epsilon \zeta) d \zeta
$$

which is the result of the reflection of the right propagating wave until the point $\sigma=\left(t-t_{0}\right) / 2$. In other words, the reflection is influxed back after a delay time $t-t_{0}=2 \sigma$. Hence, $\sigma$ serves as a scaled and shifted time coordinate. In the numerical validation, we denote this approach by WKB1.

Secondly, we consider the harbour case with a fixed wall at $x=x_{\mathrm{S}}$ or, equivalently, at $\sigma=\sigma_{x_{\mathrm{s}}}=-\int_{x_{\mathrm{s}}}^{B} d \zeta / c(\epsilon \zeta)$. Again, $G_{2}$ is used to satisfy the quiescent 
initial condition. Furthermore, $2 v=\kappa_{+}(\sigma-t)+\kappa_{-}(\sigma+t)$ should be zero at $\sigma=\sigma_{x_{\mathrm{s}}}$; hence $\kappa_{+}(\sigma-t)=-\kappa_{-}\left(2 \sigma_{x_{\mathrm{s}}}-(\sigma-t)\right)$. Note that we did not include the higher order correction of the solution in the determination of the primary reflected wave $\kappa_{-}$, which introduces a small error. The total, asymptotic solution thus becomes

$$
\begin{gathered}
p=-\left(\kappa_{-}\left(2 \sigma_{x_{\mathrm{s}}}-(\sigma-t)\right)+\kappa_{-}(\sigma+t)\right) / 2+\int_{\sigma-t}^{\sigma} \frac{1}{2} \kappa_{-}(2 \beta-(\sigma-t)) \int_{0}^{\beta} b(\epsilon \zeta) \mathrm{d} \zeta \mathrm{d} \beta \\
-\underbrace{\sigma}_{\sigma-t} \int_{0}^{\gamma} \frac{1}{2} \kappa_{+}(2 \beta-(2 \gamma-(\sigma-t))) b(\epsilon \beta) \mathrm{d} \beta \mathrm{d} \gamma
\end{gathered}
$$

The first term on the right hand side is of order one, the second term of $\mathcal{O}\left(\epsilon^{2}\right)$ and the last, underlined term is due to the reflection of the reflected incoming signal from the vertical wall. Its size depends on the total change in depth and thus the total reflection of the incoming signal, as estimated in the second term, from $x=B$ to the vertical wall at $x=x_{\mathrm{s}}$. Per case considered, one has to assess whether the underlined term in 2.39) can be neglected because it is of $\mathcal{O}\left(\epsilon^{2}\right)$, or not. In case we assume that $c^{\prime}(\epsilon x)=0$ at $x=B$, we can neglect this term. Following the same reasoning as in the previous paragraph for the open boundary, we find the mass flux

$$
\begin{aligned}
\left.\left(h \partial_{x} \phi\right)\right|_{x=B^{-}} & =-\left.c\left(\eta-2 \eta_{r e f l}\right)\right|_{x=B^{-}} \\
& =-c \eta_{1}-\frac{\sqrt{c}}{g}\left(\kappa_{-}\left(2 \sigma_{x_{\mathrm{s}}}+t\right)+\int_{0}^{t} \kappa_{-}(2 \beta+t) \int_{0}^{\beta} b(\epsilon \zeta) \mathrm{d} \zeta \mathrm{d} \beta\right) .
\end{aligned}
$$

In the numerical validation, we denote this approach by WKB2. Note that it was our goal to determine $\left.h \partial_{x} \phi\right|_{x=B^{-}}$for use in the finite element model (2.14). We achieved this goal for the EBC over slowly varying topography in 2.36) or 2.37) and (2.40) using the $\kappa_{-}(\cdot)$-function defined in 2.18).

\subsection{Modelling Effective Boundary Conditions: Bous- sinesq Equations -DWKB0, DWKB1 \& DWKB2}

In this section, we largely follow the same procedure as in the previous section, but now add dispersive effects to the wave motion. Dispersion implies that the propagation speed of a wave increases with wavelength. Dispersion cannot be neglected when we are interested to follow traveling waves from the deep ocean to the shallow areas near the coast. This is evident for relatively short waves like wind waves, but even for long tsunami waves dispersion will eventually deform the initial wave shape. It leads to low-amplitude tails of shorter waves behind the 
main wave with its long-wave components. Leading order dispersion effects are included via the linear variational Boussinesq model derived for the continuous case in (2.4) and (2.9a), and for the semi-discrete case in (2.12) and (2.14). Hence, the underlined terms are now included.

Relative to the analysis for the LSWE, we made the following changes: (i) The observation and influx operator are updated to include dispersive effects approximately for long waves. Hence, the incoming wave $\eta_{\text {inc }}$ can be determined. (ii) The group velocity is used to weigh the reflected wave signal $\eta_{r e f l}$ following an approach by Lie et al. 2014]. (iii) Given $\eta_{\text {inc }}$, we determine $\eta_{r e f l}$ using the flat-bottom or WKB approaches in shallow water with one change: we use the phase speed $c_{p}$ of the peak wave instead of $c=\sqrt{g h}$.

\subsubsection{Observation and influx operators}

Firstly, we extend the operators to include some effects of the dispersion in the LVBM. The LSWE observation operator 2.22 led to the transparent or effective boundary condition (2.23), 2.36) or (2.40) for the LSWE. Since an exact transparent-influx effective boundary condition (EBC) for the LVBM is unknown, we use an approximation for the LVBM observation operator as follows

$$
\mathcal{O}_{V}(\phi, \psi) \equiv\left(\partial_{t} \phi+c \partial_{x} \phi+c \frac{\beta}{2 h} \partial_{x} \psi\right)
$$

The first two terms are the same as for the LSWE. The third term with the additional function $\psi$ gives improvement for dispersive waves. It is derived by considering a harmonic analysis for the LVBM in the case where $\alpha, \beta, \gamma$ and $h$ are constants. By combining the two dynamic equations after substitution of $\phi, \psi, \eta \propto e^{i k x+i \omega t}$ with imaginary number $i^{2}=-1$, wave number $k$, and frequency $\omega$; one finds that $(\omega+c k) \phi-g \beta k^{2} \psi /(\omega-c k)=0$. When we focus on left propagating long waves with $\omega \approx-c k$, this relationship becomes $(\omega+c k) \phi+$ $g \beta k \psi /(2 c)=0$, which then leads to the approximate observation operator (2.41). A spectrum of the initial condition used for later simulation as in Fig. 2.2]justifies this focus on long waves.

Motivated by the effective boundary treatment for the LSWE in (2.22), we define LVBM observation and influx operators to measure the elevation of waves and impose the reflected wave signal back into the modeled part of the domain as

$$
\begin{aligned}
& \mathcal{O}_{V}(\phi, \psi)=-2 g \eta_{i n c}(t) \quad \text { and } \\
& \mathcal{I}_{V}(\phi, \psi) \equiv\left(\partial_{t} \phi-c \partial_{x} \phi-c \frac{\beta}{2 h} \partial_{x} \psi\right)=-2 g \eta_{\text {refl }}(t) .
\end{aligned}
$$




\subsection{Modelling Effective Boundary Conditions: Boussinesq Equations -DWKB0, DWKB1 \& DWKB2

From a rearrangement of the influx operator $\mathcal{I}_{V}$, we derive that

$$
h \partial_{x} \phi=\frac{c^{2}}{g} \partial_{x} \phi=-\left(c \eta-2 c \eta_{r e f l}+\frac{\beta}{2} \partial_{x} \psi\right) .
$$

Given $\eta_{i n c}$ using this new observation operator in combination with $\partial_{t} \phi=-g \eta$, we use the results derived for the LSWE in the cases WKB0, WKB1, or WKB2 to define $\eta_{\text {refl }}$. For the total mass flux required in 2.14) at $x=B^{-}$, we thus obtain:

$$
\left.\left(h \partial_{x} \phi+\beta \partial_{x} \psi\right)\right|_{x=B^{-}}=-\left.\left(c \eta-\frac{\beta}{2} \partial_{x} \psi\right)\right|_{x=B^{+}}+\left.2\left(c \eta_{r e f l}\right)\right|_{x=B^{-}},
$$

in which $\eta$ is continuous at $x=B$ so we can use the finite element value $\eta_{1}$, and in which we use the finite element solution to obtain $\partial_{x} \psi$ (we chose its right limit) because we have not solved it in the model area $x \in\left[x_{\mathrm{s}}, B\right]$. Likewise, we derive an effective boundary condition for the weighted velocity term in 2.14

$$
\left.\left(\alpha \partial_{x} \psi+\beta \partial_{x} \phi\right)\right|_{x=B^{-}}=-\left.\left(c \beta \eta / h-\left(\alpha-\frac{\beta^{2}}{2 h}\right) \partial_{x} \psi\right)\right|_{x=B^{+}}+\left.2\left(c \beta \eta_{r e f l} / h\right)\right|_{x=B^{-}} .
$$

The speed $c$ in the last term of (2.44) and 2.45) needs to be adjusted to account the dispersive properties of model. Following Lie et al. [2014], we define a function $f(t)$ to influx $s(t)=2 \eta_{\text {refl }}$ at $x=B^{-}$in both (2.44) and (2.45) in the following way. Using the point generation case, the function $f(t)$ is the inverse Fourier transform of

$$
\check{f}(\omega)=V_{g}\left(K_{1}(\omega)\right) \check{s}(\omega),
$$

where $\check{s}(\omega)$ is the Fourier transform of the desired signal $s(t)$ using the convention

$$
s(t)=\int \check{s}(\omega) e^{-i \omega t} d \omega \quad \text { and } \quad \check{s}(\omega)=\frac{1}{2 \pi} \int s(t) e^{i \omega t} d t .
$$

The group velocity is defined by $V_{g}=d \omega / d k$ and $K_{1}$ is the inverse of dispersion function $\Omega$ :

$$
\omega=\Omega(k) \Leftrightarrow k=K_{1}(\omega)
$$

For monochromatic waves, we obtain the expression

$$
f(t)=2 c_{g} \eta_{r e f l},
$$

where $c_{g}$ is the group velocity of the related wavenumber $k$. The numerical solutions of LVBM show that a weighing with the group velocity yields better results as plotted in Fig. 2.3. We generate a monochromatic wave at $x=0 \mathrm{~km}$, period 100s, above $1000 \mathrm{~m}$ depth, and ramp up the amplitude till it is $1 \mathrm{~m}$ within 


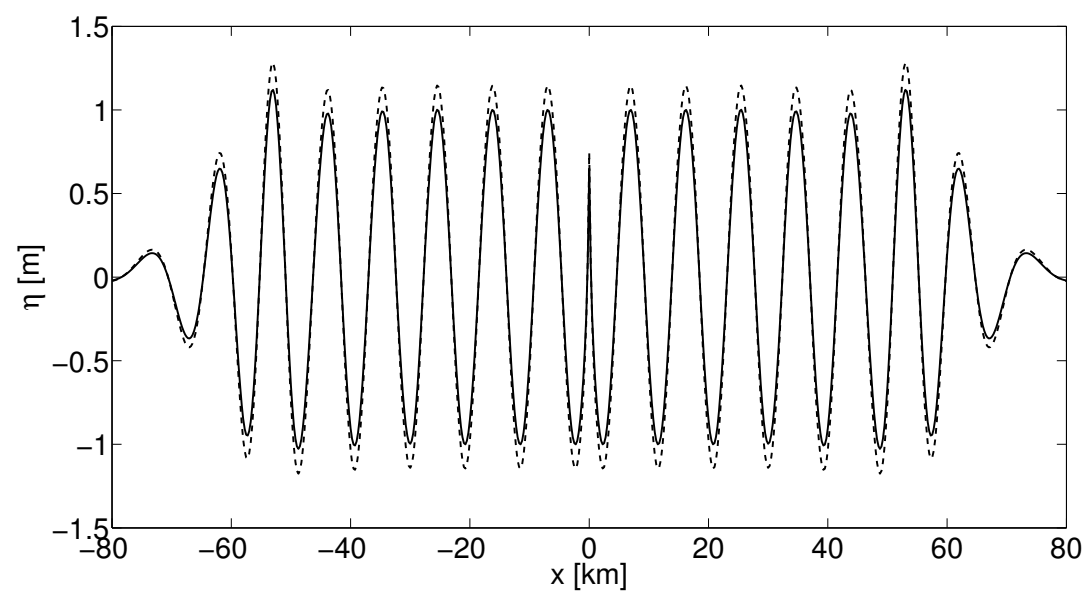

Figure 2.3: A monochromatic wave of amplitude $1 \mathrm{~m}$ is generated at $x=0 \mathrm{~km}$ with phase speed $c_{p}$ (dashed line) and group velocity $V_{g r}$ (solid line) as the weighing factor.

the first $t=200 \mathrm{~s}$. The result of using group velocity $c_{g}$ as the weighing factor is displayed by the solid line, which gives a proper amplitude of $1 \mathrm{~m}$, while the result with the dashed line uses phase velocity $c_{p}$. This finding is in accordance with the result of Lee et al. 2001 and Kim et al. 2007). For polychromatic waves, the group velocity of each corresponding frequency must be multiplied appropriately in the Fourier space as in (2.46). In the shallow water approximation, the phase speed and group velocity coincide and we therefore directly get the result as in (2.23).

Finally, since the LVBM is a dispersive model, where waves with different wavelength will travel at different phase speed, instead of $c=\sqrt{g h(x)}$ in LSWE, we take

$$
c(x)=C\left(k_{p}(x), h(x)\right)
$$

in the model area, in which $C(k, h)$ is the phase speed for wave number $k$ at depth $h$, and $k_{p}(x)$ is the wave number of the wave with the peak frequency $\omega_{p}$ at depth $h(x)$, so $\Omega\left(k_{p}(x), h(x)\right)=\omega_{p}$ for all $x$. The dispersion relation $\Omega$ and the associated phase speed $C$ can also be the exact expression for potential flow water waves, or any approximation such as the LVBM used presently. This update is used when we relate $\eta_{r e f l}$ to $\eta_{i n c}$ using the relevant expressions from WKB0, WKB1, or WKB2 for the flat bottom, slowly varying bottom case with an open or closed right boundary, respectively. These dispersive counterparts will be denoted by DWKB0 (flat bottom), DWKB1 (open right boundary) and DWKB2 (closed right boundary). 


\subsection{Numerical Validation}

A series of validation examples of increasing complexity will be considered. Firstly, a verification of the effective boundary condition is done for the LSWE in a domain with a flat bottom. These are then compared with verification of advanced effective boundary treatment for the LVBM. Secondly, we validate the effective boundary condition in a domain with a slowly varying bottom and an open flat bottom domain beyond $x=x_{\mathrm{s}}$. These results are compared directly with simulations using the advanced effective boundary treatment for the LSWE and LVBM. Finally, we analyze simulations of LSWE and the LVBM of periodic waves with a vertical wall at its end. In all cases we compare simulations in the restricted domain $x \in[B, L]$ using the effective boundary condition with simulations in the entire domain $x \in\left[x_{\mathrm{s}}, L\right]$. For the time integration, we use the fourth order ode45 solver in MATLAB. Double resolution simulations have been used throughout to double-check our simulations. We also implemented the second-order modified midpoint rule to independently check the time integration. The modified midpoint integrator allowed us to efficiently store the history integrals in the EBCformulation, while using a fixed time step that was an integer of the relevant travel time, see Section 2.3.1. Matlab's ode45 solver was, however, more efficient. Since this ode45 solver uses its own time step, we employ linear interpolation to get the correct incoming or reflected signal at the desired, fixed time step.

\subsubsection{Flat Bottom Situation}

A straightforward one-dimensional example is given by waves over a flat bottom, with depth $h_{0}$, which are reflected at $x=x_{\mathrm{s}}$. The initial "N-wave" profile taken is

$$
\eta(x, 0)=A f(x) / S \text { with } f(x)=\frac{\mathrm{d}}{\mathrm{d} x} \exp \left(-\left(x-x_{0}\right)^{2} / w_{0}^{2}\right) \text { and } S=\max (f(x))
$$

and the initial velocity potential is zero. We take $A=1 \mathrm{~m}$, the position of the wave profile $x_{0}=0.6 \mathrm{~km}$, width $w_{0}=40 \mathrm{~m}$, and constant depth $h_{0}=10 \mathrm{~m}$. We consider a full domain with $L=1.2 \mathrm{~km}$ and a restricted one with $B=0.2 \mathrm{~km}$. Spatial and temporal steps are $\Delta x=0.5 \mathrm{~m}$ and $\Delta t=0.1 \mathrm{~s}$, and we simulate till $t=120 \mathrm{~s}$.

In Fig. 2.4, we compare simulations in the entire domain $x \in\left[x_{\mathrm{s}}, L\right]$ with the ones using the EBC, for the LSWE. We used a fully reflecting wall at $x=x_{\mathrm{S}}$ and a transparent boundary condition at $x=L$ (using the flat bottom WKB0 technique). The dashed line represents the reflected wave calculated in the whole domain and the solid one represents the reflected wave using EBC. A good agreement between the two simulations is observed, as expected. 
(a)

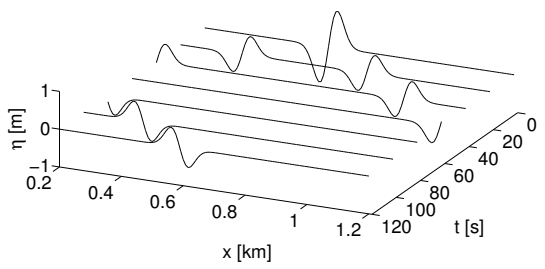

(b)

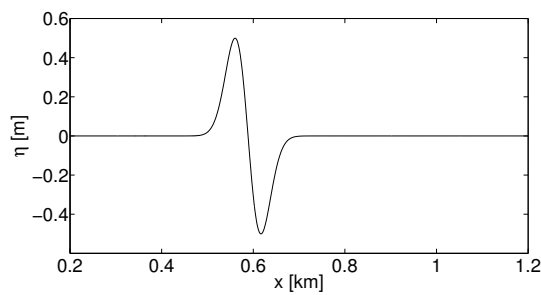

Figure 2.4: We compare numerical results between a simulation in the whole domain (dashed line) and one with the $E B C$ (solid line) for the $L S W E$ with $B=0.2 \mathrm{~km}$. The dashed and solid lines are on top of one another. Wave profiles at times $t=$ $0,20,40, \ldots, 120$ s are shown in (a) for both simulations, and in (b) a snapshot is given at $t=120 \mathrm{~s}$.

(a)

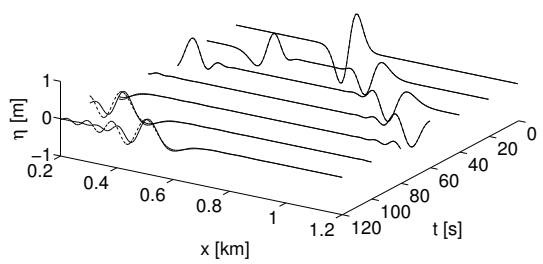

(b)

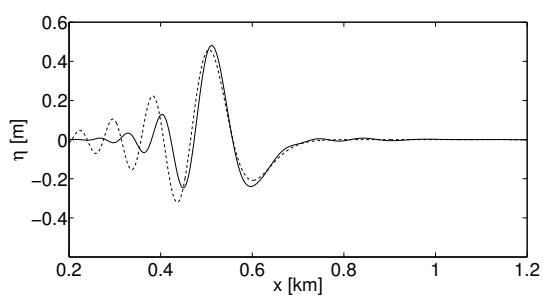

Figure 2.5: As Fig. 4 for simulations using the LVBM. The deviations are caused by the approximation to the dispersive wave propagation in the model area $\left[x_{\mathrm{s}}, B\right]=[0,0.2] \mathrm{km}$. Full LVBM: dashed line; LVBM with DWKB0: solid line.

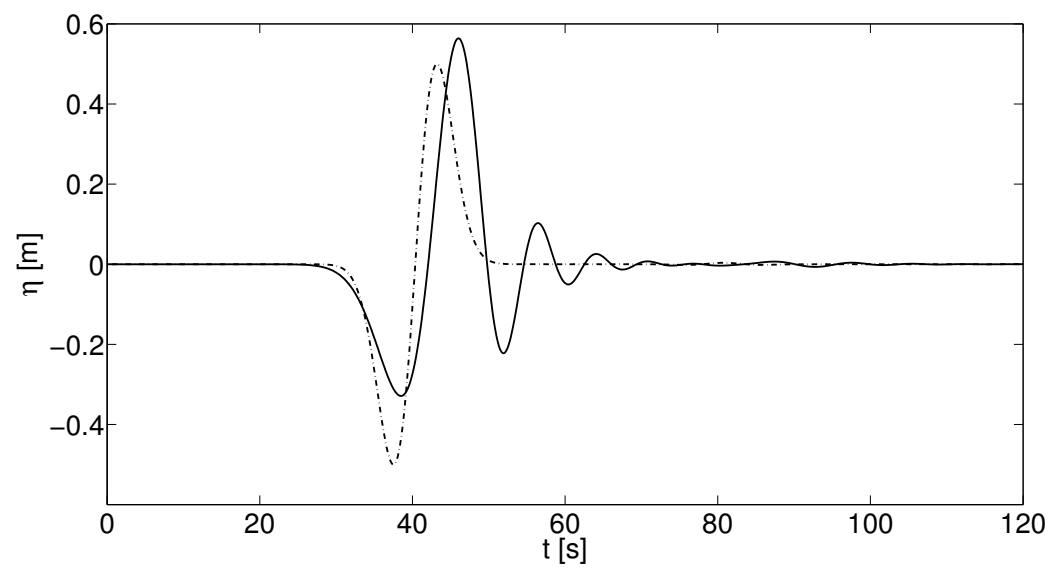

Figure 2.6: Comparison of the calculated signal at $x=B$ between LSWE (dotted-dashed line) and LVBM (solid line). 


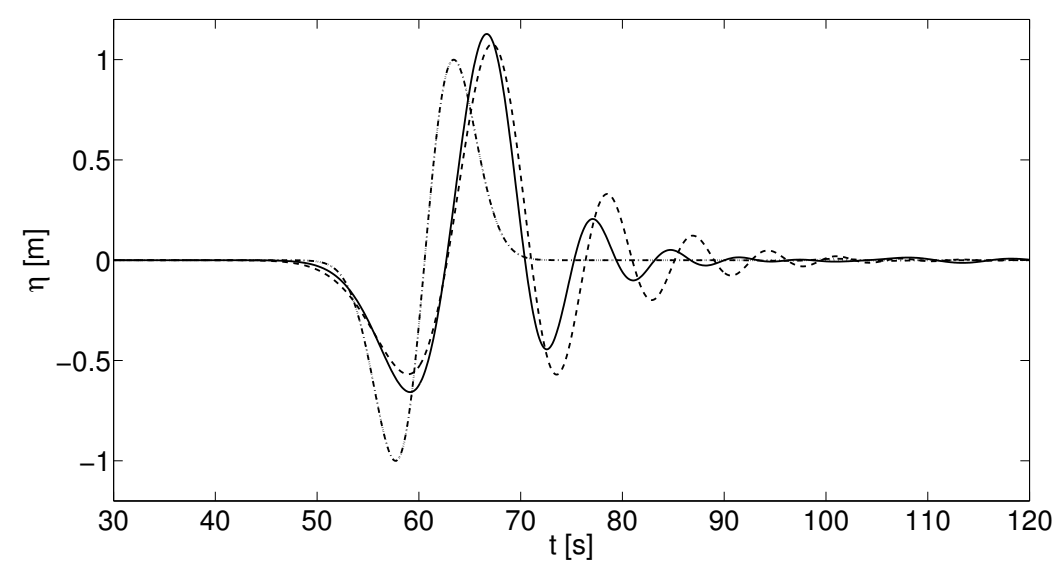

Figure 2.7: Comparison of calculated signals at $x=x_{\mathrm{s}}$ between LSWE-WKBO (dotteddashed line) and LVBM-DWKBO (solid line), together with measured signal at $x=x_{\mathrm{s}}$ in full domain simulation with LSWE (dotted line - coinciding with the dotted-dashed line) and LVBM (dashed line) for flat bottom situation.

Next, we compare simulation results using the LVBM in a domain with a flat bottom. In Fig. 2.5, we show reflected waves for simulations in the entire domain and ones with the EBC in part of the domain. Again we have a hardwall boundary condition at $x=x_{\mathrm{s}}$ and a transparent one at $x=L$ (using the DWKB0 technique, the flat bottom WKB0 extension to the LVBM). An extension of the domain for $x>L$ was made to avoid unnecessary reflection from the left transparent boundary. The dashed line represents the reflected wave calculated in the whole domain, and the solid line represents the reflection wave using the EBC. At $t=80 \mathrm{~s}$, when the reflected wave enters the simulation area again, we can see that the first peak wave travels with the same speed in both simulations, while the rest have some differences in speed and amplitude. This is expected since we use a partially non-dispersive analytical model in the model area. A plot of the scaled spectrum of the initial condition was already shown in Fig. 2.2. The simulation using the EBC gives a faster and higher wave since the reflection model only uses the phase speed of the most dominant wave in the spectrum, while the shorter waves actually should have travelled with a slower speed. The deviations decrease when the length of the model area is smaller.

In Fig. 2.6, the measured wave elevations at $x=B$ for LSWE and LVBM simulations in the whole domain are shown. In the LSWE simulation, the wave profile will remain the same as its initial form, while in LVBM the wave has been dispersed. The higher wave amplitude and successive waves in the LVBM simulation show the importance of using a dispersive model for tsunami simula- 
(a)

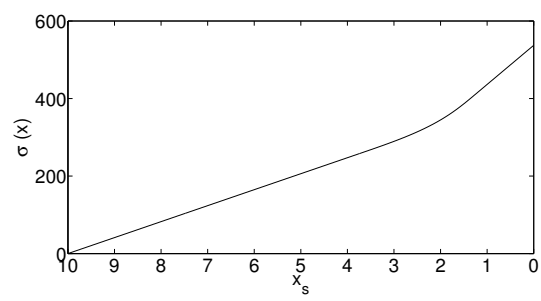

(b)

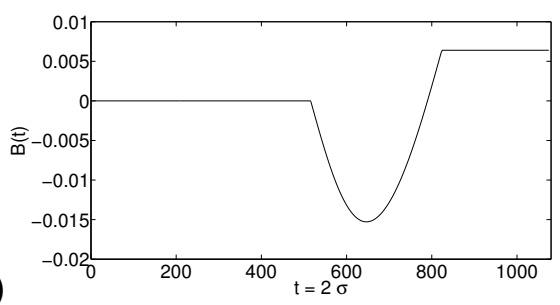

Figure 2.8: (a) A plot of the travel time from $B=10 \mathrm{~km}$ and (b) the transfer function $\overline{\mathcal{B}}$ against $\left(t-t_{0}\right)=2 \sigma$ for $w=1.25 \mathrm{~km}$ (slope $1: 50$ ). We chose $t_{0}=0$.

tion especially in the shallower coastal waters over the continental shelf. We also show wave elevations at $x=x_{\mathrm{s}}$ in Fig. 2.7. These signals are higher due to the hard-wall reflection.

\subsubsection{Slowly Varying Topography}

We consider three cases with an increasingly realistic varying bottom profile. Instead of dealing with the full run-up and run-down phenomena, we consider simpler cases of a slowly varying bathymetry between two constant depths and an open boundary on the left of $x=x_{\mathrm{s}}$. The first two cases deal with a long tsunamitype of wave approaching the coast: one with synthetic bathymetry and one with a bathymetry akin to the one near Aceh, Sumatra, Indonesia. The third case deals with periodic waves and the coastline is replaced by a reflecting hard wall at a certain shallow depth. For this case, we use a bathymetry that resembles the one near Cilacap harbor, Java, Indonesia. Bathymetry data of Aceh are obtained from the General Bathymetric Chart of the Oceans (GEBCO) with one minute accuracy (approximately 1830m). While bathymetry of Cilacap is obtained from a combination of GEBCO's data and a digitized map of local bathymetry from the Hydro-Oceanographic Office (www.dishidros.go.id) with $100 \mathrm{~m}$ accuracy. In all cases, we will present the results of simulations using LSWE in the simulation area - with WKB1 in the model area, and using the LVBM in the simulation area - with DWKB1 in the model area. We use an irregular grid according to the depth with ratio $\sqrt{h_{1} / h_{0}}$, as the decrease of the wavelength when traveling from a deep region with depth $h_{0}$ and a shallower region with depth $h_{1}$ in linear wave theory. 


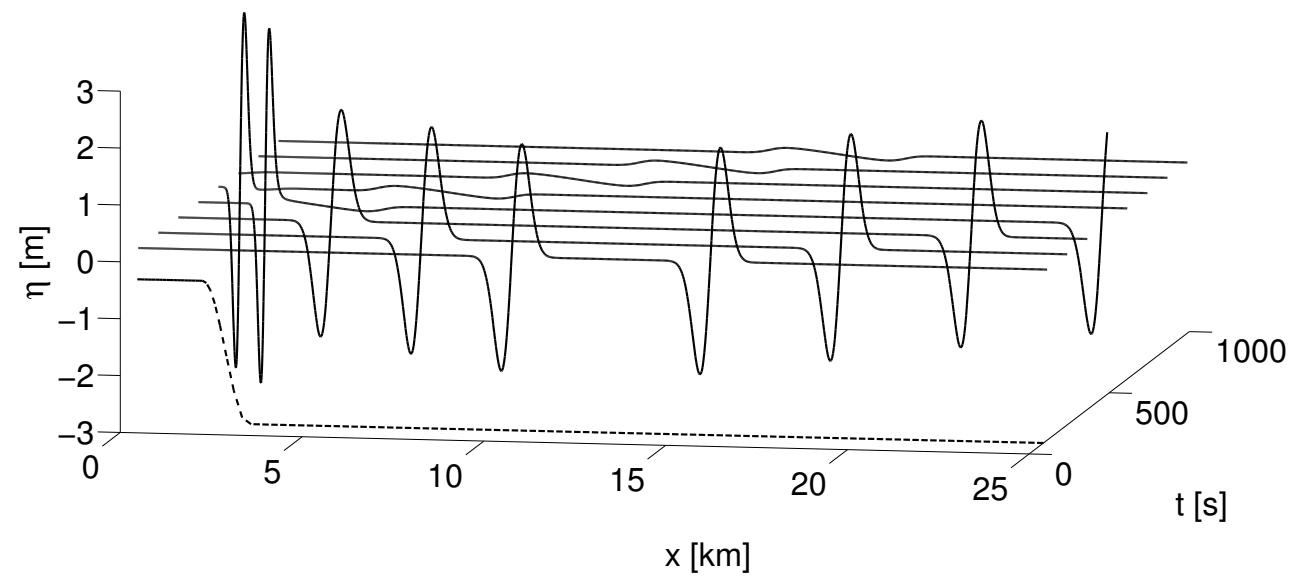

Figure 2.9: A zoom-in of a simulation in the whole domain for a steep slope (here $w=0.625 \mathrm{~km}$ ) with the LSWE. The scaled bathymetry profile is plotted with the dashed line.

\section{Simulation using synthetic bathymetry with transparent coastline}

The idealized bathymetry considered in the next example is defined by

$$
h(x)=\frac{h_{0}-h_{1}}{2} \cos \left(\pi \frac{x-m+w}{2 w}\right)+\frac{h_{0}+h_{1}}{2},
$$

for $x=[m-w, m+w]$. Parameter values used are $m=2.5 \mathrm{~km}$ as the middle of the slope and the half width of the slope $w$ is varied, $h_{0}=60 \mathrm{~m}$ is the depth in deep water, before the slow transition to a depth $h_{1}=10 \mathrm{~m}$ in shallower water. The domain is $[0,25] \mathrm{km}$ and we simulate till $t=1050 \mathrm{~s}$. We used $\Delta x=12.5 \mathrm{~m}$ at the shallowest area and $\Delta t=1.25 \mathrm{~s}$. Transparent boundary conditions have been used at both boundaries $x=x_{\mathrm{s}}, L$. An extension of the domain is made for $x>L$ to avoid the reflection from the backward traveling signal at the left transparent boundary. The initial wave profile is given by the same function (2.49), with initial amplitude $4 \mathrm{~m}$ and $x_{0}=13 \mathrm{~km}$. The width parameter of this wave profile is $w_{0}=0.4 \mathrm{~km}$. The initial velocity is zero everywhere.

For a bathymetric profile with $w=1.25 \mathrm{~km}$, a plot of the travel time for the LSWE case from $x=B=10 \mathrm{~km}$ till $x_{\mathrm{s}}=0 \mathrm{~km}$ is given in the left panel of Fig. 2.8, The right panel in Fig. 2.8 depicts the transfer function $\overline{\mathcal{B}}(\sigma)=\int_{0}^{\sigma} b(\epsilon \zeta) d \zeta$ used in (2.33) as function of the travel time $t=2 \sigma$. Hence, $\sigma$ serves as a scaled and shifted travel time. We notice that until $x=3.75 \mathrm{~km}$ the travel time increases linearly, whereafter the wave travels more slowly until $x=1.25 \mathrm{~km}$ as the depth 
(a)

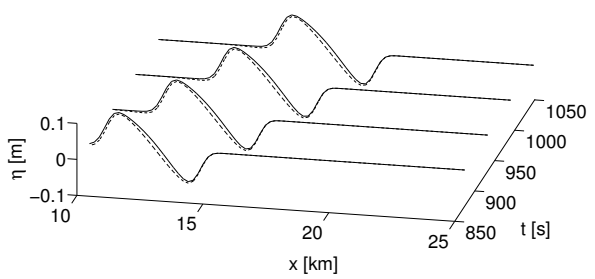

(c)

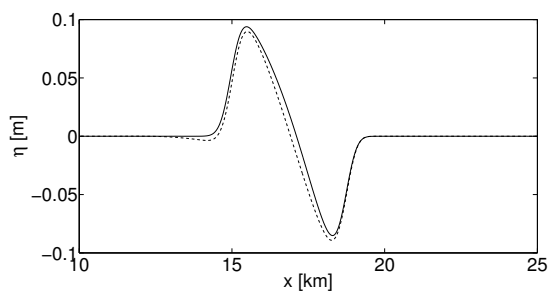

(b)

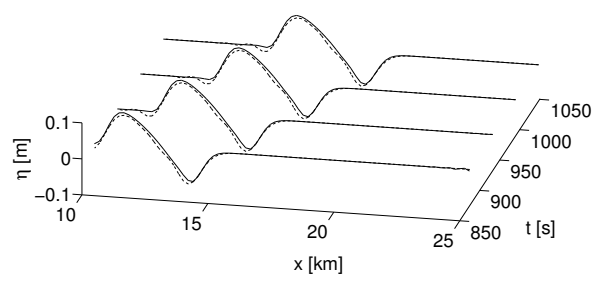

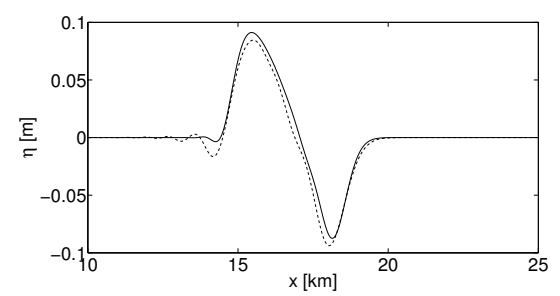

Figure 2.10: Comparison of the reflected wave between the simulation in the whole domain (dashed line) and one using the EBC (solid line) for a steep slope $(w=0.625 \mathrm{~km})$, and snapshots at $t=1050 \mathrm{~s}$. The LSWE with WKB1 results are displayed in (a), (c) and the LVBM with DWKB1 results in (b), (d).

becomes shallower. Subsequently, travel time increases linearly again since the wave continues over flat bathymetry. We therefore see that the signal arrives the escarpment at $t=515.2 \mathrm{~s}$, since we used $t_{0}=0$, and that the wave-topography interaction lasts till $t=822.4 \mathrm{~s}$.

A simulation in the whole domain with the LSWE is shown in Fig. 2.9 together with the scaled bathymetry (dashed line). A small reflection from the bathymetry can be seen at later times after the wave enters the shallows. A comparison of reflected waves between simulations in the whole domain and ones with the EBC in part of the domain is displayed in Figs. 2.102 .12 for $w=0.625 \mathrm{~km}$ (slope 1:25), $w=1.25 \mathrm{~km}$ (slope $1: 50$ ), and $w=2.5 \mathrm{~km}$ (slope 1:100), respectively. The dashed line represents the reflected wave calculated in the whole domain $[0,25] \mathrm{km}$, and the solid one represents the reflected wave using the EBC at $x=10 \mathrm{~km}$. LSWEWKB1 results are shown in a,c), while LVBM-DWKB1 results are shown in b,d). We clearly see, for both models, that there are some errors between the numerical solution in the whole domain and the one using the EBC. These errors are likely asymptotic, caused by the fact that the bathymetry does not vary slowly enough, as the analytical solution is only valid for a slowly varying bathymetry. It can be seen that when the slope is steeper, the error is larger, as expected. Both results also show that simulations with the EBC yield slightly higher wave amplitude than simulations in the whole domain.

When the LVBM with the DWKB1 EBC is used, we can also see that the 

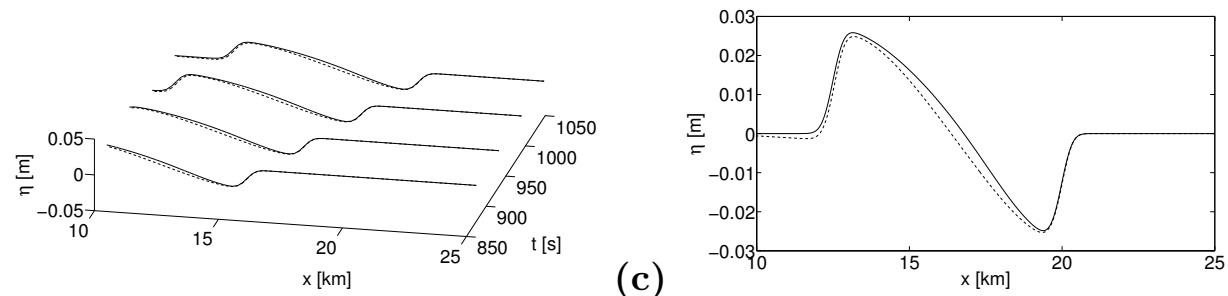

(a)

(c)

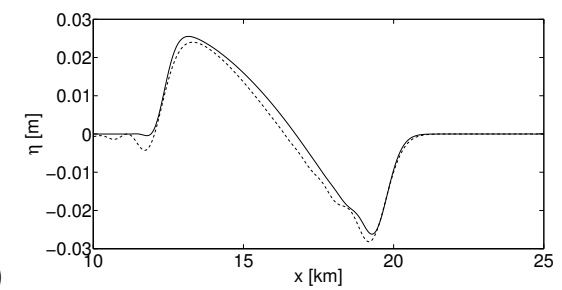

(b)

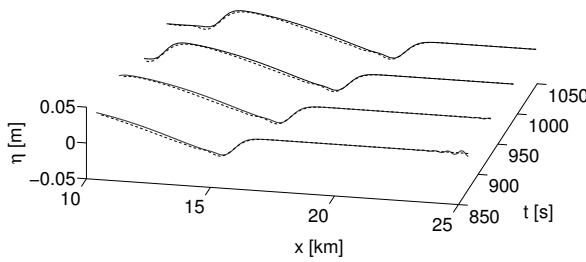

(d)

Figure 2.11: Comparison of the reflected wave between the simulation in the whole domain (dashed line) and using the $E B C$ (solid line) for a mild slope $(w=1.25 \mathrm{~km})$ and snapshots at $t=1050 \mathrm{~s}$. The LSWE with WKB1 results are displayed in (a), (c) and the LVBM with DWKB1 in (b), (d).

(a)

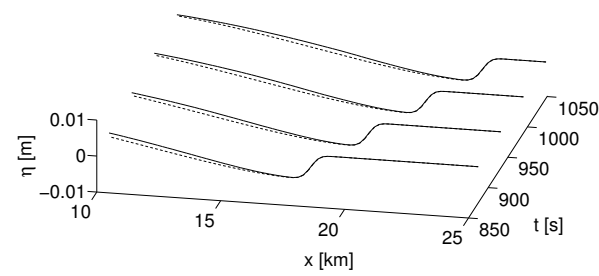

(c)

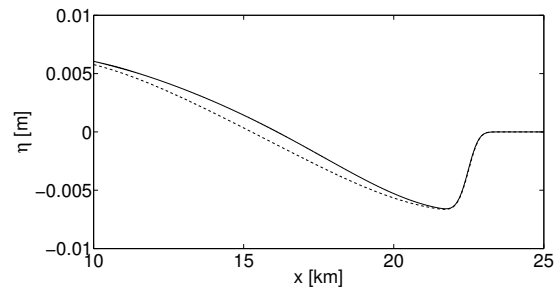

(b)

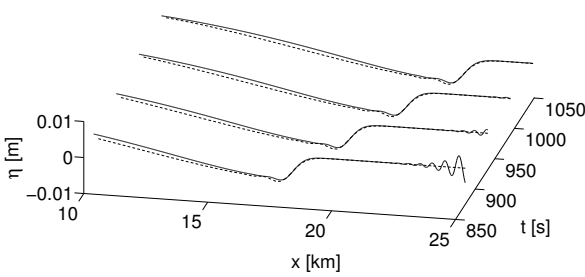

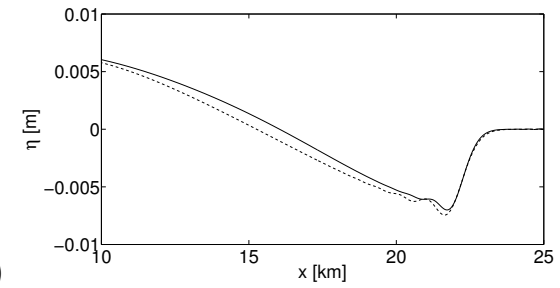

Figure 2.12: Comparison of the reflected wave between the simulation in the whole domain (dashed line) and using EBC (solid line) for very mild slope $(w=2.5 \mathrm{~km})$ and the snapshots at $t=1050 \mathrm{~s}$. The LSWE with WKB1 results are displayed in (a), (c) and the LVBM with DWKB1 results in (b), (d). 


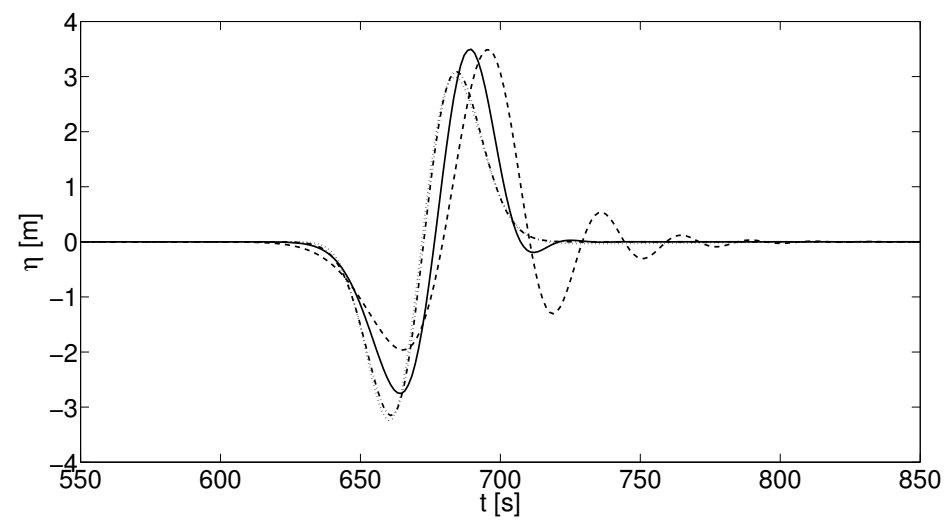

Figure 2.13: Comparison of calculated signals at $x=x_{\mathrm{s}}$ between LSWE-WKB1 (dotteddashed line) and LVBM-DWKB1 (solid line), together with measured signals at $x=x_{\mathrm{s}}$ in a full domain simulation with LSWE (dotted line) and LVBM (dashed line) for a steep slope.

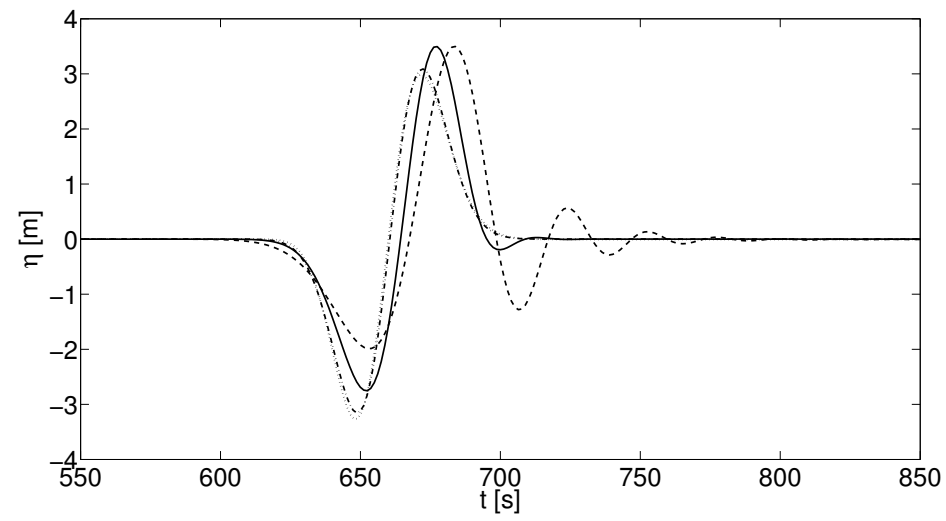

Figure 2.14: Comparison of calculated signals at $x=x_{\mathrm{s}}$ between LSWE-WKB1 (dotteddashed line) and LVBM-DWKB1 (solid line), together with measured signals at $x=x_{\mathrm{s}}$ in a full domain simulation with LSWE (dotted line) and LVBM (dashed line) for a mild slope. 


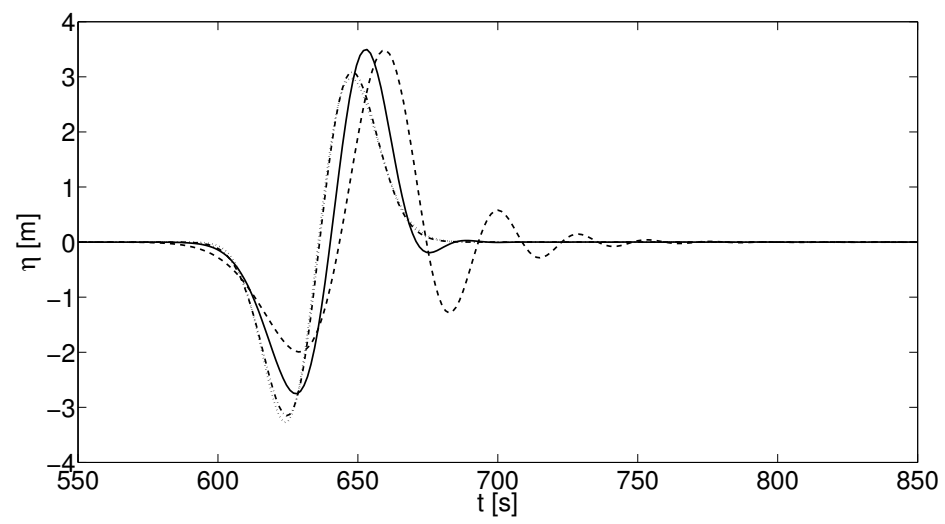

Figure 2.15: Comparison of calculated signals at $x=x_{\mathrm{s}}$ between LSWE-WKB1 (dotteddashed line) and LVBM-DWKB1 (solid line), together with measured signals at $x=x_{\mathrm{s}}$ in a full domain simulation with LSWE (dotted line) and LVBM (dashed line) for a very mild slope.

waves with EBC (solid line) are slightly faster than waves for simulations in the whole domain. That is the reflected wave has travelled a bit further towards the right. This is in agreement with the result from the previous section, since the reflection DWKB1 model only uses the phase speed of the peak wave. The $L^{2}$-errors between the numerical solutions in the whole domain and ones using the EBC are found in Table 2.1, for WKB1 and DWKB1. This error is defined as

$$
\sqrt{\int_{B}^{L}\left(\eta_{\text {whole }}-\eta_{\mathrm{EBC}}\right)^{2} \mathrm{~d} x}
$$

with $\eta_{\text {whole }}$ the finite element solution in the whole domain, and $\eta_{\mathrm{EBC}}$ the one in the simulation area only.

\begin{tabular}{|c|c|c|}
\hline slope & WKB1 $L^{2}$-error $\left[\mathrm{m}^{2}\right]$ & DWKB1 $L^{2}$-error $\left[\mathrm{m}^{2}\right]$ \\
\hline $1: 25$ & $4.61 \times 10^{-1}$ & $4.54 \times 10^{-1}$ \\
\hline $1: 50$ & $6.15 \times 10^{-2}$ & $5.68 \times 10^{-2}$ \\
\hline $1: 100$ & $7.12 \times 10^{-3}$ & $5.91 \times 10^{-3}$ \\
\hline
\end{tabular}

Table 2.1: The $L^{2}$-error between numerical solutions in the whole domain and ones using the EBC for the LSWE-WKB1 model (second column) and LVBM-DWKB1 model (third column).

The results using DWKB1 show that there are some reflections from the transparent boundary condition at $x=B$ used in the LVBM, reflections that 
(a)

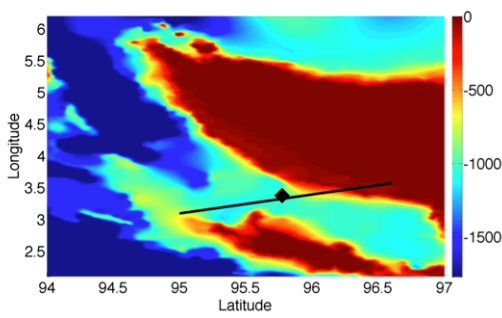

(b)

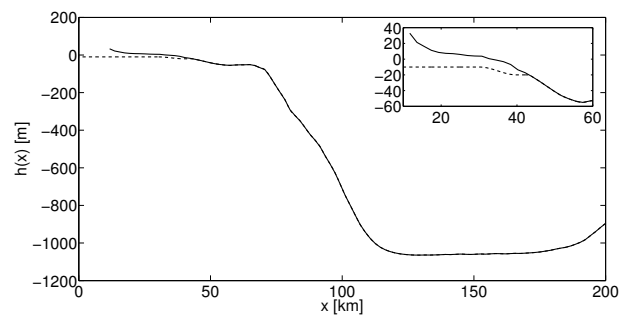

Figure 2.16: (a) Bathymetry near Aceh and (b) the cross section at $\left(95.0278^{\circ} E\right.$, $\left.3.2335^{\circ} \mathrm{N}\right)-\left(96.6583^{\circ} \mathrm{E}, 3.6959^{\circ} \mathrm{N}\right)$. The solid line concerns the bathymetry data and the dashed line concerns the approximation.

have travelled back to $x=L$ at $t \approx 900$ s. We notice these small rightward travelling waves in the simulations using the EBC (solid line) in Figs. 2.10 $2.12 \mathrm{~b}$ ), besides the larger reflection from the slowly varying bathymetry (see the very mild slope case, when the reflection from the bathymetry is smallest). This reflection arising from the transparent boundary condition appears because our transparent boundary condition (2.41) for the LVBM is only an approximation, which is optimal for long waves.

Finally, a comparison between the simulations using the EBC for both the LSWE and the LVBM in Figs. 2.13 2.15 shows that the WKB approximation is very good for the LSWE and less good for the LVBM. However, the peak amplitude is well approximated, but there is a phase shift and the EBC results show milder oscillations. The reason is that we measure in deeper water at $x=B$ and subsequently use a WKB-approach based on non-dispersive shallow water modelling, while in the full simulation dispersion becomes important in the shoaling waters.

\section{Simulation using simplified Aceh bathymetry with transparent coast- line}

Bathymetry near Aceh, Indonesia, is displayed in Fig. 2.16. The left figure concerns bathymetry data from GEBCO, with zero value for the land. The right figure concerns the cross section at $\left(95.0278^{\circ} \mathrm{E}, 3.2335^{\circ} \mathrm{N}\right)-\left(96.6583^{\circ} \mathrm{E}, 3.6959^{\circ} \mathrm{N}\right)$ shown by the solid line. The 2004 Indian Ocean tsunami was occurred with a magnitude of $\mathrm{Mw} 9.1$ at the epicenter point $95.854^{\circ} \mathrm{E}, 3.316^{\circ} \mathrm{N}$, that is shown by the symbol. In the simulation area $x \in[40,200] \mathrm{km}$, we follow the real bathymetry of Aceh. In the model area $x \in[0,40] \mathrm{km}$, we use a simplified bathymetry that is shown by the dashed line, and defined by the function 2.50 with parameter values $h_{0}=20 \mathrm{~m}, h_{1}=10 \mathrm{~m}, m=35 \mathrm{~km}, w=5 \mathrm{~km}$, continued with a constant depth of $10 \mathrm{~m}$. We will show simulations for the LSWE with WKB1 and the 


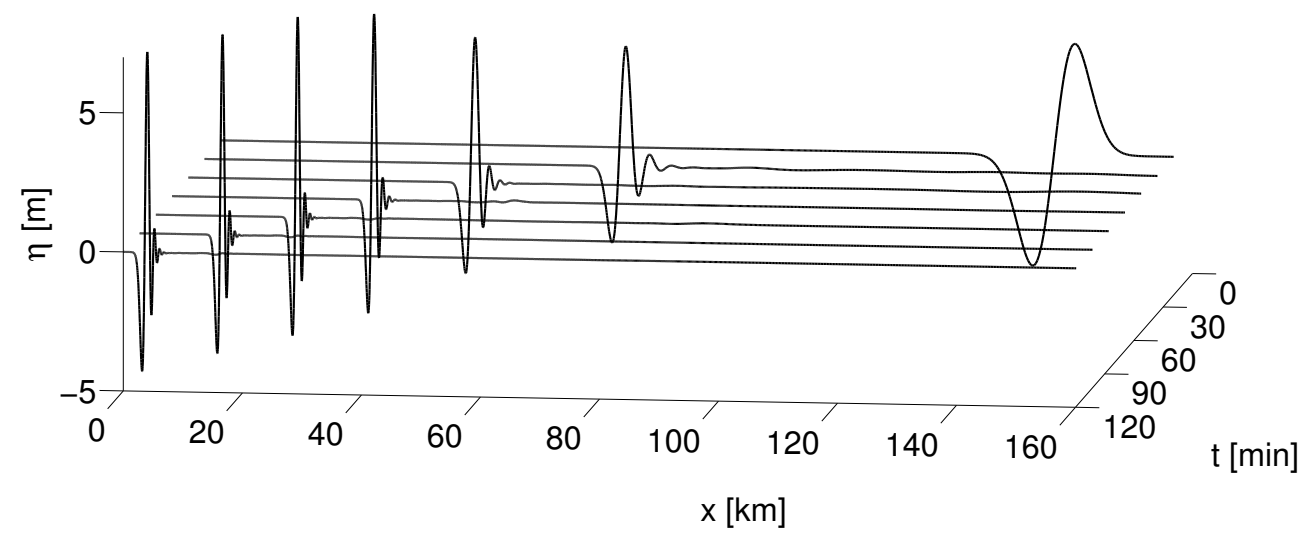

Figure 2.17: Simulation results for the Aceh case in the entire domain for the LVBM model.

(a)

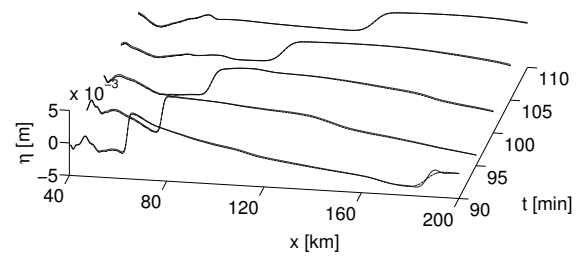

(b)

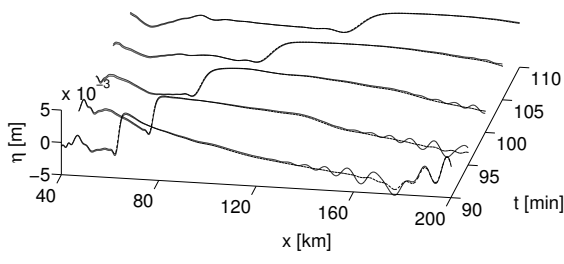

(c)

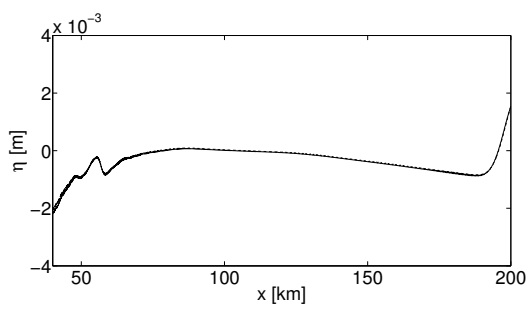

(d)

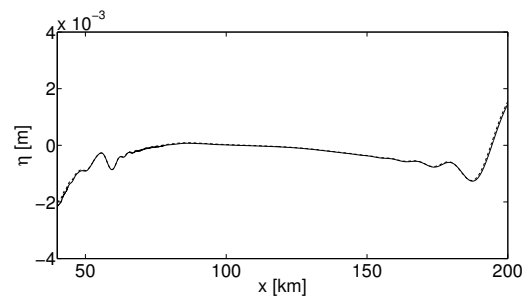

Figure 2.18: Comparison between simulation in the whole domain (dashed line) and using the EBC (solid line), and snapshots at $t=120 \mathrm{~min}$. The LSWE with WKB1 results are displayed in (a), (c) and LVBM with DWKB1 in (b), (d). 


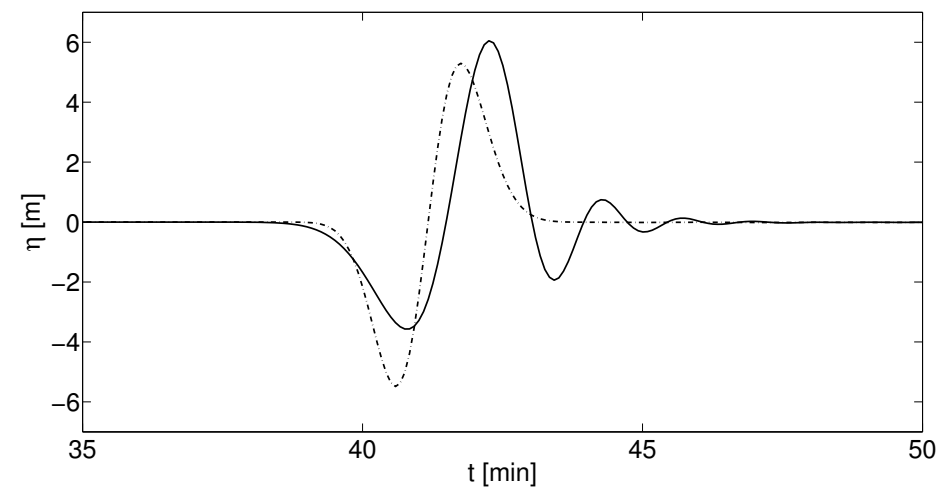

Figure 2.19: Comparison of calculated signals at $x=B$ between LSWE-WKB1 (dotteddashed line) and LVBM-DWKB1 (solid line) for the Aceh case.

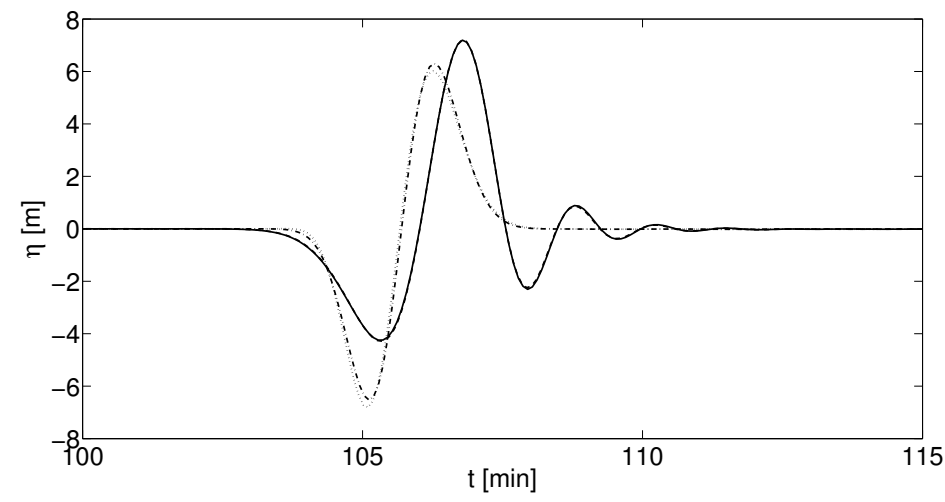

Figure 2.20: Comparison of calculated signals at $x=x_{\mathrm{s}}$ between LSWE-WKB1 (dotteddashed line) and LVBM-DWKB1 (solid line), together with measured signals at $x=x_{\mathrm{s}}$ in full domain simulation with LSWE (dotted line) and LVBM (dashed line) for the Aceh case. 
LVBM with DWKB1. The boundary at $x=L$ is transparent. The grid size used in the simulation area is $\Delta x=35 \mathrm{~m}$ at the shallowest part near $x=B$. It is reasonable for a tsunami simulation as Horillo et al. (2006) use $\Delta x=10 \mathrm{~m}$ in the onshore area for one dimensional simulations. The time step is $\Delta t=5 \mathrm{~s}$ until $t=120 \mathrm{~min}$.

The initial wave profile is again given by the profile in $(2.49)$ with zero initial velocity. The initial amplitude is $A=4 \mathrm{~m}, x_{0}=140 \mathrm{~km}$ is the position of the initial wave profile, and $w_{0}=5 \mathrm{~km}$ is its 'width'. The simulation results in the whole domain for LVBM are displayed in Fig. 2.17, in which we clearly see the break up of the initial wave profile in multiple waves tailgating the main wave in the shallow waters at $x \in[0,40] \mathrm{km}$. The comparison between the numerical solution in the whole domain (dashed line) and the simulation using the EBC within the domain (solid line) is shown in Fig. 2.18 at $120 \mathrm{~min}$. The results of the LSWE with WKB1 are shown in a,c) and the ones of LVBM with DWKB1 in $b, d)$. We can see that dispersion causes negligible differences in the reflected waves.

In Fig. 2.19, the comparisons of wave elevation measured at $B=40 \mathrm{~km}$ for LSWE with WKB1, LVBM with DWKB1, and the full simulation are shown. As in the flat bottom case, here we can see that the LVBM model gives a higher amplitude (about $1 \mathrm{~m}$ height) and a wave tail. Later, these waves will amplify as the depth gets shallower and also will disperse more as shown in Fig. 2.17. The difference clearly indicates that dispersion effects should be taken into acount in tsunami propagation. The signals arriving at $x=x_{\mathrm{s}}$ are displayed in Fig. 2.20, for LSWE with WKB1, LVBM with DWKB1, and the full simulations. It shows that the simulations with the EBC are very good, also in comparison with the previous case, presumably because we have started the model zone at $x=B$ where the water depth is shallow.

\subsubsection{Periodic waves and wall reflections}

The third case concerns periodic waves entering a harbor, where dispersion can also be important when the depth becomes more shallow. When the coastline in the harbor is modeled by a hard wall, further reflections arise. We use bathymetric data nearby Cilacap harbor, Java, Indonesia, displayed in Fig. 2.21. The left figure concerns bathymetry data from the GEBCO and the Hydro-Oceanographic Office, with zero value for the land. The right figure concerns the cross section at $\left(106.895^{\circ} \mathrm{E},-6.042^{\circ} \mathrm{N}\right)-\left(106.9533^{\circ} \mathrm{E},-6.0682^{\circ} \mathrm{N}\right)$ shown by the solid line. The simulation in the entire domain simulation concerns a domain $x \in[0,7] \mathrm{km}$ in which the real bathymetry is used in the simulation area for $x \in[1,7]$. The simplified bathymetry used in the model area is shown by the dashed line, and defined by a function (2.50) with $h_{0}=6 \mathrm{~m}, h_{1}=1 \mathrm{~m}, m=0.5 \mathrm{~km}, w=0.5 \mathrm{~km}$, for 
(a)

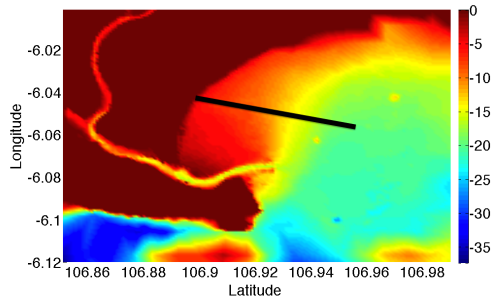

(b)

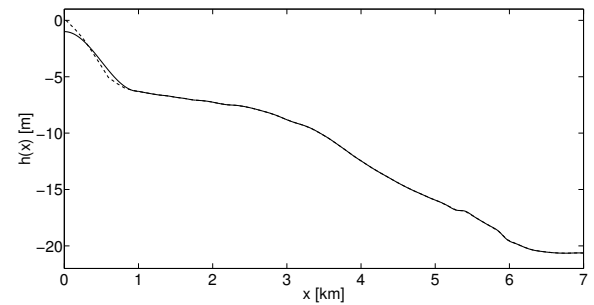

Figure 2.21: (a) Bathymetry near Cilacap and (b) the cross section at $\left(106.895^{\circ} \mathrm{E}\right.$, $\left.-6.042^{\circ} \mathrm{N}\right)-\left(106.9533^{\circ} \mathrm{E},-6.0682^{\circ} \mathrm{N}\right)$. The solid line concerns the bathymetry data and dashed line concerns the approximation.

$x \in[0,1]$. We influx a periodic wave with amplitude $0.4 \mathrm{~m}$ and wave period $25 \mathrm{~s}$. The smallest grid size in the simulation area near $x=B$ is $\Delta x=2.5 \mathrm{~m}$, and the time step is $\Delta t=1 \mathrm{~s}$ till $t=20 \mathrm{~min}$.

In Fig. 2.22, the comparison between the numerical solution in the whole domain (dashed line) and the one using the EBC (solid line) is shown. The results with the LSWE and WKB2 are shown in a,c) and the ones with the LVBM and DWKB2 are shown in b,d). We can see that at later times there are differences between the numerical solution in the whole domain and the one using EBC due to the asymptotic nature of the WKB approximation. In contrast with previous cases, where the reflections arise only for one $N$-wave, this case shows the capability of our EBC to continuously measure the incoming wave and influx the reflection during their interactions. The signals at the end of the harbor at $x=x_{\mathrm{s}}$ are shown in Fig. 2.23 and 2.24 for various simulations. It shows the strength of our approach using the EBC. In LVBM simulations, the wave arrives later at $x=x_{\mathrm{s}}$ since its wave speed is smaller than in LSWE. Finally, wave dispersion is important and the LVBM with and without EBC yields better predictions than LSWE.

\subsection{Conclusions}

We have formulated an effective boundary condition (EBC) for the nondispersive, linear, shallow water equations (LSWE) and the dispersive, linear, variational Boussinesq model (LVBM). The EBC is used as boundary condition in a domain divided into a simulation area and a model area. As the names indicate, the numerical model with the EBC is shorter and the wave reflection in the model area is (approximately) modeled analytically. The model area typically concerns the shallow bathymetry near the coast, which tends to be computationally costly in a numerical model for the entire domain. For that reason, the shallow coastal 
(a)
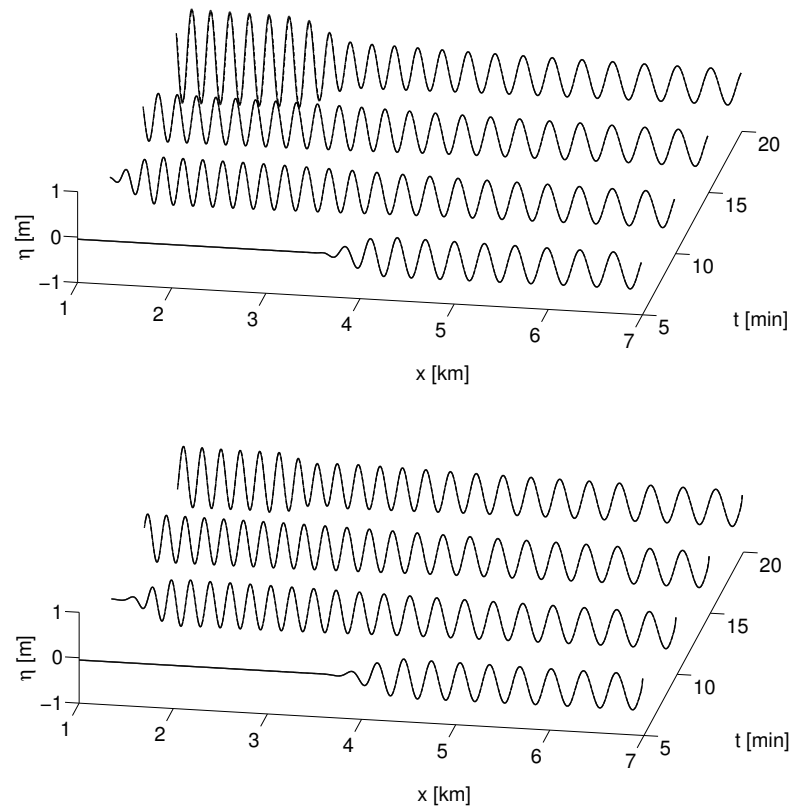

(b)

(c)

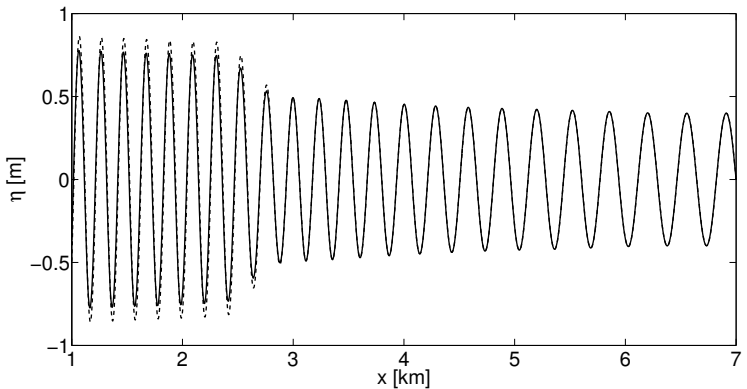

(d)

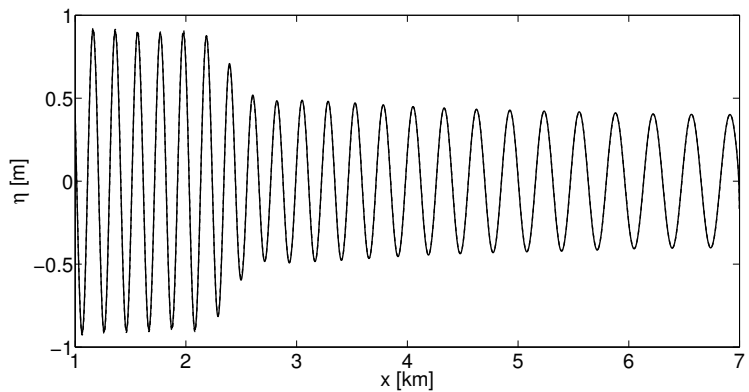

Figure 2.22: Comparison between the simulation in the whole domain (dashed line) and one using the EBC (solid line) for periodic waves with wall reflections. Snapshots at $t=20$ min. The LSWE with WKB2 results are displayed in (a), (c) and LVBM with $D W K B 2$ in (b), (d). 


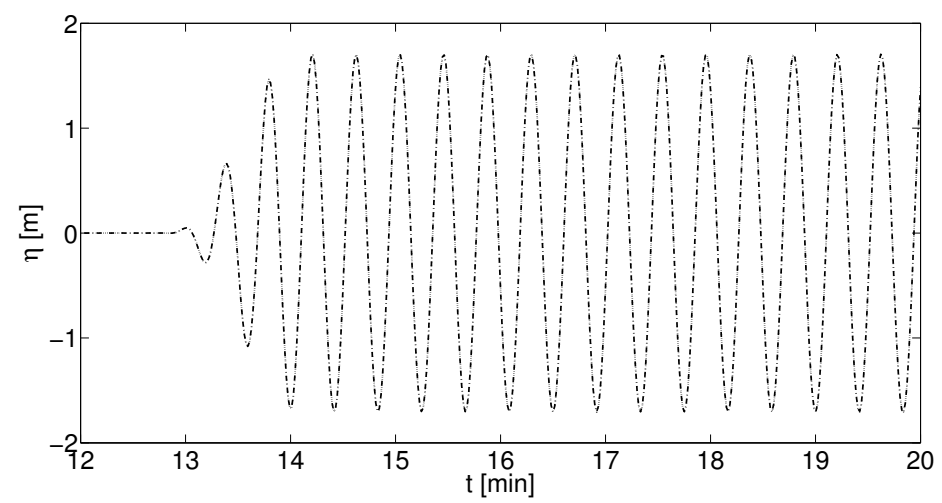

Figure 2.23: Comparison of calculated signals at $x=x_{\mathrm{s}}$ between LSWE-WKB2 (dotteddashed line) and measured signals at $x=x_{\mathrm{s}}$ for a simulation in the full domain with LSWE (dotted line) for periodic waves case.

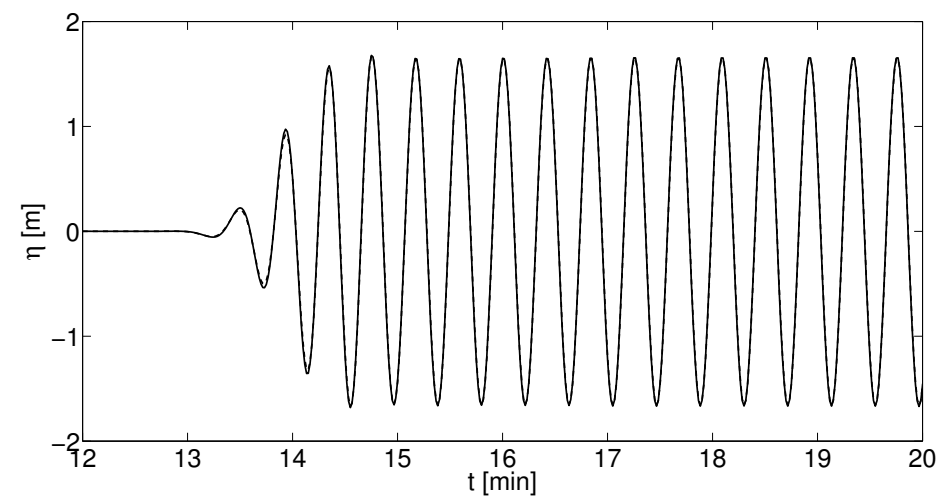

Figure 2.24: Comparison of calculated signals at $x=x_{\mathrm{s}}$ between LVBM-DWKB2 (solid line) and measured signals at $x=x_{\mathrm{s}}$ for a simulation in the full domain with LVBM (dashed line) for periodic waves case. 
zone is sometimes even ignored in numerical models for tsunami prediction and a fully reflective hard-wall boundary condition is used at some shallow depth. In contrast, our EBC is a (partially) reflective, history-dependent boundary condition for an internal boundary. In one dimension normal to the coast line, this internal boundary lies at $x=B$ and the coastline at $x=x_{\mathrm{s}}$. We have defined an observation and influx operators for both models at this internal boundary at $x=B$. The reflection in the model area is derived based on the well-known shallow water theory and the WKB approximation, with extensions to the dispersive variational Boussinesq model. We have integrated this EBC with a finite element treatment in the simulation area and analytical, asymptotic methods in the model area, and have coupled the dynamics using variational principles.

Several test cases have been considered to verify and validate our approach by comparing simulations in the whole domain with ones using the EBC in the smaller simulation domain. These comparisons of LSWE and LVBM show that dispersion effects play an important role and cannot be neglected in simulations of tsunami propagation. As can be seen in Fig. 2.20, for example, the signal measured in the LVBM simulation yields a higher peak wave elevation of about $1 \mathrm{~m}$. In such more dispersive cases, successive wave tails behind the main wave can cause further disaster.

Comparisons of the numerical performances between simulations in the whole domain and simulations using EBC show that our EBC results are competitive in several cases. A comparison of simulation times of all test cases discussed is shown in Table 2.2. The "assembling matrix" times are shorter for all EBC cases, since we reduce the length of our domain in the EBC models. The "ode" times for the EBC models are always shorter than ones in the LSWE simulations, while for LVBM the simulations with EBC are faster except for the flat bottom case. The reason is that in our EBC models, we impose transparent boundary conditions at $x=B$ instead of the hard-wall boundary conditions (natural boundary conditions) for the full simulation. These transparent boundary conditions are essential boundary conditions, adding extra terms in the stiffness matrices. Consequently, the time step used in the ode 45 solver to ensure stability is smaller. It increases the computational time in the first case (where we use hard-wall boundary conditions at the right boundary for the full simulation), while in the second and third cases we have transparent boundary conditions for both cases and therefore the EBC models are faster. In the fourth case, we also have hard-wall boundary conditions at the right boundary for the full simulation, but the increase of computational time due to assembly of the stiffness matrices is compensated by the domain reduction in EBC implementation. In the first case, we only reduce a simple flat bottom in the model area, while in the fourth case we reduce the more complicated nearshore area therefore reducing the simulations time. The 


\begin{tabular}{|c|c|c|c|c|c|c|}
\hline Case & Nx & matrix & ode & trf. & total & ratio \\
\hline Full Flat LSWE & 3000 & 0.21 & 24.38 & - & 24.59 & 0.20 \\
Flat LSWE - WKB0 & 2600 & 0.17 & 21.85 & - & 22.02 & 0.18 \\
Full Flat LVBM & 3000 & 0.63 & 13.95 & - & 14.58 & 0.12 \\
Flat LVBM - DWKB0 & 2600 & 0.50 & 17.93 & - & 18.43 & 0.15 \\
\hline \hline Full Synthetic bath. LSWE & 1387 & 0.02 & 4.76 & - & 4.78 & 0.005 \\
Synth. bath. LSWE - WKB1 & 980 & 0.02 & 3.97 & 0.55 & 4.54 & 0.004 \\
Full Synthetic bath. LVBM & 1387 & 0.16 & 6.29 & - & 6.45 & 0.006 \\
Synth. bath. LVBM - DWKB1 & 980 & 0.10 & 5.08 & 0.95 & 6.13 & 0.005 \\
\hline \hline Full Aceh's bath. LSWE & 4231 & 0.09 & 24.09 & - & 24.18 & 0.003 \\
Aceh's bath. LSWE - WKB1 & 2697 & 0.04 & 18.01 & 0.91 & 18.96 & 0.003 \\
Full Aceh's bath. LVBM & 4231 & 0.68 & 28.89 & - & 29.57 & 0.004 \\
Aceh's bath. LVBM - DWKB1 & 2697 & 0.34 & 18.48 & 2.04 & 20.86 & 0.003 \\
\hline \hline Full Periodic waves LSWE & 2599 & 0.05 & 27.29 & - & 27.34 & 0.02 \\
Periodic waves LSWE - WKB2 & 1999 & 0.03 & 24.03 & 0.08 & 24.14 & 0.02 \\
Full Periodic waves LVBM & 2599 & 0.32 & 21.20 & - & 21.52 & 0.02 \\
Periodic waves LVBM - DWKB2 & 1999 & 0.21 & 19.63 & 0.37 & 20.21 & 0.02 \\
\hline
\end{tabular}

Table 2.2: Table of simulation times for all test cases considered. $N x$ is the number of elements used (equidistant mesh). Column "matrix" shows the time [s] needed for assembling the mass and stiffness matrices. Column "ode" concerns the time spent [s] in the time loop, including computing the observation and influx operator in EBC models. Column "transformation" is the time spent [s] in calculating the coordinate transformation and transfer function (which depends on bathymetric function) in the WKB1, WKB2, DWKB1, and DWKB2 approaches. Column "total" shows the total time [s]. The last column "time ratio" is the fraction of time spent in Matlab's "ode" and the physical time of simulated cases.

"transformation" column shows the time needed for transformation to the new coordinate and calculation of the transfer function in WKB1, WKB2, DWKB1, and DWKB2 approaches, as explained in subsection 2.3.2. The last column "time ratio" is the fraction of time spent in Matlab's "ode" and the physical time of simulated cases. This table shows the strength of our EBC model to reduce the computational time while preserving the accuracy of the results.

The two dimensional extension of the present technique directly follows by numerically integrating a 2D horizontal linear (dispersive) model in the open sea towards the EBC line, after which the analytical 1D theory derived in this article is used for every point on this line. This works provided the waves propagate approximately normal to the EBC (contour) line due to diffraction to the onshore direction via Snell's law. Obviously, the 2D effects in the nearshore area such as 
refraction, focusing, etc. are neglected by applying this approach. Choi et al. 2011] applies this technique and shows that it can be used as a rapid method to predict tsunami run-up heights from 2D numerical simulations. Nevertheless, in Choi et al. (2011), an impermeable boundary condition is used at a $5-10 \mathrm{~m}$ depth at the last sea points instead of a transparent boundary as in the EBC approach. For the future work, we aim to derive the EBC that includes the nonlinear run-up and run-down phenomena on the shore. Note that our variational methodology directly extends to the 2D horizontal and nonlinear case.

\section{Acknowledgements}

We are pleased to acknowledge funding for W.K. from The Netherlands Organization of Scientific Research (NWO), division Earth Sciences. 



\section{Effective Coastal Boundary Conditions for Tsunami Wave Run-Up over Sloping Bathymetry}

\section{Abstract 11:2}

An effective boundary condition (EBC) is introduced as a novel technique for predicting tsunami wave run-up along the coast, and offshore wave reflections. Numerical modeling of tsunami propagation in the coastal zone has been a daunting task, since high accuracy is needed to capture aspects of wave propagation in the shallower areas. For example, there are complicated interactions between incoming and reflected waves due to the bathymetry and intrinsically nonlinear phenomena of wave propagation. If a fixed wall boundary condition is used at a certain shallow depth contour, the reflection properties can be unrealistic. To

\footnotetext{
${ }^{1}$ Previous version of this chapter has been published as:

Kristina, W., Bokhove, O., and van Groesen, E.: Effective coastal boundary conditions for tsunami wave run-up over sloping bathymetry, Nonlin. Processes Geophys. Discuss., 1, 317369, doi:10.5194/npgd-1-317-2014, 2014.

${ }^{2}$ In press:

Kristina, W., Bokhove, O., and van Groesen, E.: Effective coastal boundary conditions for tsunami wave run-up over sloping bathymetry, Nonlin. Processes Geophys., 2014.
} 
alleviate this, we explore a so-called effective boundary condition, developed here in one spatial dimension. From the deep ocean to a seaward boundary, i.e., in the simulation area, we model wave propagation numerically over real bathymetry using either the linear dispersive variational Boussinesq or the shallow water equations. We measure the incoming wave at this seaward boundary, and model the wave dynamics towards the shoreline analytically, based on nonlinear shallow water theory over bathymetry with a constant slope. We calculate the run-up heights at the shore and the reflection caused by the slope. The reflected wave is then influxed back into the simulation area using the EBC. The coupling between the numerical and analytic dynamics in the two areas is handled using variational principles, which leads to (approximate) conservation of the overall energy in both areas. We verify our approach in a series of numerical test cases of increasing complexity, including a case akin to tsunami propagation to the coastline at Aceh, Sumatra, Indonesia.

\subsection{Introduction}

Shallow water equations are widely used in the modeling of tsunamis, since their wavelengths (typically $200 \mathrm{~km}$ ) are far greater than the depth of the ocean (typically 2 to $3 \mathrm{~km}$ ). Tsunamis also tend to have a small amplitude offshore, which is why they generally are less noticeable at sea. Linear shallow water equations (LSWE) therefore often suffice as a simple model of tsunami propagation Choi et al., 2011, Liu et al., 2009]. On the contrary, it turns out that the lack of dispersion is a shortcoming of shallow water modeling when the tsunami reaches the shallower coastal waters on the continental shelf, and thus dispersive models are often required [Madsen et al., 1991, Horrillo et al., 2006]. Numerical simulations based on these linear models are desirable because they involve a small amount of computation. However, as the tsunami approaches the shore, shoaling effects cause a decrease in the wavelength and an increase in the amplitude. Here, the nonlinearity starts to play a more important role, and thus the nonlinear terms must be included in the model. To capture these shoaling effects in more detail, a smaller grid size will be needed. Consequently, longer computational times are required.

Some numerical models of tsunamis use nested methods with different mesh resolutions to preserve the accuracy of the solution near the coastal area Titov et al., 2011, Wei et al., 2008, Kânoğlu and Synolakis, 1998, while other models employ an impenetrable vertical wall at a certain depth contour as the boundary condition. Obviously, the reflection properties of such a boundary condition can be unrealistic. We therefore wish to alleviate this shortcoming by an investigation of a so-called effective boundary condition (EBC) [Kristina et al., 2012], 


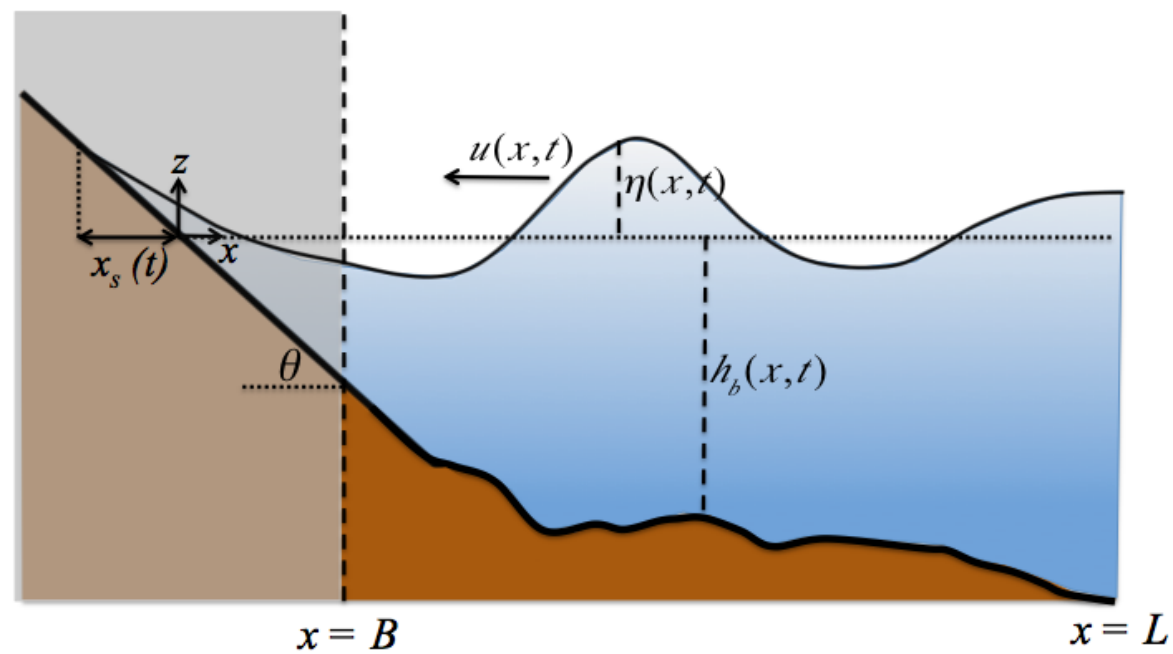

Figure 3.1: At the seaward boundary $x=B$, we assign $(\eta, u)$-data, and we want to find a solution of the NSWE on the sloping region near the shoreline.

including run-up. In one horizontal spatial dimension, an outline of the desired mathematical modeling is sketched in Fig. 3.1. In the deep ocean for $x \in[B, L]$ with horizontal coordinate $x$ and seaward boundary point $x=B$, denoted as the simulation area, we model the wave propagation numerically using a linear model. In the coastal zone for $x \in\left[x_{\mathrm{s}}(t), B\right]$ with shoreline position $x_{\mathrm{s}}(t)<B$, denoted as the model area, we model the wave propagation analytically using a nonlinear model by approximating the bathymetry as a planar beach. We calculate the run-up heights at the shore and the reflection caused by the slope. The reflected wave is then influxed back into the simulation area using the EBC. The coupling between the numerical and analytic dynamics in the two areas is handled using variational principles, which leads to (approximate) conservation of the overall energy in both areas. Following Kristina et al. [2012], an observation and influx operator are defined at $x=B$ to measure the incoming wave signal and to influx the reflected wave, respectively.

The shoreline position and wave reflection in the model area (sloping region) are determined using an analytical solution of the nonlinear shallow water equations (NSWE) following the approach of Antuono and Brocchini [2010] for unbroken waves. The decomposition of the incoming wave signal and the reflected one is also described in Antuono and Brocchini (2007, 2010) for the calculation of the shoreline and the wave reflection. Nevertheless, the method in their paper is applied by determining the incoming wave signal with the solution of the Korteweg-de Vries (KdV) equation. The novelty of our approach is the utilization 
of an observation operator at the boundary $x=B$ to calculate the incoming wave elevation towards the shore from the numerical solution of the LSWE in the simulation area. For any given wave profile and bathymetry in the simulation area, the numerical solution can be calculated, and the signal arriving at $x=B$ can be observed. Afterwards, the data are used to calculate the analytical solution of the NSWE in the onshore region and the reflected waves.

A rapid method for estimating tsunami run-up heights is also suggested by Choi et al. 2011, 2012 by imposing a hard-wall boundary condition at $x=B$. Giving the water wave oscillations at this hard wall at $x=B$, the maximum runup height of tsunami waves at the coast is subsequently calculated separately by employing a linear approach. It is claimed that the linear and nonlinear theories predict the same maximal values for the run-up height if the incident wave is determined far from the shore [Synolakis, 1987]. In contrast, Li and Raichlen 2001] show that there is a difference in the maximum run-up prediction between linear and nonlinear theory. In addition to calculating only the maximum run-up height as in Choi's method, our EBC also includes the calculation of reflected waves. The point-wise wave height in the whole domain (offshore and onshore area) is thus predicted accurately. For the inundation prediction, we have verified that the method introduced by Choi et al. (2011, 2012) performs as well as our EBC method, while reflection wave comparisons show larger discrepancies due to the usage of a hard-wall boundary condition. The interaction between incoming and reflected waves needs to be predicted accurately, since subsequent waves may cause danger at later times. Stefanakis et al. [2011] investigate the fact that resonant phenomena between the incident wavelength and the beach slope are found to occur. The resonance happens due to incoming and reflected wave interactions, and the actual amplification ratio depends on the beach slope. It explains why in some cases it is not the first wave that results in the highest run-up.

Determination of the location of the seaward boundary point $x=B$ is another issue that must be addressed. Choi et al. 2011] put the impermeable boundary conditions at a 5-10 m depth contour. In comparison, Didenkulova and Pelinovsky [2008] show that their run-up formula for symmetric waves gives optimal results when the incoming wave signal is measured at a depth that is two-thirds of the maximum wave height. We determine the location of this seaward boundary as the point before the nonlinearity effect arises, and examine the dispersion effect at that point as well. Considering the simple KdV equation [Mei, 1989], the measures of nonlinearity and dispersion are given by the ratios $\epsilon=A / h$ and $\mu^{2}=(k h)^{2}$ for the wave amplitude $A$, water depth $h$, and wavenumber $k$. Provided with the information of the initial wave profile, we can calculate the amplification of the amplitude and the decrease in the wavelength in a linear 
approach, and thereafter estimate the location of the EBC point.

The EBC in this article will be derived in one spatial dimension for reasons of simplicity and clarity of exposure. The numerical solution in the simulation area is based on a variational finite element method (FEM). In order to verify the EBC implementation that employs this asymptotic closed-form solution, we also numerically simulate the NSWE in the model area using a finite volume method (FVM). Both cases are coupled to the simulation area to compare the results. We also validate our approach against the laboratory experiment of Synolakis [1987]. In Sect. 3.2, we introduce the linear variational Boussinesq model (LVBM) and shallow water equations (SWE), both linear and nonlinear, from their variational principles. The coupling conditions required at the seaward boundary point are also derived here. The solution of the NSWE using a method of characteristics is shown in Sect. 3.3, which includes the solution of the shoreline position. In Sect. 3.4, the effective boundary condition is derived. It pinpoints the newly derived coupling conditions between the finite element simulation area and the model area. Numerical validation and verification are shown in Sect. 3.5, and we conclude in Sect. 3.6 .

\subsection{Water wave models}

Our primary goal is to model the water wave motion to the shore analytically, instead of resolving the motion in these shallow regions numerically. We therefore introduce an artificial open boundary at some depth, and wish to determine an effective boundary condition at this internal boundary. Consider motion in a vertical plane normal to the shore, with an offshore coordinate $x$. The artificial boundary is then placed at $x=B$, while the real (time-dependent) boundary lies at $x=x_{\mathrm{s}}(t)$ with $x_{\mathrm{s}}(t)<B$. For example, at rest, land starts at $x=0$, where the total water depth $h(0, t)$ is zero. This water line is time dependent, as the wave can move up and down the beach.

We will restrict our attention to the dynamics in a vertical plane with horizontal and vertical coordinates $x$ and $z$, respectively. Nonlinear potential flow water waves are succinctly described by the variational principles as follows Luke, 1967, Zakharov, 1968, Miles, 1977]:

$$
\begin{aligned}
0 & =\delta \int_{0}^{T} \mathcal{L}\left[\phi, \Phi, \eta, x_{\mathrm{s}}\right] \mathrm{d} t \\
& =\delta \int_{0}^{T} \int_{x_{\mathrm{s}}}^{L}\left(\phi \partial_{t} \eta-\frac{1}{2} g\left((h+b)^{2}-b^{2}\right)-\int_{-h_{\mathrm{b}}}^{\eta} \frac{1}{2}|\nabla \Phi|^{2} \mathrm{~d} z\right) \mathrm{d} x \mathrm{~d} t
\end{aligned}
$$

with velocity potential $\Phi=\Phi(x, z, t)$ and surface potential $\phi(x, t)=\Phi(x, z=$ $\eta, t)$, where $\eta=h-h_{\mathrm{b}}$ is the wave elevation and $h=h(x, t)$ the total water 
depth above the bathymetry $b=-h_{\mathrm{b}}(x)$, with $h_{\mathrm{b}}(x)$ the rest depth. Time runs from $t \in[0, T]$; partial derivatives are denoted by $\partial_{t}$, et cetera, the gradient in the vertical plane by $\nabla=\left(\partial_{x}, \partial_{z}\right)^{T}$, and the acceleration of gravity by $g$.

The approximation for the velocity potential $\Phi$ in Eq. (3.1) can be of various kinds, but all are based on the idea of restricting the class of wave motions to a class that contains the wave motions one is interested in van Groesen, 2006, Cotter and Bokhove, 2010, Gagarina et al., 2013. Following Klopman et al. [2010], we approximate the velocity potential as follows

$$
\Phi(x, z, t)=\phi(x, t)+F(z) \psi(x, t)
$$

for a function $F=F(z)$. Its suitability is determined by insisting that $F(\eta)=$ 0 , such that $\phi$ is the potential at the location $z=\eta$ of the free surface and satisfies the slip flow condition at the bottom boundary $z+h_{\mathrm{b}}(x)=0$. The latter kinematic condition yields $\partial_{z} \Phi+\partial_{x} \Phi \partial_{x} h_{\mathrm{b}}=0$ at $z=-h_{\mathrm{b}}(x)$. For a slowly varying bottom topography, this condition is approximated by

$$
\left(\partial_{z} \Phi\right)_{z=-h_{\mathrm{b}}(x)}=F^{\prime}\left(-h_{\mathrm{b}}\right) \psi=0 .
$$

Substitution of Eq. (3.2) into Eq. (3.1) yields the variational principle for Boussinesq equations, as follows Klopman et al. 2010.

$$
\begin{aligned}
0=\delta \int_{0}^{T} \mathcal{L}\left[\phi, \psi, \eta, x_{\mathrm{s}}\right] \mathrm{d} t \\
=\delta \int_{0}^{T} \int_{x_{\mathrm{s}}}^{L}\left(\phi \partial_{t} \eta-\frac{1}{2} g\left((h+b)^{2}-b^{2}\right)-\frac{1}{2}\left(\eta+h_{\mathrm{b}}\right)\left|\partial_{x} \phi\right|^{2}\right. \\
\left.\quad-\breve{\beta} \partial_{x} \psi \partial_{x} \phi-\frac{1}{2} \breve{\alpha}\left|\partial_{x} \psi\right|^{2}-\frac{1}{2} \breve{\gamma} \psi^{2}\right) \mathrm{d} x \mathrm{~d} t,
\end{aligned}
$$

where functions $\breve{\beta}(x), \breve{\alpha}(x)$, and $\breve{\gamma}(x)$ are given by

$$
\breve{\beta}(x)=\int_{-h_{\mathrm{b}}}^{\eta} F \mathrm{~d} z, \quad \breve{\alpha}(x)=\int_{-h_{\mathrm{b}}}^{\eta} F^{2} \mathrm{~d} z, \quad \breve{\gamma}(x)=\int_{-h_{\mathrm{b}}}^{\eta}\left(F^{\prime}\right)^{2} \mathrm{~d} z .
$$

The shallow water equations (SWE) are derived with the assumption that the wavelengths of the waves are much larger than the depth of the fluid layer, so that the vertical variations are small and will be ignored. In this case, there is no dispersive effect. The velocity potential is approximated over depth by its value at the surface, such that $F(z)=0$. Hence, when $\breve{\beta}=\breve{\alpha}=\breve{\gamma}=0$ in Eq. 3.4, the nonlinear shallow water equations are obtained as a limiting system.

We a priori divide the domain into two intervals: $x \in[B, L]$, where we model the wave propagation linearly, and $x \in\left[x_{\mathrm{s}}(t), B\right]$, where we keep the nonlinearity. 
To be precise, in the simulation area $x \in[B, L]$, we linearize the equations, and thus the wave propagation in this domain is modeled by the linear shallow water shallow water equations or the linear yet dispersive Boussinesq model. In the model area $x \in\left[x_{s}(t), B\right]$, we only consider depth-averaged shallow water flow. The non-dispersive and nonlinear shallow water equations are thus used to model the wave propagation in this region. Hereafter, we write $\breve{\phi}$ and $\breve{\eta}$ for the linear variables and also the definitions of $\breve{\beta}, \breve{\alpha}$ and $\breve{\gamma}$ simplify accordingly. Consequently, by applying the corresponding approximations to variational principle (3.4), the (approximated) variational principle becomes

$$
\begin{aligned}
0=\delta \int_{0}^{T} \mathcal{L}\left[\breve{\phi}, \breve{\psi}, \breve{\eta}, \phi, \eta, x_{\mathrm{s}}\right] \mathrm{d} t \\
=\delta \int_{0}^{T}\left[\int_{B}^{L}\left(\breve{\phi} \partial_{t} \breve{\eta}-\frac{1}{2} g \breve{\eta}^{2}-\frac{1}{2} h_{\mathrm{b}}\left|\partial_{x} \breve{\phi}\right|^{2}-\breve{\beta} \partial_{x} \breve{\psi} \partial_{x} \breve{\phi}-\frac{1}{2} \breve{\alpha}\left|\partial_{x} \breve{\psi}\right|^{2}-\frac{1}{2} \breve{\gamma} \breve{\psi} \breve{\psi}^{2}\right) \mathrm{d} x\right. \\
\left.\quad+\int_{x_{\mathrm{s}}}^{B}\left(\phi \partial_{t} \eta-\frac{1}{2} g\left((h+b)^{2}-b^{2}\right)-\frac{1}{2}\left(\eta+h_{\mathrm{b}}\right)\left|\partial_{x} \phi\right|^{2}\right) \mathrm{d} x\right] \mathrm{d} t .
\end{aligned}
$$

We choose a parabolic profile function $F\left(z ; h_{\mathrm{b}}\right)=2 z / h_{\mathrm{b}}+z^{2} / h_{\mathrm{b}}^{2}$, in which the $x$ dependence is considered to be parametric when total water depth $h$ is sufficiently slowly varying. The coefficients in (3.5) simplify to their linearized counterparts in the simulation area where the linear Boussinesq equations hold (while these coefficients disappear in the model area where the nonlinear depthaveraged shallow water equations hold)

$$
\begin{aligned}
& \breve{\alpha}=\breve{\alpha}(x)=\int_{-h_{\mathrm{b}}}^{0} F^{2} \mathrm{~d} z=\frac{8}{15} h_{\mathrm{b}}, \\
& \breve{\beta}=\breve{\beta}(x)=\int_{-h_{\mathrm{b}}}^{0} F \mathrm{~d} z=-\frac{2}{3} h_{\mathrm{b}}, \\
& \breve{\gamma}=\breve{\gamma}(x)=\int_{-h_{\mathrm{b}}}^{0}\left(F^{\prime}\right)^{2} \mathrm{~d} z=\frac{4}{3 h_{\mathrm{b}}} .
\end{aligned}
$$

The variations in Eqs. (3.6) yield

$$
\begin{array}{rl}
0=\lim _{\epsilon \rightarrow 0} \frac{1}{\epsilon} \int_{0}^{T} & \mathcal{L}\left[\breve{\phi}+\epsilon \delta \breve{\phi}, \breve{\psi}+\epsilon \delta \breve{\psi}, \breve{\eta}+\epsilon \delta \breve{\eta}, \phi+\epsilon \delta \phi, \eta+\epsilon \delta \eta, x_{\mathrm{s}}+\epsilon \delta x_{\mathrm{s}}\right] \\
& -\mathcal{L}\left[\breve{\phi}, \breve{\psi}, \breve{\eta}, \phi, \eta, x_{\mathrm{s}}\right] \mathrm{d} t
\end{array}
$$




$$
\begin{aligned}
& =\int_{0}^{T}\left[\int _ { B } ^ { L } \left(\left(\partial_{t} \breve{\eta}+\partial_{x}\left(h_{\mathrm{b}} \partial_{x} \breve{\phi}\right)+\partial_{x}\left(\breve{\beta} \partial_{x} \breve{\psi}\right)\right) \delta \breve{\phi}-\left(\partial_{t} \breve{\phi}+g \breve{\eta}\right) \delta \breve{\eta}\right.\right. \\
& \left.+\left(\partial_{x}\left(\breve{\alpha} \partial_{x} \breve{\psi}\right)+\partial_{x}\left(\breve{\beta} \partial_{x} \breve{\phi}\right)-\breve{\gamma} \breve{\psi}\right) \delta \breve{\psi}\right) \mathrm{d} x \\
& +\underline{\left.\left(h_{\mathrm{b}} \partial_{x} \breve{\phi}+\breve{\beta} \partial_{x} \breve{\psi}\right) \delta \breve{\phi}\right|_{x=B}+\left.\left(\breve{\alpha} \partial_{x} \breve{\psi}+\breve{\beta} \partial_{x} \breve{\phi}\right) \delta \breve{\psi}\right|_{x=B}} \\
& +\int_{x_{\mathrm{s}}}^{B}\left(\left(\partial_{t} \eta+\partial_{x}\left(\left(\eta+h_{\mathrm{b}}\right) \partial_{x} \phi\right)\right) \delta \phi-\left(\partial_{t} \phi+g \eta+\frac{1}{2} \partial_{x}^{2} \phi\right) \delta \eta\right) \mathrm{d} x \\
& \left.-\underline{\left.\left(\eta+h_{\mathrm{b}}\right) \partial_{x} \phi \delta \phi\right|_{x=B}}+\left.(\phi \delta \eta)\right|_{x=x_{\mathrm{s}}} \frac{\mathrm{d} x_{\mathrm{s}}}{\mathrm{d} t}-\left.\left(\phi \partial_{t} \eta\right)\right|_{x=x_{\mathrm{s}}} \delta x_{\mathrm{s}}\right] \mathrm{d} t,
\end{aligned}
$$

where we used the endpoint conditions $\delta \eta(0)=\delta \eta(T)=0$, and no-normal through-flow conditions at $x=L$ and $h\left(x_{\mathrm{s}}(t), t\right)=0$. Since the variations are arbitrary, the linear equations emerging from Eq. $(3.8 \mathrm{~b})$ for $x \in[B, L]$ are as follows:

$$
\begin{aligned}
& \partial_{t} \breve{\phi}+g \breve{\eta}=0, \\
& \partial_{t} \breve{\eta}+\partial_{x}\left(h_{\mathrm{b}} \partial_{x} \breve{\phi}\right)+\partial_{x}\left(\breve{\beta} \partial_{x} \breve{\psi}\right)=0 \\
& \partial_{x}\left(\breve{\alpha} \partial_{x} \breve{\psi}\right)+\partial_{x}\left(\breve{\beta} \partial_{x} \breve{\phi}\right)-\breve{\gamma} \breve{\psi}=0
\end{aligned}
$$

and for $x \in\left[x_{\mathrm{s}}(t), B\right]$, we get the nonlinear equations of motion

$$
\begin{aligned}
& \partial_{t} \phi+g \eta+\frac{1}{2} \partial_{x}^{2} \phi=0, \\
& \partial_{t} \eta+\partial_{x}\left(\left(\eta+h_{\mathrm{b}}\right) \partial_{x} \phi\right)=0 .
\end{aligned}
$$

The last two terms in Eq. (3.8b are the boundary terms at $x=x_{\mathrm{s}}$. They can be rewritten as follows:

$$
\int_{0}^{T}\left[\left.(\phi \delta \eta)\right|_{x=x_{\mathrm{s}}} \frac{\mathrm{d} x_{\mathrm{s}}}{\mathrm{d} t}-\left.\left(\phi \partial_{t} \eta\right)\right|_{x=x_{\mathrm{s}}} \delta x_{\mathrm{s}}\right] \mathrm{d} t=\int_{0}^{T}\left[\left(-\phi \partial_{x}\left(\eta+h_{\mathrm{b}}\right) \frac{\mathrm{d} x_{\mathrm{s}}}{\mathrm{d} t}-\phi \partial_{t} \eta\right) \delta x_{\mathrm{s}}\right]_{x=x_{\mathrm{s}}} \mathrm{d} t,
$$

since the total depth is $h\left(x_{\mathrm{s}}, t\right)=\eta\left(x_{\mathrm{s}}, t\right)+h_{\mathrm{b}}\left(x_{\mathrm{s}}\right)=0$ at the shoreline boundary. We therefore have the relation $0=\delta h\left(x_{\mathrm{s}}, t\right)=\delta h+\partial_{x} h \delta x_{\mathrm{s}}=\delta \eta+\partial_{x}\left(\eta+h_{\mathrm{b}}\right) \delta x_{\mathrm{s}}$. Substituting Eq. (3.10b) into (3.11), the boundary condition at the shoreline is

$$
\frac{\mathrm{d} x_{\mathrm{s}}}{\mathrm{d} t}=\partial_{x} \phi \text { at } x=x_{\mathrm{s}}(t)
$$

i.e., the velocity of the shoreline equals the horizontal velocity of the fluid particle. The underlined terms in Eq. $3.8 \mathrm{~b}$ apply at the seaward point, where we want to derive the coupling of effective boundary conditions. To derive the condition 
for the linear model, the goal is to write these terms using the variations $\delta \breve{\phi}$ and $\delta \breve{\psi}$. Because the depth-averaged shallow water equations are considered, we have

$$
\phi(x, t)=\bar{\Phi}(x, t)=\frac{1}{h_{\mathrm{b}}} \int_{-h_{\mathrm{b}}}^{0} \Phi(x, z, t) \mathrm{d} z=\breve{\phi}+\frac{\breve{\beta}}{h_{\mathrm{b}}} \breve{\psi},
$$

where the last equality arises from approximation 3.2 for the velocity potential. The variation of $\delta \phi$ thus becomes

$$
\delta \phi=\delta \breve{\phi}+\frac{\breve{\beta}}{h_{\mathrm{b}}} \delta \breve{\psi} .
$$

Substituting this into Eq. 3.8b), we get the coupling condition at $x=B$ for the linear model as follows:

$$
\begin{aligned}
h_{\mathrm{b}} \partial_{x} \breve{\phi}+\breve{\beta} \partial_{x} \breve{\psi} & =h \partial_{x} \phi \\
\breve{\alpha} \partial_{x} \breve{\psi}+\breve{\beta} \partial_{x} \breve{\phi} & =\frac{\breve{\beta}}{h_{\mathrm{b}}} h \partial_{x} \phi
\end{aligned}
$$

To derive the condition for the nonlinear shallow water model, we use the approximation for the velocity potential (3.2) again. Since $F(z=\eta)=0$ at the surface, we have $\phi=\breve{\phi}$ and thus $\delta \phi=\delta \breve{\phi}$. From Eq. (3.8b), the coupling condition for a nonlinear model is given by

$$
h \partial_{x} \phi=h_{\mathrm{b}} \partial_{x} \breve{\phi}+\breve{\beta} \partial_{x} \breve{\psi} .
$$

Note that the coupling conditions $3.15-(3.16)$ are used to transfer the information between the two domains. Coupling condition 3.15 gives the information of $\breve{\phi}$ and $\breve{\psi}$ in the simulation area, provided the information of $\phi$ from the model area is given. Meanwhile, coupling condition $\sqrt{3.16}$ gives the information of $\phi$ in the model area, provided the information of $\breve{\phi}$ and $\breve{\psi}$ from the simulation area is given.

\subsection{Nonlinear Shallow Water Equations}

\subsubsection{Characteristic form}

We will start with the NSWE in the shore region. Using $\eta=-h_{\mathrm{b}}+h$ and velocity $u=\partial_{x} \phi$, we may rewrite Eq. (3.10) as follows (starred variables are used here for later convenience):

$$
\begin{aligned}
\partial_{t^{\star}} h^{\star}+\partial_{x^{\star}}\left(h^{\star} u^{\star}\right) & =0 \\
\partial_{t^{\star}} u^{\star}+u^{\star} \partial_{x^{\star}} u^{\star} & =-g^{\star} \partial_{x^{\star}}\left(-h_{\mathrm{b}}^{\star}+h^{\star}\right) .
\end{aligned}
$$


The dimensionless form of Eq. (3.17) for a still water depth $h_{\mathrm{b}}^{\star}=\gamma^{\star} x^{\star}$ (where $\gamma^{\star}=\tan \theta$ is the beach slope) is obtained by using the scaling factors Brocchini and Peregrine, 1996

$$
h=\frac{h^{\star}}{h_{0}}, u=\frac{u^{\star}}{u_{0}}, x=\frac{x^{\star}}{l_{0}}, t=\frac{t^{\star}}{t_{0}},
$$

in which $h_{0}$ is the still water depth at the seaward boundary, and $u_{0}, l_{0}$, and $t_{0}$ are defined below as

$$
u_{0}=\sqrt{\frac{g^{\star} h_{0}}{g}}, l_{0}=\frac{h_{0} \gamma}{\gamma^{\star}}, t_{0}=\frac{\gamma}{\gamma^{\star}} \sqrt{\frac{g h_{0}}{g^{\star}}},
$$

where $g=1$ and $\gamma=1$ are dimensionless gravity acceleration and beach slope, respectively. The NSWE in dimensionless form are then given by

$$
\begin{aligned}
\partial_{t} h+\partial_{x}(h u) & =0 \\
\partial_{t} u+u \partial_{x} u & =g \gamma-g \partial_{x} h .
\end{aligned}
$$

The asymptotic solution of this system of equations for wave propagation over sloping bathymetry has been given for several initial-value problems using a hodograph transformation [Carrier and Greenspan, 1958, Synolakis, 1987, Pelinovsky and Mazova, 1992, Carrier et al., 2003, Kânoğlu, 2004, and also for the boundary-value problem Antuono and Brocchini, 2007, Li and Raichlen, 2001, Madsen and Schäffer, 2010 that will used in this article. Since the system is hyperbolic, it has the following characteristic forms:

$$
\begin{gathered}
\frac{\mathrm{d} \alpha}{\mathrm{d} t}=0 \text { on } \frac{\mathrm{d} x}{\mathrm{~d} t}=u-c \\
\frac{\mathrm{d} \beta}{\mathrm{d} t}=0 \text { on } \frac{\mathrm{d} x}{\mathrm{~d} t}=u+c
\end{gathered}
$$

in which $c=\sqrt{g h}$,

$$
\alpha=2 c-u+g \gamma t, \quad \text { and } \quad \beta=2 c+u-g \gamma t .
$$

Variables $\alpha$ and $\beta$ are the so-called Riemann invariants, since they do not change their value along the characteristic curves in Eq. (3.21). Assuming the flow to be subcritical (that is, $|u|<c$ ), the first characteristic curves with $u-c<0$ are called "incoming", since they propagate signals towards the shore. The second ones with $u+c>0$ are called "outgoing", since they move towards the deeper waters (carrying information on the wave reflection over the sloping region). 


\subsubsection{A trivial solution of the characteristic curve}

In the trivial case of no motion $(u=\eta \equiv 0)$, as well as in the dynamic case presented later, we focus on the incoming characteristic curve. In the rest case, it is given by

$$
\frac{\mathrm{d} x}{\mathrm{~d} t}=-\sqrt{g \gamma x} .
$$

For $x \neq 0$, substituting $y=\sqrt{g \gamma x}$ results in the general solution for variable $y$ as follows:

$$
y=-\frac{1}{2} g \gamma t+C_{2},
$$

with a constant $C_{2}$. When the curve intersects $x=B$ at time $\tau$, with $h_{0}$ the depth at $x=B$, such that $h_{0}=\gamma B$ and $y(B)=\sqrt{g \gamma B}=c_{0}$, the particular solution is given by

$$
y=\frac{2 c_{0}-g \gamma(t-\tau)}{2} .
$$

In the case of no motion, the boundary data $\alpha=\alpha_{0}(\tau)$ and $\beta=\beta_{0}(\tau)$ are as follows:

$$
\alpha_{0}=2 c_{0}+g \gamma \tau, \quad \beta_{0}=2 c_{0}-g \gamma \tau .
$$

Transforming back to the $x$ variable, while using these expressions, we get the incoming characteristic curve

$$
x=\frac{1}{4 g \gamma}\left(g \gamma t-\alpha_{0}\right)^{2}=\frac{g \gamma(2 \omega-(t-\tau))^{2}}{4}
$$

with $\omega=c_{0} /(g \gamma)$. Along this characteristic curve, the Riemann invariant is constant.

Figure 3.2 shows the characteristic curves of the dimensionless NSWE over sloping bathymetry $b(x)=-x$ for $x \in[0,1]$, and LSWE over flat bathymetry $h_{0}=1, B=1$ for $x \in[1,2]$. As in our previous paper [Kristina et al., 2012], the characteristic curves of the LSWE are given by $\mathrm{d} x / \mathrm{d} t= \pm c_{0}$. The "incoming" and "outgoing" characteristic curves are shown by the solid and dashed lines, respectively.

For each characteristic curve (3.27), the location of the shoreline can be determined by looking for the $\tau=\tau_{\mathrm{s}}$ for which the characteristic reaches the shoreline position, here $x=0$, at time $t$. It is given by the condition

$$
\frac{\partial x}{\partial \tau}=0 \text { so that } \tau_{\mathrm{s}}=t-2 \omega .
$$




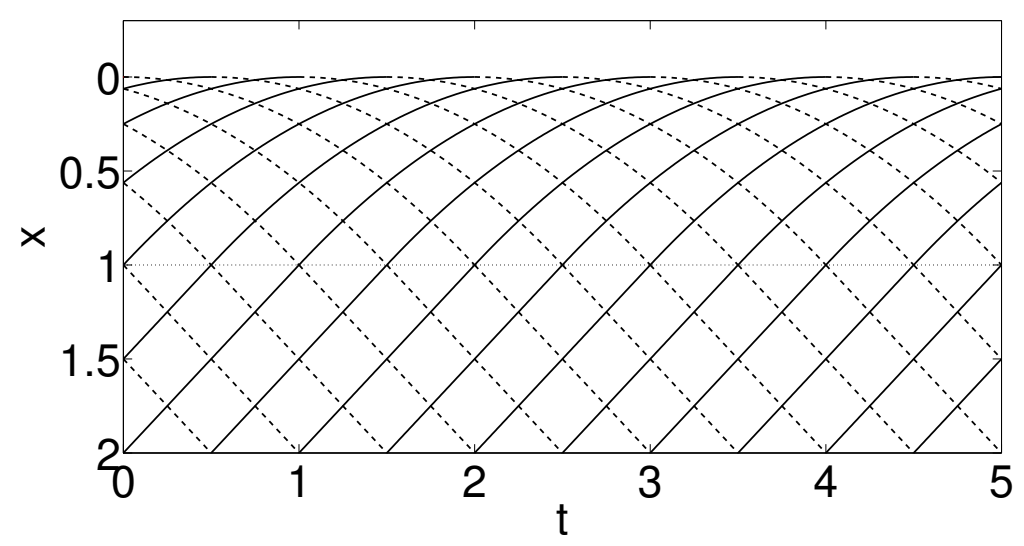

Figure 3.2: Plot of the characteristic curves in the case of no motion ( $\eta=u=0$ ) for the dimensionless NSWE over sloping bathymetry $b(x)=-x$ for $x \in[0,1]$ and LSWE over flat bathymetry $h_{0}=1, B=1$ for $x \in[1,2]$. The "incoming" and "outgoing" characteristic curves are shown by solid and dashed lines, respectively. The shoreline $x=0$ can be seen as the envelopes of the characteristic curves themselves.

As displayed in Fig. 3.2, the incoming characteristic curves that reach the shoreline at time $t$ intersect $x=B=1$ at time $\tau=t-2(\omega=1$ in this case). Since $u$ equals zero in the rest case, the boundary condition $(3.12)$ is of course satisfied.

\subsubsection{Boundary Value Problem (BVP)}

Li and Raichlen 2001 and Synolakis 1987 combine linear and nonlinear theory to reduce the difficulties in the assignment of the boundary data for solving the BVP problem in the NSWE. Later, it is shown that the proper way to solve the assignment problem without using linear theory at all is not given in terms of $\eta$ or $u$ (Antuono and Brocchini, 2007), but in terms of the incoming Riemann variable $\alpha$. This article follows the approach of Antuono and Brocchini [2010], who use this incoming Riemann variable as boundary data and solve the dimensionless NSWE by direct use of physical variables instead of using the hodograph transformation introduced by Carrier and Greenspan [1958. We do, however, clarify the mathematics of the boundary condition at the shoreline.

Given the data of $\eta$ and $u$ at the seaward boundary $x=B$, for all time $t$, we want to find a solution of the NSWE in the sloping region to the shoreline, including the reflected waves traveling back into the deeper waters. If the sea is assumed in the rest state during the initial condition, the data are $\eta(B, t)=u(B, t)=0$ for $t<0$. In accordance to the previous trivial case, the initial time where a characteristic meets $x=B$ is labeled as $\tau$, and we write $x=\chi(t, \tau)$, so we have the 
data $\alpha=\alpha_{0} \equiv 2 c(B, \tau)-u(B, \tau)+g \gamma \tau$ along the incoming characteristic curves and $\beta=\beta_{0} \equiv 2 c(B, \tau)+u(B, \tau)-g \gamma \tau$ along the outgoing characteristic curves. We can then rewrite Eq. (3.21) as

$$
\begin{aligned}
& \alpha=\alpha_{0} \text { on curves such that } \chi_{t}=u-c=\frac{\beta-3 \alpha_{0}}{4}+g \gamma t \\
& \beta=\beta_{0} \text { on curves such that } \chi_{t}=u+c=\frac{3 \beta_{0}-\alpha}{4}+g \gamma t,
\end{aligned}
$$

which means that the boundary values are carried by the incoming and outgoing characteristic curves. To be concise, we write $\chi_{t}=\partial_{t} \chi$ and $\chi_{\tau}=\partial_{\tau} \chi$. Our aim is to obtain a closed equation for the dynamics, and we focus on the incoming characteristic by fixing $\alpha=\alpha_{0}$. We can rewrite Eq. 3.29a as follows:

$$
\beta=3 \alpha_{0}+4\left(\chi_{t}-g \gamma t\right) .
$$

Here, $\beta=\beta(\chi, t)$, since we are moving along an incoming characteristic curve. By taking the total $t$ derivative of $\beta$, we obtain

$$
\frac{\mathrm{d} \beta}{\mathrm{d} t}=\beta_{t}+\beta_{x} \chi_{t}=\beta_{t}+\left(\frac{\beta-3 \alpha_{0}}{4}+g \gamma t\right) \beta_{x}=4\left(\chi_{t t}-g \gamma\right),
$$

in which the last equality comes from Eq. 3.30). In addition, the $\tau$-derivative of Eq. 3.30 gives

$$
\frac{\partial \beta}{\partial \tau}=\beta_{x} \chi_{\tau}=3 \dot{\alpha}_{0}+4 \chi_{t \tau} \Rightarrow \beta_{x}=\frac{3 \dot{\alpha}_{0}+4 \chi_{t \tau}}{\chi_{\tau}},
$$

in which $\dot{\alpha}_{0}=\partial_{\tau} \alpha_{0}$. We still need an explicit expression for $\beta_{t}$, which can be obtained by rewriting Eq. $3.21 \mathrm{~b}$ in the following way:

$$
\beta_{t}+\left(\frac{3 \beta-\alpha_{0}}{4}+g \gamma t\right) \beta_{x}=0 .
$$

Combining Eqs. 3.31)-3.33), we get the following differential equation for the incoming characteristic curves:

$$
2 \chi_{\tau}\left(\chi_{t t}-g \gamma\right)=\left(4 \chi_{t \tau}+3 \dot{\alpha}_{0}\right)\left(g \gamma t-\alpha_{0}-\chi_{t}\right) \text { for } t>\tau
$$

with boundary conditions

$$
\begin{aligned}
\left.\chi\right|_{t=\tau} & =B \\
\left.\chi_{\tau}\right|_{\tau=\tau_{\mathrm{s}}} & =0 .
\end{aligned}
$$

The second boundary condition is the shoreline boundary condition. We have $4 c=\alpha+\beta$ from Eq. (3.22), which implies $\beta=-\alpha$ at the shoreline $c=0$. Using Eq. (3.30), we note that $4 c=\alpha_{0}+\beta=4\left(\alpha_{0}+\chi_{t}-g \gamma t\right)=0$ at the shoreline. Hence, the right-hand side of Eq. 3.34a is zero, such that for consistency $\chi_{\tau}$ must be zero at the shoreline, since generally, $\chi_{t t} \neq g \gamma$. 


\section{Perturbation expansion}

Due to the nonlinearity in $\chi$, we use a perturbation method to solve Eq. (3.34). We expand it in a perturbation series around the rest solution (3.27), with the assumption of small data at $x=B$. Using the linearity ratio $\epsilon=A / h_{0}(A$ is the wave amplitude), we say a wave is small if $\epsilon \ll 1$, and expand as follows:

$$
\begin{aligned}
\alpha_{0} & =\alpha_{0,0}+\epsilon \alpha_{0,1}+\mathcal{O}\left(\epsilon^{2}\right), \\
\chi & =\chi^{(0)}+\epsilon \chi^{(1)}+\mathcal{O}\left(\epsilon^{2}\right), \\
\tau_{\mathrm{s}} & =\tau_{0}(t)+\epsilon \tau_{1}(t)+\mathcal{O}\left(\epsilon^{2}\right),
\end{aligned}
$$

in which $\alpha_{0,0}=2 c_{0}+g \gamma \tau$ is the incoming Riemann invariant in case of no motion, $\chi^{(0)}$ is given by Eq. 3.27), and $\tau_{0}=t-2 \omega$. By substituting Eq. 3.35) into Eq. (3.34), we obtain at first order in $\epsilon$ :

$$
\begin{aligned}
(2 \omega-t+\tau)\left(\chi_{t t}^{(1)}+2 \chi_{t \tau}^{(1)}\right)-\left(\chi_{\tau}^{(1)}-\chi_{t}^{(1)}-\alpha_{0,1}\right) & 3 \frac{3}{2}(2 \omega-t+\tau) \dot{\alpha}_{0,1}=0, \\
\chi_{t=\tau}^{(1)} & =0, \\
\chi_{\tau \tau}^{(0)}\left(t, \tau_{0}\right) \tau_{1}+\chi_{\tau}^{(1)}\left(t, \tau_{0}\right) & =0 .
\end{aligned}
$$

By letting $\Upsilon^{(1)}$ equal $\chi^{(1)}-(2 \omega-t+\tau) \alpha_{0,1} / 2$, we can rewrite Eq. 3.36a) as

$$
(2 \omega-t+\tau)\left(\Upsilon_{t t}^{(1)}+2 \Upsilon_{t \tau}^{(1)}\right)-\Upsilon_{\tau}^{(1)}+\Upsilon_{t}^{(1)}=0 .
$$

We then make the change of variables $\nu=-(2 \omega-t+\tau)$ and $\xi=\tau$, and Eq. (3.37) becomes

$$
\nu\left(2 \Upsilon_{\nu \xi}^{(1)}-\Upsilon_{\nu \nu}^{(1)}\right)-2 \Upsilon_{\nu}^{(1)}+\Upsilon_{\xi}^{(1)}=0
$$

Denoting the Fourier transform $\mathcal{F}(\cdot)$ with respect to $\xi$,

$$
\rho^{(1)}(\nu, s)=\mathcal{F}\left(\Upsilon^{(1)}(\nu, \xi)\right)(s)=\int_{-\infty}^{\infty} \Upsilon(\nu, \xi) e^{-i s \xi} \mathrm{d} \xi,
$$

we obtain from Eq. (3.38) a differential equation related to a Bessel equation:

$$
\nu\left(2 i s \rho_{\nu}^{(1)}-\rho_{\nu \nu}^{(1)}\right)-2 \rho_{\nu}^{(1)}+i s \rho^{(1)}=0,
$$

which has the general solution

$$
\rho^{(1)}(\nu, s)=e^{i s \nu}\left(A_{1}(s)\left[J_{0}(s \nu)-i J_{1}(s \nu)\right]+A_{2}(s)\left[i Y_{0}(s \nu)+Y_{1}(s \nu)\right]\right)
$$


with $J_{0,1}$ and $Y_{0,1}$ the Bessel functions of the first and second kinds. To recover $\Upsilon(\nu, \xi)$, we just need to take the inverse Fourier transform of Eq. (3.41), and by using $\Upsilon^{(1)}=\chi^{(1)}+\nu \alpha_{0,1} / 2$, we get

$$
\begin{aligned}
\chi^{(1)}(\nu, \xi)=\frac{1}{2 \pi} \int_{-\infty}^{\infty} e^{i s(\nu+\xi)} & \left(A_{1}(s)\left[J_{0}(s \nu)-i J_{1}(s \nu)\right]\right. \\
& \left.+A_{2}(s)\left[i Y_{0}(s \nu)+Y_{1}(s \nu)\right]\right) \mathrm{d} s-\frac{\nu}{2} \alpha_{0,1}
\end{aligned}
$$

with $\xi=\tau \leq t$.

\section{Boundary value assignment}

In order to calculate the unknown functions $A_{1}(s)$ and $A_{2}(s)$, we need to assign the boundary conditions (3.34). In $(\nu, \xi)$ space, $t=\tau$ corresponds to $\nu=-2 \omega$, and by imposing the first boundary condition, we get

$$
-\mathcal{F}\left(\alpha_{0,1}\right) \omega e^{2 i s \omega}=A_{1}(s)\left[J_{0}(2 s \omega)+i J_{1}(2 s \omega)\right]+A_{2}(s)\left[i Y_{0}(2 s \omega)-Y_{1}(2 s \omega)\right] .
$$

The second boundary condition is given by Eq. (3.36c), in which

$$
\begin{aligned}
\chi_{\tau}^{(1)}= & -\chi_{\nu}^{(1)}+\chi_{\xi}^{(1)} \\
= & \frac{i}{2 \pi} \int_{-\infty}^{\infty} e^{i s(\nu+\xi)}\left(A_{1}(s)\left[s J_{0}(s \nu)-i s J_{1}(s \nu)-\frac{J_{1}(s \nu)}{\nu}\right]\right. \\
& \left.\quad+A_{2}(s)\left[i s Y_{0}(s \nu)+s Y_{1}(s \nu)+\frac{Y_{1}(s \nu)}{i \nu}\right]\right) \mathrm{d} s+\frac{\alpha_{0,1}}{2}-\frac{\nu \dot{\alpha}_{0,1}}{2}
\end{aligned}
$$

is evaluated at $\tau=\tau_{0}$; i.e., $\nu=0$ needs to be finite. Evaluating Eq. (3.44) at $\nu=0$ gives us convergence when the coefficient $A_{2}(s)$ is zero, which avoids an unbounded result. Hence, from the first boundary condition (3.36b), coefficient $A_{1}(s)$ is given by

$$
A_{1}(s)=-\frac{\mathcal{F}\left(\alpha_{0,1}\right) \omega e^{2 i s \omega}}{J_{0}(2 s \omega)+i J_{1}(2 s \omega)} .
$$

The solution of incoming characteristic curves at first order is thus given by

$$
\chi^{(1)}(\nu, \xi)=-\frac{1}{2 \pi} \int_{-\infty}^{\infty} e^{i s(\nu+\xi+2 \omega)} \omega \mathcal{F}\left(\alpha_{0,1}\right) \frac{J_{0}(s \nu)-i J_{1}(s \nu)}{J_{0}(2 s \omega)+i J_{1}(2 s \omega)} \mathrm{d} s-\frac{\nu}{2} \alpha_{0,1} .
$$

The shoreline position must satisfy $\left.\chi_{\tau}\right|_{\tau=\tau_{\mathrm{s}}}=0$, and in the first-order approximation, it is given by

$$
x_{\mathrm{S}}(t)=\chi^{(0)}\left(t, \tau_{0}\right)+\epsilon\left[\chi_{\tau}^{(0)}\left(t, \tau_{0}\right) \tau_{1}+\chi^{(1)}\left(t, \tau_{0}\right)\right]+\mathcal{O}\left(\epsilon^{2}\right) .
$$


Since $\tau=\tau_{0}$ corresponds to $\nu=0$ and $\xi=t-2 \omega$, we get

$$
x_{\mathrm{s}}(t)=-\mathcal{F}^{-1}\left[\mathcal{F}\left(\alpha_{0,1}\right) \frac{\omega}{J_{0}(2 s \omega)+i J_{1}(2 s \omega)}\right] .
$$

\subsection{Effective Boundary Condition}

\subsubsection{Finite element implementation}

The region $x \in[B, L]$ will be approximated using a classical Galerkin finite element expansion. We use first-order spline polynomials on $N$ elements with $j=$ $1, \ldots, N+1$ nodes. The variational structure is simply preserved by substituting the expansions

$$
\breve{\phi}_{h}(x, t)=\phi_{j}(t) \varphi_{j}(x), \quad \breve{\psi}_{h}(x, t)=\psi_{j}(t) \varphi_{j}(x), \quad \text { and } \breve{\eta}_{h}(x, t)=\eta_{j}(t) \varphi_{j}(x)
$$

into Eq. (3.6) for $x \in[B, L]$ concerning $N$ elements and $(N+1)$ basis functions $\varphi_{j}$. We used the Einstein summation convention for repeated indices.

To ensure continuity and a unique determination, we employ Eq. (3.13) and substitute

$\phi(x, t)=\tilde{\phi}(x, t)+\phi_{1}(t) \varphi_{1}(x)+\frac{\tilde{\beta}}{h_{\mathrm{b}}} \psi_{1}(t) \varphi_{1}(x)$ and $\eta(x, t)=\tilde{\eta}(x, t)+\eta_{1}(t) \varphi_{1}(x)$

with $\varphi_{1}$ the basis function in element 0 for $x \in\left[x_{\mathrm{s}}, B\right]$, and with $\tilde{\phi}(B, t)=$ $\tilde{\eta}(B, t)=0$. For linear polynomials, the use of Eq. (3.49) in Eq. (3.6) yields

$$
\begin{aligned}
& 0=\delta \int_{0}^{T}\left[\mathbf{M}_{\mathrm{kl}} \phi_{k} \dot{\eta}_{l}-\frac{1}{2} g \mathbf{M}_{\mathrm{kl}} \eta_{k} \eta_{l}-\frac{1}{2} \mathbf{S}_{\mathrm{kl}} \phi_{k} \phi_{l}-\mathbf{B}_{\mathrm{kl}} \psi_{k} \phi_{l}-\frac{1}{2} \mathbf{A}_{\mathrm{kl}} \psi_{k} \psi_{l}-\frac{1}{2} \mathbf{G}_{\mathrm{kl}} \psi_{k} \psi_{l}\right. \\
& \left.+\int_{x_{\mathrm{s}}}^{B}\left(\phi \partial_{t} \eta-\frac{1}{2} g \eta^{2}-\frac{1}{2} h\left(\partial_{x} \phi\right)^{2}\right) \mathrm{d} x\right] \mathrm{d} t \\
& =\int_{0}^{T}\left[\left(\mathbf{M}_{\mathrm{kl}} \dot{\eta}_{l}-\mathbf{S}_{\mathrm{kl}} \phi_{l}-\mathbf{B}_{\mathrm{kl}} \psi_{l}\right) \delta \phi_{k}-\left(\mathbf{M}_{\mathrm{kl}} \dot{\phi}_{k}+g \mathbf{M}_{\mathrm{kl}} \eta_{k}\right) \delta \eta_{l}-\left(\mathbf{A}_{\mathrm{kl}} \psi_{l}+\mathbf{B}_{\mathrm{kl}} \phi_{l}+\mathbf{G}_{\mathrm{kl}} \psi_{l}\right) \delta \psi_{k}\right. \\
& +\int_{x_{\mathrm{s}}}^{B}\left(\left(\partial_{t} \eta+\partial_{x}\left(h \partial_{x} \phi\right)\right) \delta \tilde{\phi}-\left(\partial_{t} \phi+g \eta+\frac{1}{2} \partial_{x}^{2} \phi\right) \delta \tilde{\eta}\right) \mathrm{d} x+\left.(\phi \delta \eta)\right|_{x=x_{\mathrm{s}}} \frac{\mathrm{d} x_{\mathrm{s}}}{\mathrm{d} t}-\left.\left(\phi \partial_{t} \eta\right)\right|_{x=x_{\mathrm{s}}} \delta x_{\mathrm{s}} \\
& +\underline{\int_{x_{\mathrm{s}}}^{B}\left(\left(\partial_{t} \eta+\partial_{x}\left(h \partial_{x} \phi\right)\right) \varphi_{1} \delta \phi_{1}-\left(\partial_{t} \phi+g \eta+\frac{1}{2} \partial_{x}^{2} \phi\right) \varphi_{1} \delta \eta_{1}\right) \mathrm{d} x} \\
& \left.-\left.h \partial_{x} \phi\right|_{x=B} \delta \phi_{1}-\left.\frac{\tilde{\beta}}{h_{\mathrm{b}}} h \partial_{x} \phi\right|_{x=B} \delta \psi_{1}\right] \mathrm{d} t,
\end{aligned}
$$


where we introduced the mass and stiffness matrices $\mathbf{M}_{\mathrm{kl}}, \mathbf{S}_{\mathrm{kl}}, \mathbf{A}_{\mathrm{kl}}, \mathbf{B}_{\mathrm{kl}}$, and $\mathbf{G}_{\mathrm{kl}}$, and used endpoint conditions $\delta \eta_{k}(0)=\delta \eta_{k}(T)=0$, connection conditions $\delta \tilde{\eta}(B, t)=\delta \tilde{\phi}(B, t)=\delta \tilde{\psi}(B, t)=0$, and no-normal through-flow conditions at $x=L$. The matrices in Eq. 3.50 are defined as follows:

$$
\begin{aligned}
\mathbf{M}_{\mathrm{kl}} & =\int_{B}^{L} \varphi_{k} \varphi_{l} \mathrm{~d} x, \mathbf{S}_{\mathrm{kl}}=\int_{B}^{L} h \partial_{x} \varphi_{k} \partial_{x} \varphi_{l} \mathrm{~d} x, \mathbf{A}_{\mathrm{kl}}=\int_{B}^{L} \breve{\alpha} \partial_{x} \varphi_{k} \partial_{x} \varphi_{l} \mathrm{~d} x \\
\mathbf{B}_{\mathrm{kl}} & =\int_{B}^{L} \breve{\beta} \partial_{x} \varphi_{k} \partial_{x} \varphi_{l} \mathrm{~d} x, \text { and } \mathbf{G}_{\mathrm{kl}}=\int_{B}^{L} \breve{\gamma} \varphi_{k} \varphi_{l} \mathrm{~d} x
\end{aligned}
$$

Provided we let the size of the zeroth element go to zero such that the underlined terms in Eq. 3.50b vanish, the equations arising from Eq. (3.50) are

$$
\begin{array}{r}
\mathbf{M}_{\mathrm{kl}} \dot{\eta}_{l}-\mathbf{S}_{\mathrm{kl}} \phi_{l}-\mathbf{B}_{\mathrm{kl}} \psi_{l}-\left.\delta_{k 1}\left(h \partial_{x} \phi\right)\right|_{x=B^{-}}=0 \\
\mathbf{M}_{\mathrm{kl}} \dot{\phi}_{k}+g \mathbf{M}_{\mathrm{kl}} \eta_{k}=0 \\
\mathbf{A}_{\mathrm{kl}} \psi_{l}+\mathbf{B}_{\mathrm{kl}} \phi_{l}+\mathbf{G}_{\mathrm{kl}} \psi_{l}-\left.\delta_{k 1}\left(\frac{\tilde{\beta}}{h_{\mathrm{b}}} h \partial_{x} \phi\right)\right|_{x=B^{-}}=0
\end{array}
$$

with Kronecker delta symbol $\delta_{\mathrm{kl}}$ (one when $k=l$, and zero otherwise) and Eq. 3.10 for $x \in\left[x_{\mathrm{s}}, B\right]$ with boundary condition 3.12. Taking this limit does not jeopardize the time step, as this zeroth element lies in the continuum region, in which the resolution is infinite. The time integration is solved using ode45 in MATLAB, which uses its internal time step.

From Eq. 3.52, we note that we need the depth $h$ and the velocity $u$ from the nonlinear model at $x=B$, whose values are given at time $t=\tau$ in the characteristic space. The definitions (3.22), while using $\alpha=\alpha_{0}$ and $\beta$ in Eq. (3.30) with expansions up to first order, yield

$$
\begin{aligned}
h=c^{2} / g & =\frac{1}{16 g}\left(\alpha_{0}+\beta\right)^{2} \\
& =\left(\alpha_{0,0}+\chi_{t}^{(0)}-g \gamma t+\epsilon\left(\alpha_{0,1}+\chi_{t}^{(1)}\right)\right)^{2} / g \\
& =\left(\alpha_{0,0}+\frac{g \gamma t-\alpha_{0,0}}{2}-g \gamma t+\epsilon\left(\alpha_{0,1}+\chi_{t}^{(1)}\right)\right)^{2} / g \\
& =\left(c_{0}+\frac{1}{2} g \gamma(\tau-t)+\epsilon\left(\alpha_{0,1}+\chi_{t}^{(1)}\right)\right)^{2} / g \\
u=g \gamma t & +\frac{1}{2}\left(\beta-\alpha_{0}\right)=\epsilon\left(\alpha_{0,1}+2 \chi_{t}^{(1)}\right) .
\end{aligned}
$$

Note that for $\epsilon=0$, we indeed find the rest depth $h_{\mathrm{b}}(x)=\gamma x$. The function $\chi_{t}^{(1)}$ follows from evaluation of Eq. (3.46), and since $t=\tau$ is equivalent to $\nu=-2 \omega$, 
we immediately obtain

$$
\begin{aligned}
\left.\chi_{t}^{(1)}\right|_{t=\tau} & \equiv \chi_{\nu}^{(1)}(-2 \omega, \xi) \\
& =-\frac{i}{4 \pi} \int_{-\infty}^{\infty} e^{i s \xi} \mathcal{F}\left(\alpha_{0,1}\right) \frac{J_{1}(2 s \omega)}{J_{0}(2 s \omega)+i J_{1}(2 s \omega)} \mathrm{d} s-\frac{\alpha_{0,1}}{2} .
\end{aligned}
$$

The solutions of $h$ and $u$ at $t=\tau$ are thus given as follows:

$$
\begin{aligned}
h(B, t) & =h_{\mathrm{b}}+\eta \\
& =\frac{c_{0}^{2}}{g}+\epsilon \frac{c_{0}}{g} \mathcal{F}^{-1}\left[\mathcal{F}\left(\alpha_{0,1}\right) \frac{J_{0}(2 s \omega)}{J_{0}(2 s \omega)+i J_{1}(2 s \omega)}\right] \\
u(B, t) & =-\epsilon \mathcal{F}^{-1}\left[\mathcal{F}\left(\alpha_{0,1}\right) \frac{i J_{1}(2 s \omega)}{J_{0}(2 s \omega)+i J_{1}(2 s \omega)}\right] .
\end{aligned}
$$

In order to calculate the solutions for $h$ and $u$ at $x=B$ and the shoreline position, we need the data of incoming Riemann invariants at the first order as follows:

$$
\begin{aligned}
\epsilon \alpha_{0,1} & \approx \alpha-\alpha_{0,0} \\
& =\left.2(\sqrt{g(\gamma B+\breve{\eta})}-\sqrt{g \gamma B})\right|_{x=B^{+}}-\left.\breve{u}\right|_{x=B^{+}},
\end{aligned}
$$

which is obtained by disregarding higher-order terms in Eq. (3.35a). This expression is actually the incoming Riemann invariant in LSWE Kristina et al., 2012. By imposing the effective boundary condition (EBC) and choosing the location $x=B$ before the nonlinearity arises, we thus do actually solve for the perturbation expansion in the nonlinear area, but we do not perturb the incoming wave data.

The values $\breve{\eta}$ and $\breve{u}$ in Eq. (3.56) are obtained from the simulation area $[B, L]$. In this region, we only have the values of $\breve{\eta}, \breve{\phi}$, and $\breve{\psi}$. The depth-averaged velocity $u\left(B^{+}, t\right)$ is determined by using the approximation 3.13 as follows:

$$
\breve{u}=\partial_{x} \breve{\phi}+\frac{\breve{\beta}}{h_{\mathrm{b}}} \partial_{x} \breve{\psi} \text { at } x=B^{+},
$$

which is the limit from the right at node 1.

The solutions of $\eta=h-h_{\mathrm{b}}$ and $u$ in Eq. (3.55) account for the reflected wave, so we may define

$$
\eta=\eta^{\mathrm{I}}+\eta^{\mathrm{R}} \text { and } u=u^{\mathrm{I}}+u^{\mathrm{R}},
$$

where $\eta^{\mathrm{I}}$ and $\eta^{\mathrm{R}}$ are the wave elevations of incoming and reflected waves, respectively, at $x=B$. This superposition is also described in Antuono and Brocchini 
(2007, 2010), and is actually in line with our EBC concept, since linearity holds in the simulation area. To obtain the expression for the reflected wave, we need to know the incoming one. Using the knowledge of incoming and outgoing Riemann invariants in the LSWE as derived in Kristina et al. [2012], the observation operator is given by

$$
\mathcal{O}=h \breve{u}+c \breve{\eta}=2 c \eta^{\mathrm{I}}
$$

which is calculated using approximation (3.57). We can thus calculate the incoming wave elevation for any given wave signal at $x=B$. Implementation of this observation operator allows us to have any initial waveform at the point of tsunami generation, and to let it travel over the real bathymetry to the seaward boundary point $x=B$. From Eq. (3.55), the expressions for the reflected wave are as follows:

$$
\begin{aligned}
\eta^{\mathrm{R}} & =\mathcal{M}\left(\eta^{\mathrm{I}}\right)=\frac{c_{0}}{g} \mathcal{F}^{-1}\left[\mathcal{F}\left(\epsilon \alpha_{0,1}\right) \frac{J_{0}(2 s \omega)}{J_{0}(2 s \omega)+i J_{1}(2 s \omega)}\right]-\eta^{\mathrm{I}} \\
u^{\mathrm{R}} & =\mathcal{M}\left(u^{\mathrm{I}}\right)=-\mathcal{F}^{-1}\left[\mathcal{F}\left(\epsilon \alpha_{0,1}\right) \frac{i J_{1}(2 s \omega)}{J_{0}(2 s \omega)+i J_{1}(2 s \omega)}\right]-u^{\mathrm{I}},
\end{aligned}
$$

where the Fourier transform and its inverse for any incoming wave signal are evaluated using the FFT and IFFT functions in MATLAB.

The influxing operator is defined as the coupling condition in Eq. (3.52) to send NSWE result to the simulation area. It is shown that we need the value of $h \partial_{x} \phi$, and hence

$$
\mathcal{I}=h \partial_{x} \phi=\left(h_{\mathrm{b}}+\eta\right) u
$$

In order to verify the EBC implementation, we perform numerical simulations with a code that couples the LSWE in the simulation area to the NSWE in the model area Bokhove, 2005, Klaver, 2009]. For the numerical simulation of the LSWE, we use a finite element method, while for the NSWE, we use a finite volume method. The implementation of the finite volume method is explained in Appendix 3.6.

\subsection{Study Case}

Three test cases are considered. The first one is a synthetic one concerning a solitary wave, such that we can compare it with other results. Subsequently, we consider periodic wave influx as the second case to test the robustness of the technique when there is continuous interaction between the incoming and reflected 
waves. The third case is a more realistic one concerning tsunami propagation and run-up based on a simplified bathymetry at the Aceh coastline.

The location of the EBC point is determined from the linearity condition $\epsilon=A_{0} / h_{0} \ll 1$. From linear theory, the wave amplification over depth is given by the ratio $A_{0}=A \sqrt[4]{h / h_{0}}$, where $A$ and $h$ are the initial wave amplitude and depth. Hence, the EBC point must be located at a depth

$$
h_{0} \gg \sqrt[5]{A^{4} h / \epsilon^{4}} .
$$

Since a dispersive model is also used in the simulation area, we will discuss the dispersion effect at this EBC point as well. The non-dispersive condition is given by $\mu^{2}=\left(k_{0} h_{0}\right)^{2} \ll 1$, where $k_{0}=2 \pi / \lambda_{0}$ is the wavenumber and $\lambda$ is the wavelength. In linear wave theory, the wavelength decreases with the square root of the depth when running in shallower water, that is $\lambda_{0}=\lambda \sqrt{h_{0} / h}$. Using this relation, we can thus investigate the significance of the dispersion given the information of the initial condition and bathymetry profile.

\subsubsection{Solitary wave}

The run-up of a solitary wave is studied by means of the well-known case of Synolakis 1987. A solitary wave centered at $x=x_{0}$ at $t=0$ has the following surface profile:

$$
\eta(x, 0)=A \operatorname{sech}^{2} \kappa\left(x-x_{0}\right) .
$$

We benchmark the EBC implementation and the coupling of numerical solutions to experimental data of Synolakis [1987 provided at the NOAA Center for Tsunami Research (http://nctr.pmel.noaa.gov/benchmark/). Solitary wave run-up over a canonical bathymetry is considered with the scaled amplitude $A=0.0185$ and $\kappa=\sqrt{3 A / 4}=0.1178$. The initial condition is centered at $x_{0}=37.35$ over the bathymetry, with a constant slope $\gamma=1 / 19.85$ for $x<19.85$. The EBC point is located at $x=10$, such that the domain is divided into the model area for $x \in[-5,10]$ and the simulation area for $x \in[10,80]$. The spatial grid sizes are $\Delta x=0.25$ in the simulation area and $\Delta x=0.0125$ in the model area for the numerical solution of NSWE. In all cases, several spatial resolutions have been applied to verify numerical convergence. For the time integration, we use the fourth-order ode45 solver that estimates its own time step in MATLAB.

The simulations with the EBC implementation and the coupling of the numerical solutions are only presented for the LSWE model in the simulation area since the initial condition has a long wavelength, and thus dispersion effects will not appear. Figure 3.3 shows the time evolution of this profile for the scaled time $t=30-70$ with 10 increments. It can be seen that the EBC implementation 
(a)

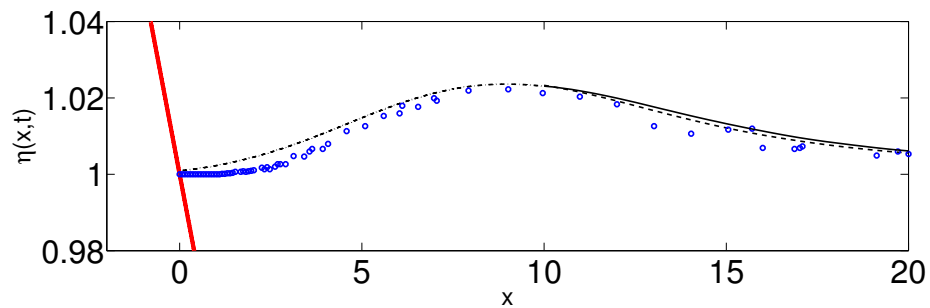

(b)

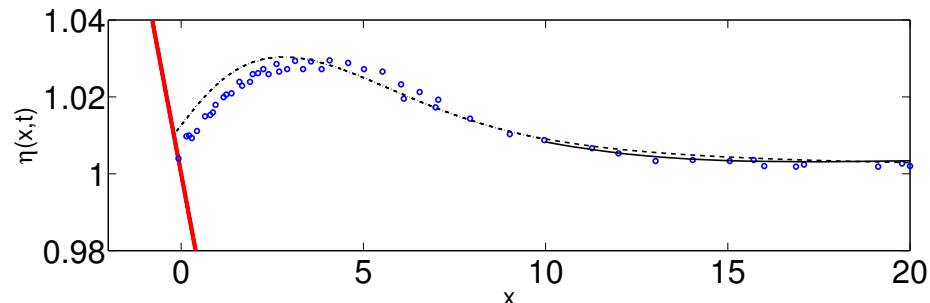

(c)

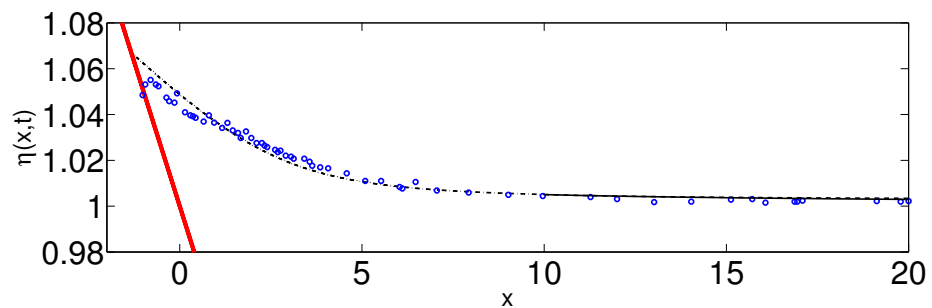

(d)

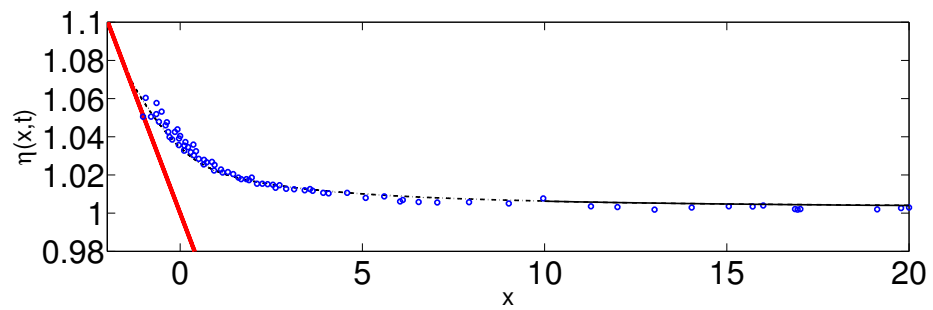

(e)

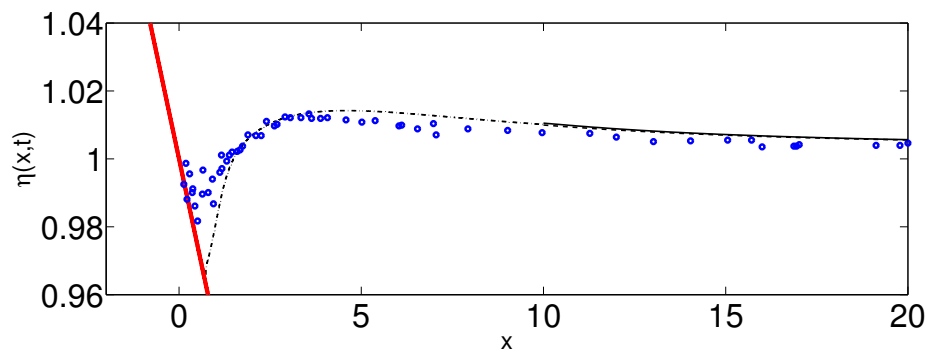

Figure 3.3: Run-up of a solitary wave over a canonical bathymetry at times (a) $t=$ 30 , (b) $t=40$, (c) $t=50$, (d) $t=60$, (e) $t=70$. The solid line is LSWE with $E B C$ implementation at $x=10$, the dashed and dotted-dashed lines are the couplings of the LSWE and NSWE models respectively, and the symbols are the laboratory data of Synolakis [1987]. 


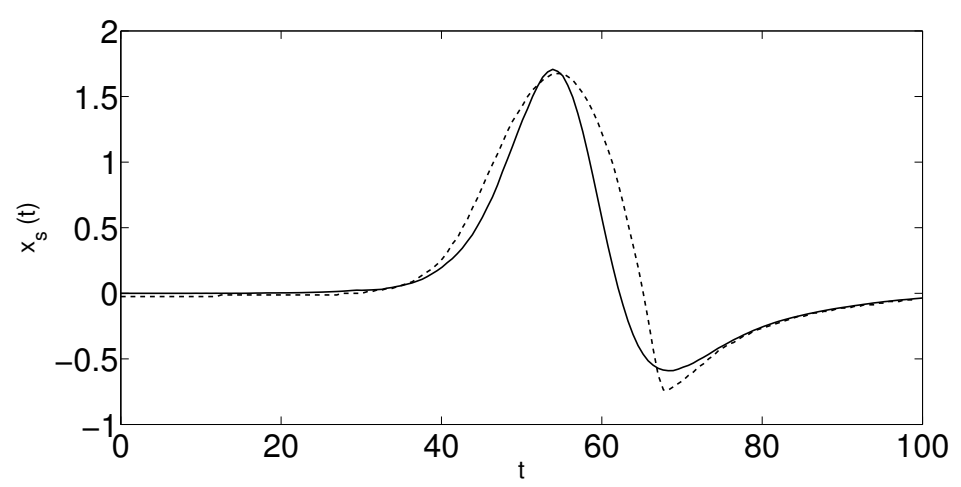

Figure 3.4: The shoreline movement of a solitary wave introduced in Synolakis [1987]. The LSWE model coupled to the NSWE is shown by the dashed line, while the solid one is the shoreline movement of the LSWE model with an EBC implementation.

and the coupling of numerical solutions agree well with the laboratory data. The comparison of the shoreline movement between the simulation with the EBC implementation and the coupling of numerical solutions is shown in Fig. 3.4. For the simulation till the scaled physical time $t=100$, the computational time for the coupled numerical solutions in both domains is $10.9 \mathrm{~s}$, while the computational time of the simulation with the EBC implementation only takes about $18 \%$ of that time. Hence, we notice that the simulation with the EBC reduces the computational time significantly, up to approximately $82 \%$, compared with the computational time in the whole domain.

In order to show the dispersion effect, we consider a shorter wave with the profile given in Eq. (3.63) for $\kappa=0.04, x_{0}=150 \mathrm{~m}$, and $A=0.1 \mathrm{~m}$. The bathymetry is given by a constant depth of $10 \mathrm{~m}$ for $x>50 \mathrm{~m}$, continued by a constant slope $\gamma=1 / 5$ towards the shore. A uniform spatial grid $\Delta x=1 \mathrm{~m}$ is used in the simulation area, and $\Delta x$ equals $0.015 \mathrm{~m}$ in the model area for the numerical solution of the NSWE. Evaluating Eq. (3.62) for $\epsilon=0.02 \ll 1$, the EBC point must be located at $h_{0} \gg 3.3 \mathrm{~m}$. Accordingly, we choose this seaward boundary point at $h_{0}=10 \mathrm{~m}$ at the toe of the slope, that is at $x=B=50 \mathrm{~m}$. We therefore divide the domain into the simulation area for $x \in[50,250] \mathrm{m}$, and the model area for $x \in[-5,50] \mathrm{m}$.

In Fig. 3.5, we can see the initial profile of the solitary wave. The dashed and dotted-dashed lines represent the coupling of the linear model (LSWE or LVBM) with the NSWE, respectively, and the solid one represents the linear model with an EBC implementation. The thick solid line is the sloping bathymetry. Comparisons between these two simulations at several time steps can be seen in Fig. 3.6 (left: LSWE; right: LVBM). Comparing the left and right figures, we can see that 


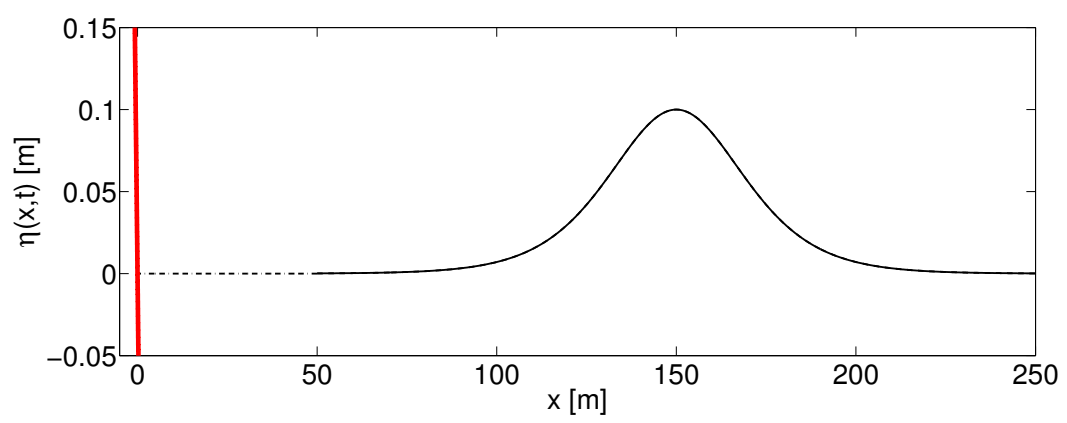

Figure 3.5: A solitary wave initial condition for the NSWE (dotted-dashed line) coupled to the linear model (dashed line), and the linear model with the EBC implementation (solid line) at $x=50 \mathrm{~m}$. The solid and dashed lines are on top of one another.

the wave is slightly dispersed in the LVBM. Because we have a flat bathymetry in this case, the dispersion ratio in the simulation area is constant and given by $\mu^{2}=0.39<1$. Hence, it is shown that the long waves propagate faster than the shorter ones in LVBM simulations. In Fig. 3.7, the shoreline movement caused by this solitary wave is shown with the dashed line for the coupled numerical simulation, and the solid one for linear model with an EBC implementation. The paths of characteristic curves forming the shoreline are also shown in this figure. We can see that the shoreline is formed by the envelope of the characteristic curves. The result with the LVBM shows a lower run-up but a higher run-down, with some oscillations at later times.

For a simulation till physical time $t=40 \mathrm{~s}$, the computational time for the coupled numerical solutions in both domains is 3.3 times the physical time for the LSWE and 2.2 times the physical time for the LVBM, while the computational time of the simulation using an EBC only takes 0.12 times the physical time for the LSWE and 0.06 times that for the LVBM. Hence, we notice that the simulation with the EBC reduces the computational time significantly, up to approximately $97 \%$, compared with the computational time for the numerical models in the entire domain. The computational time for the LSWE with an EBC is slower than the one with LVBM and an EBC, because the internal time step of the ode45 time step routine in MATLAB required a smaller time step $\Delta t$ (compared to the LVBM) to preserve stability.

The shoreline movement of our result compares well with the one of Choi et al. 2011. We can see the comparison in Fig. 3.8. The solution of Choi et al. 2011] gives a higher prediction for the shoreline, but it cannot follow the subsequent small positive wave. It may be caused by neglecting the reflection wave and nonlinear effects in their formulation. We also compare the free-surface profile 
(a)
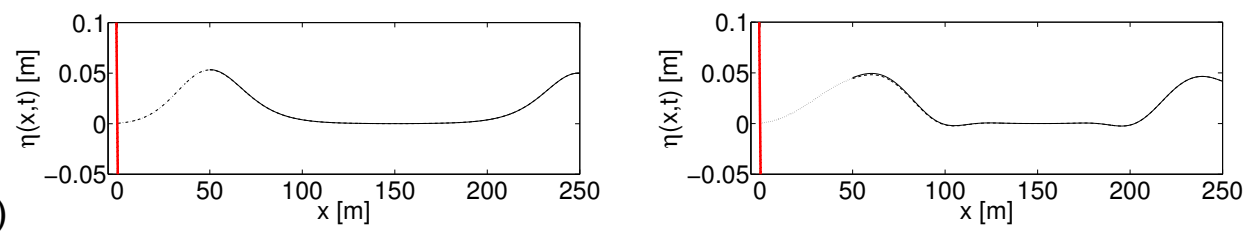

(b)
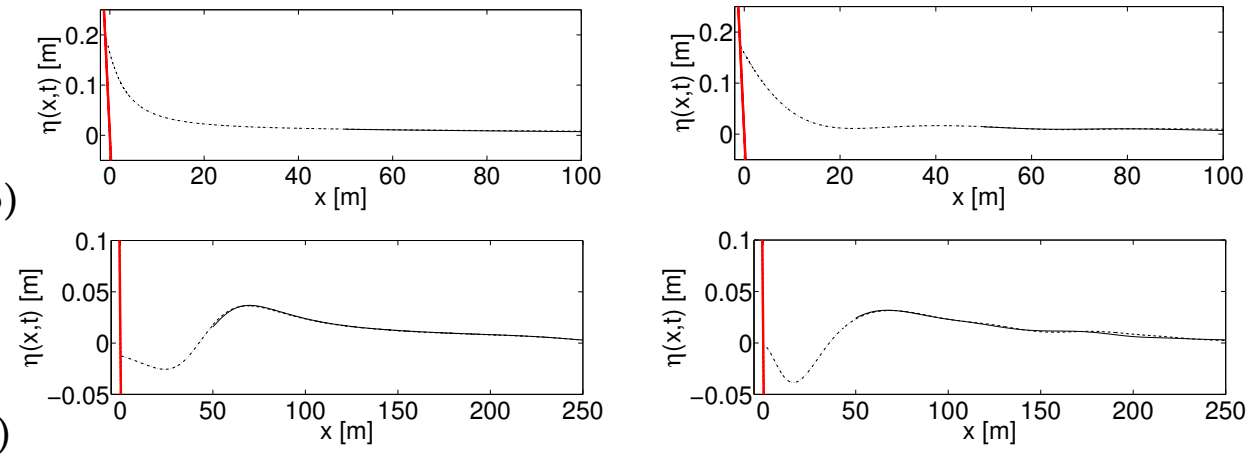

(c)
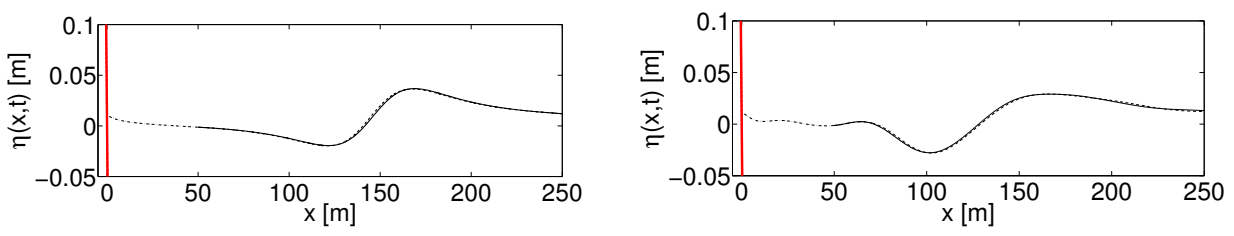

Figure 3.6: Free-surface profiles of solitary wave propagation are shown for the coupled linear model (left: LSWE; right: LVBM) with the NSWE (dashed and dotted-dashed lines), and for the linear model with an EBC implementation (solid line), at times (a) $t=$ $10 \mathrm{~s}$, (b) $t=20 \mathrm{~s},(\boldsymbol{c}) t=30 \mathrm{~s}$, and (d) $t=40 \mathrm{~s}$. The solid and dashed lines are on top of one another.

(a)

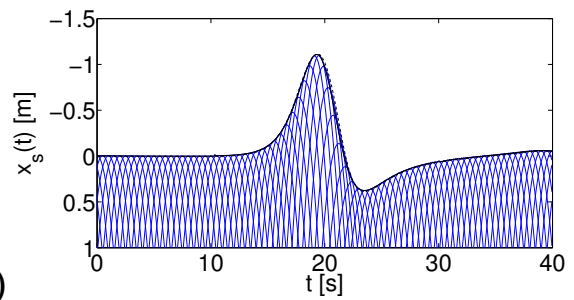

(b)

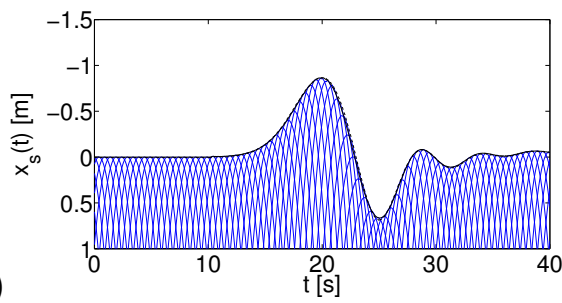

Figure 3.7: The shoreline movement of a solitary wave for the linear model (a: LSWE; b: LVBM) coupled to the NSWE (dashed line), and the linear model with an EBC implementation (solid line). Paths of the first-order characteristic curves are shown by the thin lines. 
for several time steps in Fig. 3.9. The implementation of the hard-wall boundary condition at $x=B$ in the method of Choi et al. 2011] causes inaccuracies in the prediction of the point-wise wave height in the entire domain. In this case, the effect of reflected waves for shoreline movement prediction is small, but it may become important when a compound of waves arrives at the coastline.

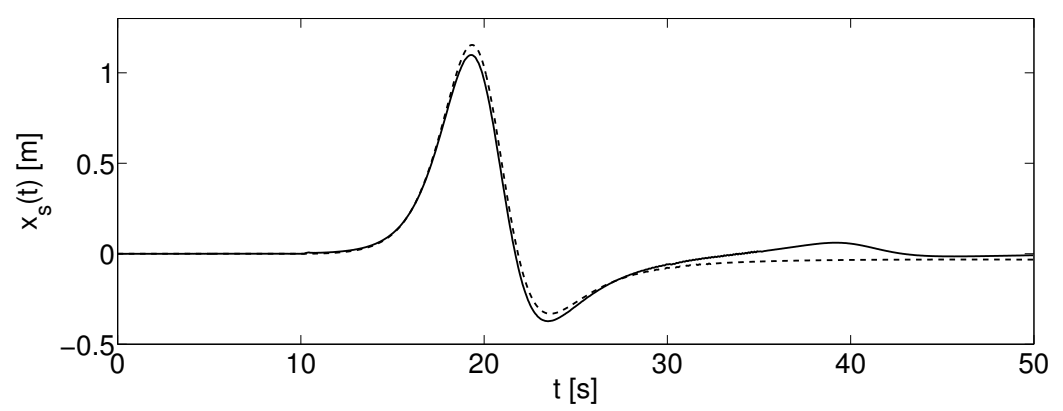

Figure 3.8: Comparison of the shoreline movement of Choi et al. [2011] (dashed line) and the LSWE with an EBC simulation (solid line) for the solitary wave case.

(a)

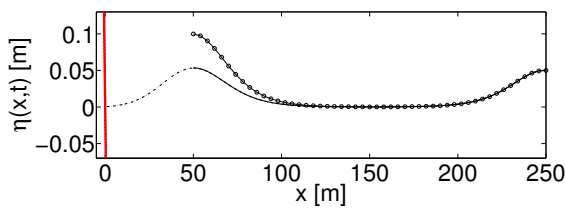

(c)

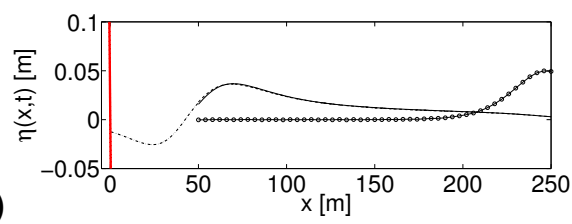

(b)

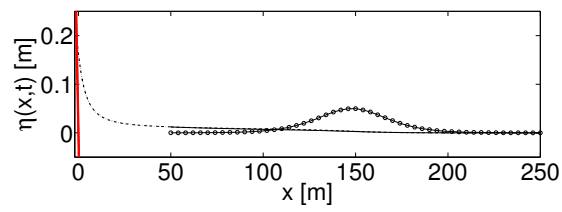

(d)

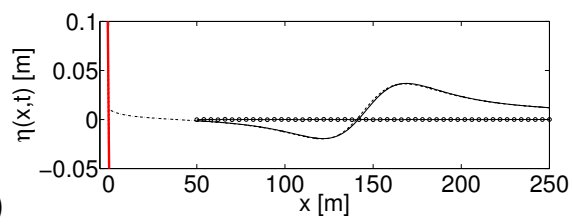

Figure 3.9: Free-surface profiles of solitary wave propagation are shown for the coupled $L S W E$ with the NSWE (dashed and dotted-dashed lines), for the LSWE with an EBC implementation (solid line), and for the LSWE with Choi's method (solid line with the ' $O$ ' marker) at times (a) $t=10 \mathrm{~s}$, (b) $t=20 \mathrm{~s}$, (c) $t=30 \mathrm{~s}$, and (d) $t=40 \mathrm{~s}$. The solid and dashed lines are on top of one another.

\subsubsection{Periodic wave}

Using the same bathymetry profile as in the previous case, we influx a periodic wave at the right boundary $(x=L)$ with the profile

$$
\eta(L, t)=A \sin (2 \pi t / T)
$$


in which $A=0.05 \mathrm{~m}$ is the amplitude and $T=20 \mathrm{~s}$ is the period. A smoothened characteristic function until $t=10 \mathrm{~s}$ is used in influxing this periodic wave. We use a uniform spatial grid $\Delta x=1 \mathrm{~m}$ in the simulation area and $\Delta x=0.015 \mathrm{~m}$ in the model area for the numerical solution of the NSWE.

(a)
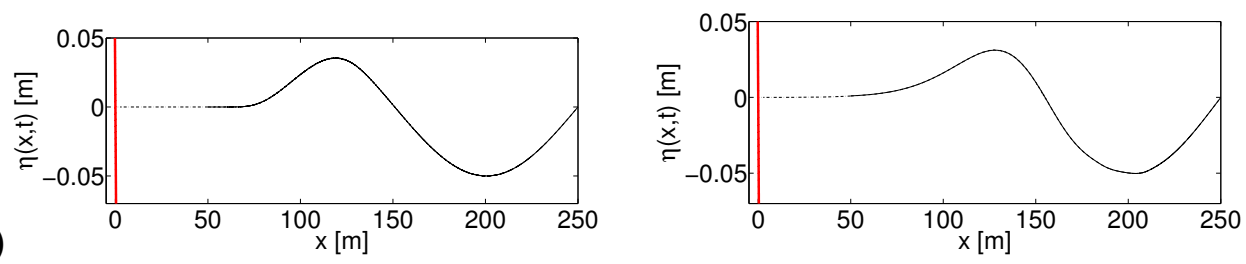

(b)
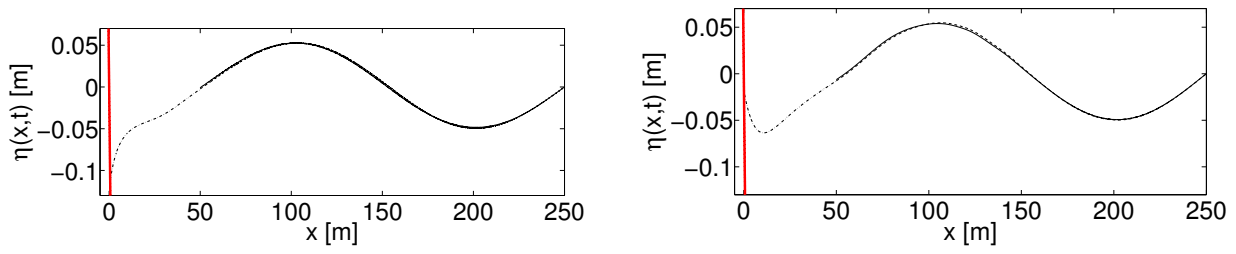

(c)
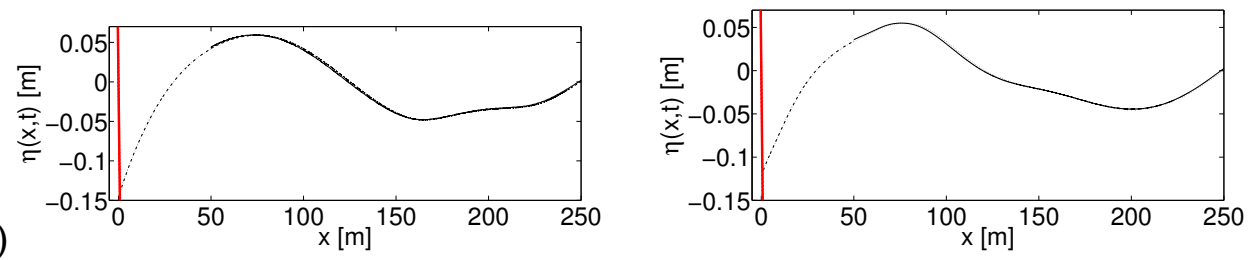

(d)
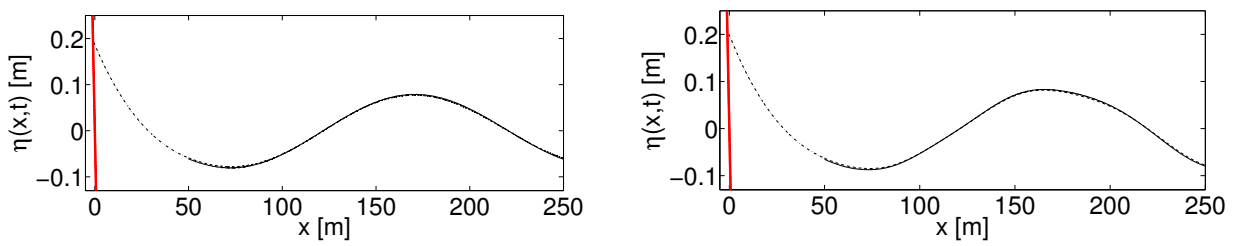

Figure 3.10: Free-surface profiles of periodic waves are shown for the coupled linear model (left: LSWE; right: LVBM) with the NSWE (dashed and dotted-dashed lines), and for the linear model with an EBC implementation (solid line), at times (a) $t=20 \mathrm{~s}$, (b) $t=40 \mathrm{~s},(\boldsymbol{c}) t=60 \mathrm{~s}$, and (d) $t=75 \mathrm{~s}$. The solid and dashed lines are on top of one another in several plots.

As in the previous case, we also choose the seaward boundary point at $h_{0}=$ $10 \mathrm{~m}$ at the toe of the slope, that is, at $x=B=50 \mathrm{~m}$. The simulation area is thus for $x \in[50,250] \mathrm{m}$ and the model area is for $x \in[-5,50] \mathrm{m}$. Comparisons between these two simulations at several time steps can be seen in Fig. 3.10 (left: LSWE; right: LVBM). We can see in the comparison that the wave is slightly dispersed in the LVBM. The dispersion ratio in the simulation area is given by $\mu^{2}=0.0986<1$, which is less dispersive than the previous case. In 
(a)

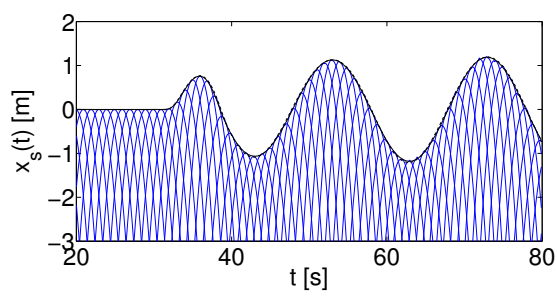

(b)

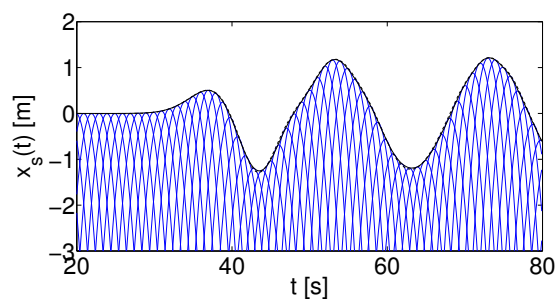

Figure 3.11: The shoreline movement of periodic waves for the linear model (a: LSWE; $\boldsymbol{b}$ : LVBM) coupled to the NSWE (dashed line), and for the linear model with an EBC implementation (solid line). Paths of the first-order characteristic curves are shown by the thin lines.

Fig. 3.11, the shoreline movement caused by the periodic wave as well as the paths of characteristic curves forming the shoreline are shown. Observing the results of this case, we can conclude that the EBC technique can deal robustly with consecutive interactions between incoming and reflected waves.

For a simulation till the physical time $t=80 \mathrm{~s}$, the computational time for the coupled numerical solutions in both domains is 1.83 times the physical time for the LSWE, and 2.01 times the physical time for the LVBM, while the computational time of the simulation using an EBC only takes 0.07 times the physical time of the LSWE and 0.06 times that of the LVBM. Obviously, we notice that the simulation with the EBC reduces the computational time up to approximately $97 \%$, compared with the computational time for whole domain simulation.

As mentioned in the Introduction, a resonant phenomena were investigated by Stefanakis et al. 2011] for monochromatic waves on a plane beach. Subsequently, Ezersky et al. [2013] used three piece-wise linear profiles of unperturbed depths (see Fig. 3.12 , akin to a real coastal bottom topography, to find the analytical run-up amplification due to resonance effects. We follow this bathymetry profile, with $\tan \alpha=0.0036, \tan \beta=0.0414, h_{0}=2500 \mathrm{~m}$, and $h_{1}=200 \mathrm{~m}$. These choices roughly characterize the Indian coast bathymetry [Neetu et al., 2011]. The EBC point is located at the edge of the last beach slope. We influx a periodic wave (3.64) with amplitude $A=1 \mathrm{~m}$ and $\omega=2 \pi / T=0.0009 \mathrm{rad} / \mathrm{s}$. As a result, we get 10.67 times amplification, as shown in the run-up height $R(t)$ in Fig. 3.13, while the result of Ezersky et al. 2013] gives about 12 times amplification. It should be noted that they use a linear approximation to calculate the amplification of periodic waves. In our results, the NSWE model is employed in the last beach slope region. The period of this wave is approximately $2 \mathrm{~h}$, and it coincides with the observed tsunami at the Makran coast, according to Neetu et al. [2011. In nature, one would not expect a tsunami of a monochromatic wave train. The investigation of Stefanakis et al. 2011 for the October 25, 2010 Mentawai Islands 
tsunami showed that the period of the dominant mode of the incident wave is within the resonant regime, and it explained the fact that the highest run-up is not driven by the leading and highest waves.

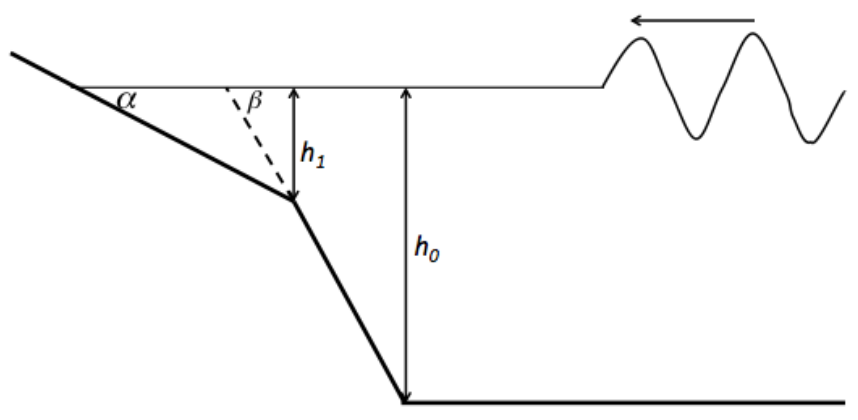

Figure 3.12: The three piece-wise linear bathymetry profiles.

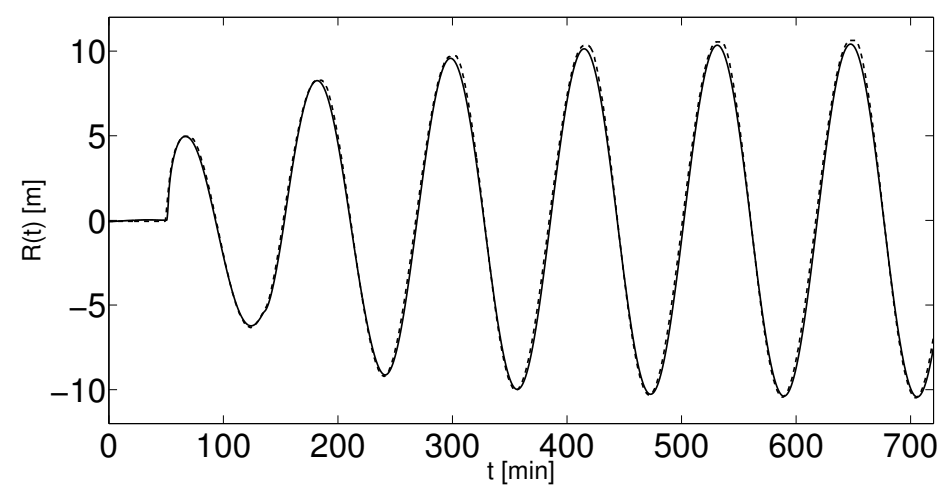

Figure 3.13: The run-up height of periodic waves with initial amplitude $A=1 \mathrm{~m}$ and frequency $\omega=0.0009 \mathrm{rad} / \mathrm{s}$. The solid line is the run-up height calculated by employing the LSWE model in the simulation area with the EBC implementation. The dashed one is the result of coupling the NSWE model in the model area to the LSWE model in the simulation area.

\subsubsection{Simulation using simplified Aceh bathymetry}

The bathymetry near Aceh, Indonesia, is displayed in Fig. 3.14. Figure 3.14a concerns bathymetry data from GEBCO (General Bathymetric Chart of the Oceans), with a zero value on land. Figure $3.14 \mathrm{~b}$ concerns the cross-section at $\left(95.0278^{\circ} \mathrm{E}, 3.2335^{\circ} \mathrm{N}\right)-\left(96.6583^{\circ} \mathrm{E}, 3.6959^{\circ} \mathrm{N}\right)$ shown by the solid line. The 2004 Indian Ocean tsunami was a result of an earthquake of magnitude of Mw 9.1 
at the epicenter point $\left(95.854^{\circ} \mathrm{E}, 3.316^{\circ} \mathrm{N}\right)$, shown by the symbol in Figure 3.14 a. Presently, we consider the following initial $N$-wave profile

$$
\begin{gathered}
\eta(x, 0)=A f(x) / S \text { with } f(x)=\frac{\mathrm{d}}{\mathrm{d} x} \exp \left(-\left(x-x_{0}\right)^{2} / w_{0}{ }^{2}\right) \\
\text { and } S=\max (f(x)),
\end{gathered}
$$

and where the initial velocity potential is zero. We take $A=0.4 \mathrm{~m}$, the position of the wave profile $x_{0}=107.4 \mathrm{~km}$, and a width $w_{0}=35 \mathrm{~km}$.

(a)

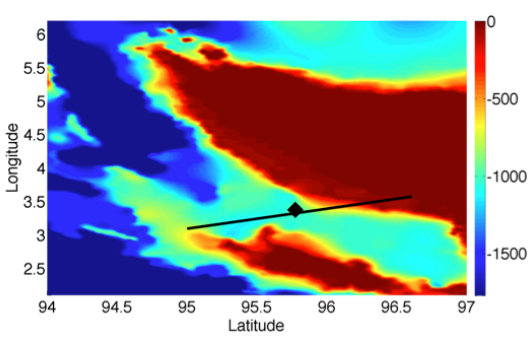

(b)

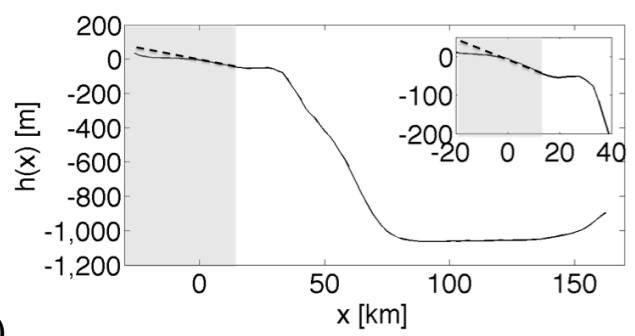

Figure 3.14: Bathymetry near Aceh (a) and the cross-section (b) at $\left(95.0278^{\circ} \mathrm{E}\right.$, $\left.3.2335^{\circ} \mathrm{N}\right)-\left(96.6583^{\circ} \mathrm{E}, 3.6959^{\circ} \mathrm{N}\right)$. The solid line concerns the bathymetry data, and the dashed line concerns the approximation used in the simulations.

The location of the EBC point is also determined from Eq. (3.62). For $\epsilon=$ $0.02 \ll 1$, the linear model is valid for $h_{0} \gg 25.1 \mathrm{~m}$. Hence, we choose the EBC point at depth $h_{0}=41.4 \mathrm{~m}$, which is located at $x=B=12.4 \mathrm{~km}$. The simulation area is thus for $x \in[12.4,162.4] \mathrm{km}$, where we follow the real bathymetry of Aceh to calculate the wave propagation. It is coupled to the model area for $x \in[-8.6,12.4] \mathrm{km}$, where a uniform slope with gradient $\gamma=1 / 300$ is used to calculate the reflection and shoreline position. We use a non-uniform grid according to the depth, with ratio $\sqrt{h_{0} / h}$ as the decrease in the wavelength when traveling from a deep region with depth $h$ and a shallower region with depth $h_{0}$ in linear wave theory. The grid size used in the simulation area is $\Delta x=305 \mathrm{~m}$ at the shallowest point near $x=B$. This choice of spatial resolution is fairly close to other numerical tsunami simulations (Horrillo et al., 2006 use $\Delta x=100 \mathrm{~m}$ offshore and $\Delta x=10 \mathrm{~m}$ onshore in one-dimensional (1D) simulations). For the numerical solution of the NSWE in the model area, a uniform grid with $\Delta x=3 \mathrm{~m}$ is used.

In Fig. 3.15, we show the initial profile. Comparisons between these two simulations at several time steps can be seen in Fig. 3.16. In this case, the wave elevation measured at $B$ has changed from its initial condition due to the reflection from the bathymetry before entering the model area; see Fig. 3.16a and b. We hardly see any differences between the LSWE and LVBM simulations 


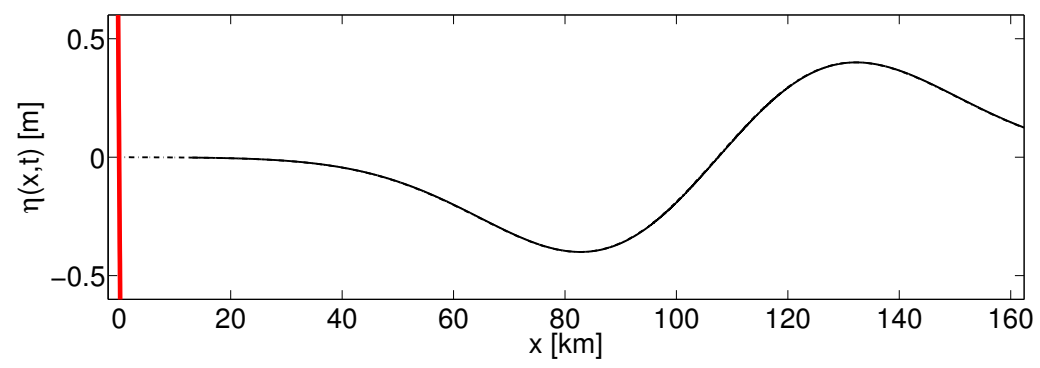

Figure 3.15: The initial condition for the Aceh case is shown for the linear model coupled to the NSWE (dashed and dotted-dashed lines) an for the linear model with an $E B C$ implementation (solid line). The solid and dashed lines are on top of one another.

because the wavelength is much greater than the depth. The dispersion ratio at the initial condition is given by $\mu^{2}=0.002 \ll 1$, and at the EBC point, it is approximately $\mu^{2}=7.5 \times 10^{-5} \ll 1$. The dispersion effect is therefore not significant in this case. In Fig. 3.17, the shoreline position is shown with the dashed line for the coupled numerical simulation, and the solid one for the linear model with the EBC implementation. Paths of the characteristic curves are also shown in this figure. From this plot, it is shown that the wave runs up $1 \mathrm{~km}$ in the horizontal direction in approximately $10 \mathrm{~min}$, roughly in the time interval from 50 to $60 \mathrm{~min}$. For the given slope, it corresponds to a $3.3 \mathrm{~m}$ run-up height.

For the simulation till the physical time $t=120 \mathrm{~min}$, the computational time for the coupled numerical solutions in both domains is 0.03 times the physical time for the LSWE and 0.03 times the physical time for the LVBM, while the computational time of the simulation using an EBC only takes 0.003 times the physical time for the LSWE and 0.004 times that for the LVBM. We again notice that the simulations using the EBC reduce the computational times up to approximately $92 \%$ of the computational times with the coupled model in the entire domain. In this case, the simulation with the LSWE is faster, as expected, since the LVBM involves more calculations within the same time step.

For the case when breaking occurs, we use the same profile, with an amplitude twice as high $(A=0.8 \mathrm{~m})$. In Fig. 3.18, the shoreline position is presented. Compared to the numerical NSWE solution, it can be seen that the shoreline movement is well represented by the characteristic curves, while the shoreline position $x_{\mathrm{s}}(t)$ given by Eq. (3.48) gives a less accurate result. Breaking occurs when two incoming characteristic curves intersect before reaching the shoreline. As can be seen in the right figure, the first breaking takes place at approximately $t=45 \mathrm{~min}$. The corresponding free-surface profiles at several times before and after the breaking are shown in Fig. 3.19. 
(a)

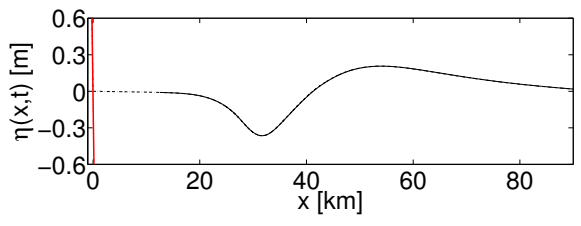

(b)

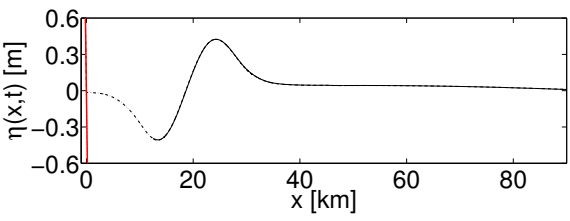

(c)

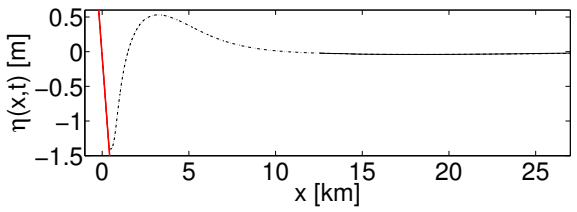

(d)

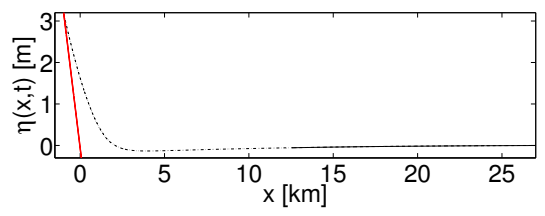

(e)

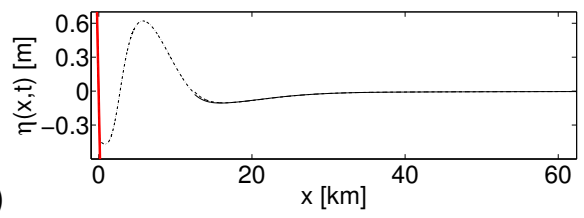

(f)

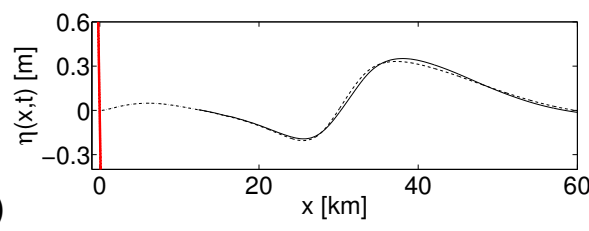

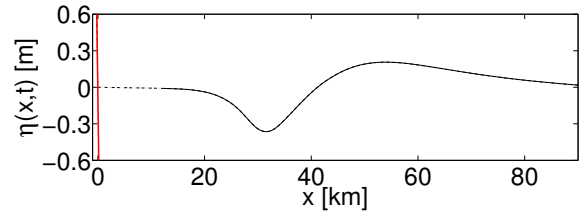
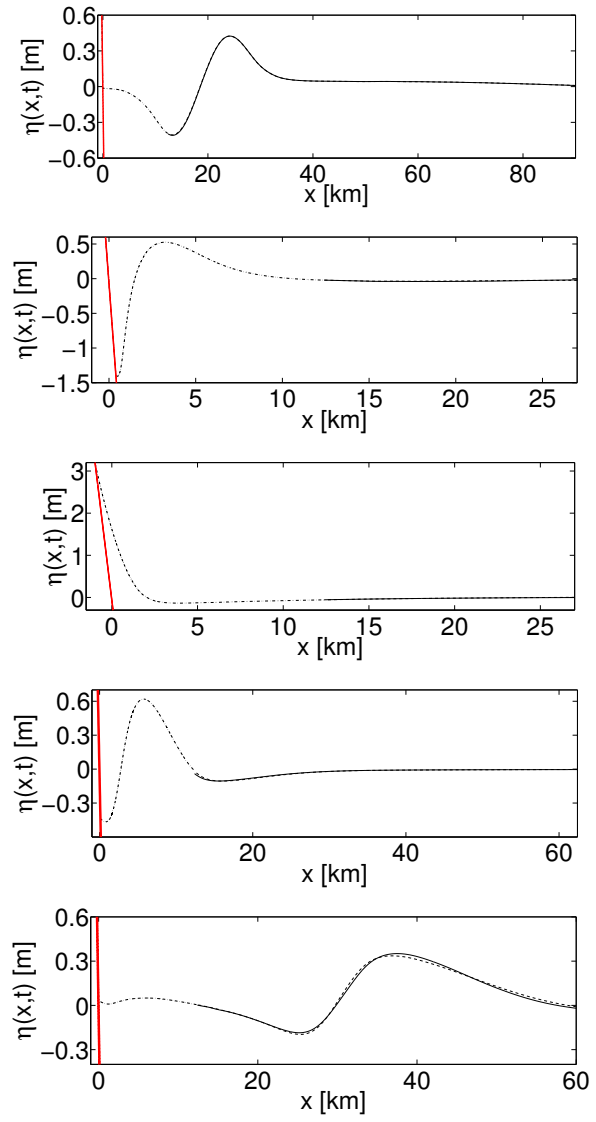

Figure 3.16: Free-surface profiles of Aceh simulations with the linear model (left: $L S W E$; right: LVBM) coupled to the NSWE are shown by the dashed and dotted-dashed lines, and for simulations of a linear model with an EBC implementation, are shown by the solid line at times (a) $t=800 \mathrm{~s}$, (b) $1600 \mathrm{~s}$, (c) $2700 \mathrm{~s}$, (d) $3200 \mathrm{~s}$, (e) $4000 \mathrm{~s}$, and (f) $5400 \mathrm{~s}$. The solid and dashed lines are on top of one another. 
(a)

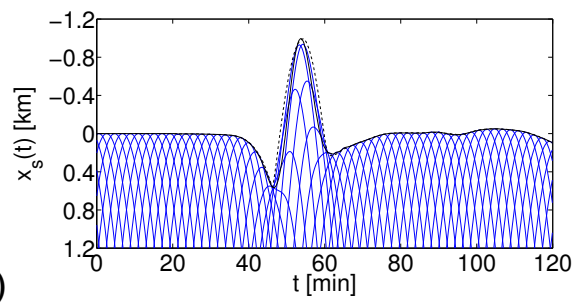

(b)

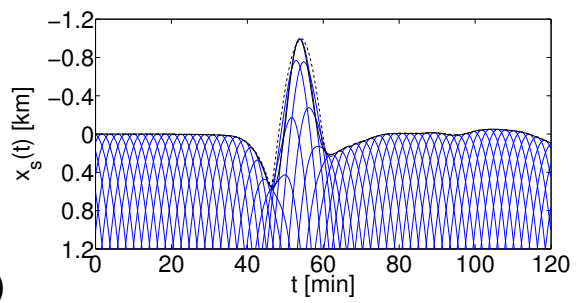

Figure 3.17: Shoreline movement in the Aceh case for the linear model (a: LSWE; $\boldsymbol{b}$ : $L V B M)$ coupled to NSWE (dashed line), and for the linear model with an EBC implementation (solid line). Paths of the first-order characteristic curves are shown by thin lines.

(a)

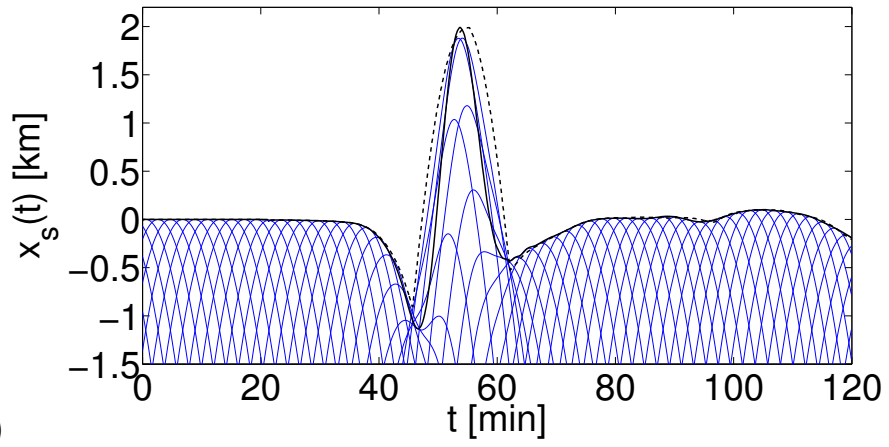

(b)

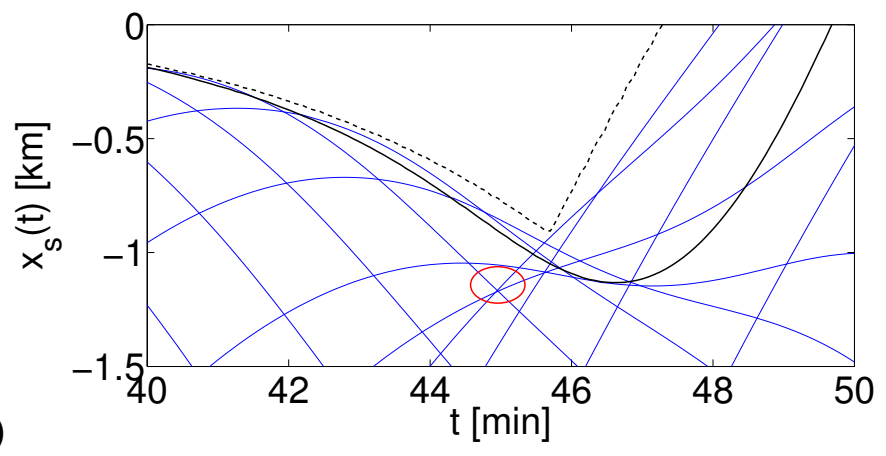

Figure 3.18: Shoreline movement (a) and an inset (b) of a breaking wave simulation. The linear model coupled to NSWE is shown by the dashed line, while the solid one is the shoreline movement of a linear model simulation with EBC implementation. Paths of the first-order characteristic curves are shown by thin lines. Breaking occurs when two incoming characteristic curves intersect before reaching the shoreline. It is indicated by the red oval at approximately $t=45 \mathrm{~min}$. 

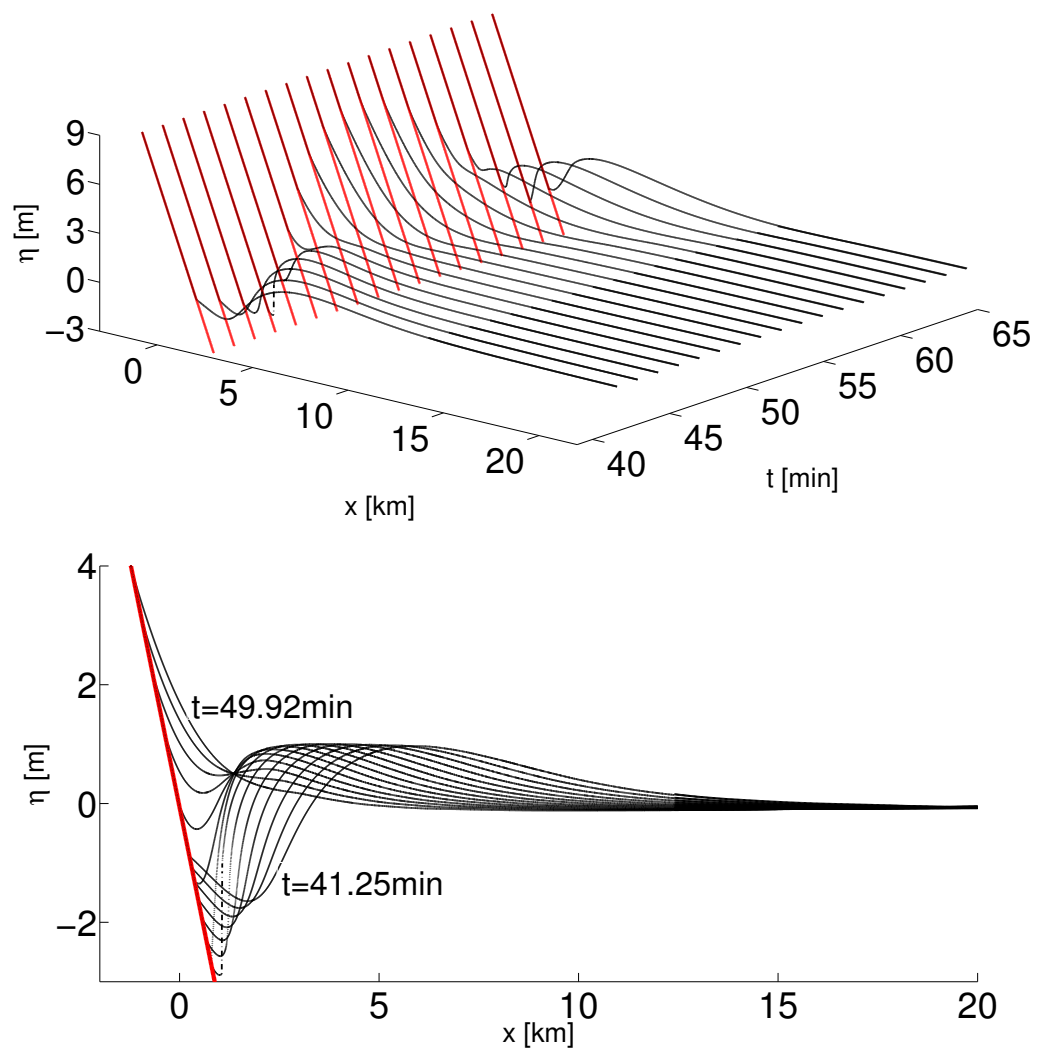

Figure 3.19: Free-surface profiles of a breaking wave simulation for the linear model coupled to the NSWE (dashed and dotted-dashed lines) and for the linear model with an $E B C$ implementation (solid line) at $t=40-70 \mathrm{~min}$. The solid and dashed lines are on top of one another. 


\subsection{Conclusions}

We have formulated a so-called effective boundary condition (EBC), which is used as an internal boundary condition within a domain divided into simulation and model areas. The simulation area from the deep ocean up to a certain depth at a seaward boundary point at $x=B$ is solved numerically using the linear shallow water equations (LSWE) or the linear variational Boussinesq model (LVBM). The nonlinear shallow water equations (NSWE) are solved analytically in the model area from this boundary point towards the coastline over linearly sloping bathymetry. The wave elevation at the seaward boundary point is decomposed into the incoming signal and the reflected one, as described in Antuono and Brocchini (2007, 2010). The advantages of using this EBC are the ability to measure the incoming wave signal at the boundary point $x=B$ for various shapes of incoming waves, and thereafter to calculate the wave run-up and reflection from these measured data. To solve the tsunami wave run-up in the nearshore area analytically, we employ the asymptotic technique for solving the NSWE over sloping bathymetry derived by Antuono and Brocchini [2010], applied to any given wave signal at $x=B$.

The EBC implementation has been verified in several test cases by comparing simulations in the whole domain (using numerical solutions of the LSWE/ LVBM in the simulation area coupled to the NSWE in the model area) with ones using the EBC. We have also validated our approach with the laboratory experiment of Synolakis 1987 for the run-up of a solitary wave over a plane beach. The location of the boundary point $x=B$ is considered before the nonlinearity plays an important role in the wave propagation. The comparisons between both simulations show that the EBC method gives a good prediction of the wave run-up as well as the wave reflection, based only on the information of the wave signal at this seaward boundary point. It is also shown that the EBC technique can capture the resonance effect that occurs due to the incoming and reflected wave interactions. The computational times needed for simulations using the EBC implementation show a large reduction compared to times required for the corresponding full numerical simulations. Hence, without losing the accuracy of the results, we could compress the time needed to simulate wave dynamics in the nearshore area.

An extension of this EBC technique to the case where the NSWE model is used both in the simulation and the model areas follows directly from the variational methodology. The analytical benchmark for this case is provided by Carrier et al. [2003] and Kânoğlu [2004]. The two-dimensional (2D) extension of this technique can be formulated asymptotically using an approach by Ryrie (1983]. For waves incident at a small angle to the beach normal, the onshore problem 
can be calculated using the analytical 1D run-up theory of the nonlinear model, and independently, the longshore velocity can be computed asymptotically. By using a 2D linear model in the open sea towards the seaward boundary line (i.e., in the simulation area), and by employing this approach in the model area, we can in principle apply the EBC method for this 2D case as well. This will be approximately valid for $2 \mathrm{D}$ flow with slow variations along the EBC line. The EBC formulation for the case when the shoreline is fronted by a vertical wall as presented by Kânoğlu and Synolakis [1998 can be obtained by requiring the normal velocity at the shoreline wall boundary to be zero. Another characteristic of the outgoing or reflected waves must be derived (either for the LSWE model or the NSWE model), but the coupling between the numerical and analytical models remains the same as that derived in this article.

\section{Appendix 3.A: Finite volume implementation}

The conservative form of NSWE is given by

$$
\frac{\partial \mathbf{u}}{\partial t}+\frac{\partial \mathbf{f}(\mathbf{u})}{\partial x}=\mathbf{s}
$$

with

$$
\mathbf{u}=\left(\begin{array}{c}
h u \\
h
\end{array}\right) \quad \mathbf{f}(\mathbf{u})=\left(\begin{array}{c}
h u^{2}+\frac{1}{2} g h^{2} \\
h u
\end{array}\right)
$$

and the topographic term

$$
\mathbf{s}=\left(\begin{array}{cc}
-g h & \mathrm{~d} b / \mathrm{d} x \\
0
\end{array}\right)
$$

The system (3.66) is discretized using a Godunov finite volume scheme. First, the domain $[A, B]$ with some fixed $A<x_{\mathrm{s}}(t)$ is partitioned into $N$ grid cells, with grid cell $k$ occupying $x_{k-\frac{1}{2}}<x<x_{k+\frac{1}{2}}$. The Godunov finite volume scheme is derived by defining a space-time mesh with elements $x_{k-\frac{1}{2}}<x<x_{k+\frac{1}{2}}$ and $t_{n}<t<t_{n+1}$, and integrating Eqs. 3.66 over this space-time element:

$$
\begin{aligned}
& \int_{x_{k-\frac{1}{2}}}^{x_{k+\frac{1}{2}}} \mathbf{u}\left(x, t_{n+1}\right) \mathrm{d} x-\int_{x_{k-\frac{1}{2}}}^{x_{k+\frac{1}{2}}} \mathbf{u}\left(x, t_{n}\right) \mathrm{d} x= \\
& \int_{t_{n}}^{t_{n+1}} \mathbf{f}\left(\mathbf{u}\left(x_{k-\frac{1}{2}}, t\right)\right) \mathrm{d} t-\int_{t_{n}}^{t_{n+1}} \mathbf{f}\left(\mathbf{u}\left(x_{k+\frac{1}{2}}, t\right)\right) \mathrm{d} t+\int_{t_{n}}^{t_{n+1}} \int_{x_{k-\frac{1}{2}}}^{x_{k+\frac{1}{2}}} \mathbf{s} \mathrm{d} x \mathrm{~d} t .
\end{aligned}
$$


In the grid cells, we define the mean cell average $\mathbf{U}_{k}=\mathbf{U}_{k}(t)$ as

$$
\mathbf{U}_{k}(t):=\frac{1}{h_{k}} \int_{x_{k-\frac{1}{2}}}^{x_{k+\frac{1}{2}}} \mathbf{u}(x, t) \mathrm{d} x,
$$

with cell length $h_{k}=x_{k+\frac{1}{2}}-x_{k-\frac{1}{2}}$. The function $\mathbf{U}_{k}$ is piecewise constant in each cell. A numerical flux $\mathbf{F}$ is defined to approximate the flux $\mathbf{f}$ :

$$
\mathbf{F}\left(\mathbf{U}_{k}^{n}, \mathbf{U}_{k+1}^{n}\right) \approx \frac{1}{\Delta t} \int_{t_{n}}^{t_{n+1}} \mathbf{f}\left(\mathbf{u}\left(x_{k+\frac{1}{2}}, t\right)\right) \mathrm{d} t .
$$

By using Eqs. 3.70-3.71), expression 3.69 then becomes

$$
\begin{array}{r}
\mathbf{U}_{k}^{n+1}=\mathbf{U}_{k}^{n}-\frac{\Delta t}{h_{k}}\left(\mathbf{F}\left(\mathbf{U}_{k}^{n}, \mathbf{U}_{k+1}^{n}\right)-\mathbf{F}\left(\mathbf{U}_{k-1}^{n}, \mathbf{U}_{k}^{n}\right)\right) \\
+\frac{1}{h_{k}} \int_{t_{n}}^{t_{n+1}} \int_{x_{k-\frac{1}{2}}}^{x_{k+\frac{1}{2}}} \mathbf{s} \mathrm{d} x \mathrm{~d} t .
\end{array}
$$

which is a forward Euler explicit method.

To ensure that the depth is non-negative and that the steady state of a fluid at rest is preserved, the approach of Audusse et al. 2004] is used. The numerical flux $\mathbf{F}$ is then defined as

$$
\mathbf{F}\left(\mathbf{U}_{k}^{n}, \mathbf{U}_{k+1}^{n}\right)=\mathbf{F}_{k+\frac{1}{2}}\left(\mathbf{U}_{\left(k+\frac{1}{2}\right)^{-}}^{n}, \mathbf{U}_{\left(k+\frac{1}{2}\right)^{+}}^{n}\right)
$$

where the interface values are given by

$$
\begin{aligned}
& \mathbf{U}_{\left(k+\frac{1}{2}\right)-}^{n}=\left(\begin{array}{c}
h_{\left(k+\frac{1}{2}\right)-} u_{k} \\
h_{\left(k+\frac{1}{2}\right)-}
\end{array}\right) \text { and } \\
& \mathbf{U}_{\left(k+\frac{1}{2}\right)+}^{n}=\left(\begin{array}{c}
h_{\left(k+\frac{1}{2}\right)+} u_{k+1} \\
h_{\left(k+\frac{1}{2}\right)+}
\end{array}\right) .
\end{aligned}
$$

The topographic term $\mathbf{s}$ is discretized as

$$
\int_{t_{n}}^{t_{n+1}} \int_{x_{k-\frac{1}{2}}}^{x_{k+\frac{1}{2}}} \mathbf{s} \mathrm{d} x \mathrm{~d} t \approx S_{k}=\Delta t\left(\begin{array}{c}
\frac{1}{2} g h_{\left(k+\frac{1}{2}\right)^{-}}^{2}-\frac{1}{2} g h_{\left(k-\frac{1}{2}\right)^{+}}^{2} \\
0
\end{array}\right),
$$

with the water depths $h_{\left(k+\frac{1}{2}\right)^{-}}$and $h_{\left(k+\frac{1}{2}\right)^{+}}$chosen as follows, to ensure the nonnegativity of these depths:

$$
\begin{aligned}
& h_{\left(k+\frac{1}{2}\right)^{-}}=\max \left(h_{k}+b_{k}-b_{k+\frac{1}{2}}, 0\right), \\
& h_{\left(k+\frac{1}{2}\right)^{+}}=\max \left(h_{k+1}+b_{k+1}-b_{k+\frac{1}{2}}, 0\right),
\end{aligned}
$$


and

$$
b_{k+\frac{1}{2}}=\max \left(b_{k}, b_{k+1}\right) .
$$

The discretization of the shallow water equations thus reads

$$
\begin{aligned}
\mathbf{U}_{k}^{n+1}=\mathbf{U}_{k}^{n}-\frac{\Delta t}{h_{k}} & \left(\mathbf{F}_{k+\frac{1}{2}}\left(\mathbf{U}_{\left(k+\frac{1}{2}\right)^{-}}^{n}, \mathbf{U}_{\left(k+\frac{1}{2}\right)^{+}}^{n}\right)\right. \\
& \left.-\mathbf{F}_{k-\frac{1}{2}}\left(\mathbf{U}_{\left(k-\frac{1}{2}\right)^{-}}^{n}, \mathbf{U}_{\left(k-\frac{1}{2}\right)^{+}}^{n}\right)\right)+\frac{\Delta t}{h_{k}} S_{k} .
\end{aligned}
$$

The Harten-Lax-van Leer (HLL) flux Harten et al., 1983, Toro et al., 1994 is used as the numerical flux. It is given by

$$
\mathbf{F}_{k+\frac{1}{2}}^{\mathrm{HLL}}= \begin{cases}\mathbf{F}_{\mathrm{L}} & \text { if } 0<S_{\mathrm{L}} \\ \frac{S_{\mathrm{R}} \mathbf{F}_{\mathrm{L}}-S_{\mathrm{L}} \mathbf{F}_{\mathrm{R}}+S_{\mathrm{L}} S_{\mathrm{R}}\left(U_{\mathrm{R}}-U_{\mathrm{L}}\right)}{S_{\mathrm{R}}-S_{\mathrm{L}}} & \text { if } S_{\mathrm{L}} \leq 0 \leq S_{\mathrm{R}} \\ \mathbf{F}_{\mathrm{R}} & \text { if } 0>S_{\mathrm{R}}\end{cases}
$$

The wave speeds $S_{\mathrm{L}}$ and $S_{\mathrm{R}}$ are approximated as the smallest and largest eigenvalues at the corresponding node. To ensure the stability of this explicit scheme, a Courant-Friedrichs-Lewy (CFL) stability condition per cell is used for all eigenvalues $\lambda_{p}$ at each $\mathbf{U}_{k}^{n}$ :

$$
\left|\frac{\Delta t}{h_{k}} \lambda_{p}\left(\mathbf{U}_{k}^{n}\right)\right| \leq 1
$$

\section{Appendix 3.B: Coupled model}

The (continuous Galerkin) finite element implementation of LSWE or LVBM uses linear polynomial for solving $\phi, \psi$, and $\eta$ approximately, while the finite volume implementation for NSWE approximates $h$ and $u$ with a constant value. Since $u=\partial_{x} \phi$, the velocity of the two models is approximated with the same order of polynomials. By coupling both models, in the simulation area, we can rewrite Eq. (3.52) as

$$
\begin{array}{r}
\mathbf{M}_{\mathrm{kl}} \dot{\eta}_{l}-\mathbf{S}_{\mathrm{kl}} \phi_{l}-\mathbf{B}_{\mathrm{kl}} \psi_{l}-\left.\delta_{k 1}(h u)\right|_{x=B^{-}}=0 \\
\mathbf{M}_{\mathrm{kl}} \dot{\phi}_{k}+g \mathbf{M}_{\mathrm{kl}} \eta_{k}=0 \\
\mathbf{A}_{\mathrm{kl}} \psi_{l}+\mathbf{B}_{\mathrm{kl}} \phi_{l}+\mathbf{G}_{\mathrm{kl}} \psi_{l}-\left.\delta_{k 1}\left(\frac{\tilde{\beta}}{h_{\mathrm{b}}} h u\right)\right|_{x=B^{-}}=0 .
\end{array}
$$

In the finite volume implementation, the boundary condition is inserted through the numerical flux at $x=B$ by using the coupling condition (3.16) as follows:

$$
\left(\begin{array}{c}
h u \\
h
\end{array}\right)=\left(\begin{array}{c}
h_{\mathrm{b}} \partial_{x} \breve{\phi}+\breve{\beta} \partial_{x} \breve{\psi} \\
h_{\mathrm{b}}+\breve{\eta}
\end{array}\right) .
$$




\section{Acknowledgements}

We are pleased to acknowledge funding for W.K. from the Netherlands Organization of Scientific Research (NWO), Earth Sciences division. The idea to couple potential flow (dispersive) models in a simulation area with the full NSWE in the onshore area came originally from the late Prof. Howell Peregrine, and was used by Vijaya Ambati, O. B., and Frank Klaver Klaver, 2009. 


\section{CHAPTER 4}

\section{Conclusions and Outlook}

\subsection{Conclusions}

The effective boundary condition (EBC) for the nondispersive, linear, shallow water equations (LSWE) and the dispersive, linear, variational Boussinesq model (LVBM) has been formulated in this thesis. The EBC is designed as a (partially) reflective, history-dependent internal boundary condition within a domain that is divided into a simulation area and a model area. The wave propagation in the simulation area is modeled numerically over a realistic bathymetry, while the wave dynamics in the model area towards the shoreline and the reflection caused by the bathymetry are modeled analytically. By implementing this technique, fast forecasting of tsunamis can be done and the point-wise wave height in the whole domain (offshore and onshore) area is predicted accurately.

The EBC formulation is started in Chapter 2 by employing the analytical solution of linear shallow water equations in the model area. Flat bathymetry with a closed wall boundary condition is considered as the simplest case. On the one hand, the comparison of the EBC implementation for the LSWE with numerical simulations in the whole domain shows exact agreement in both the wave height and velocity profiles, as shown in Fig. 2.4. On the other hand, the EBC implementation for the LVBM gives the same wave velocity for the first peak wave, while the rest of the waves reveal some differences relative to the LSWE results. This result is expected since a non-dispersive analytical model is used in the model area and only the phase speed of the most dominant wave in the spectrum is chosen. 
Further, the case with slowly varying bathymetry is considered in the model area. Accordingly, the analytical solution is based on the Wentzel-KramerBrillouin (WKB) approximation, as well as extensions for the dispersive Boussinesq model. Comparisons of the numerical performance of simulations in the whole domain and simulations with the EBC implementation show competitive results in several cases. The implementation of the EBC reduces the simulation time up to $30 \%$ as shown in Table 2.2. Meanwhile, comparisons of the LSWE and LVBM show that dispersion effects play an important role in simulations of tsunami propagation. As can be seen in Fig. 2.20, the LVBM simulation yields a higher peak wave elevation of about $1 \mathrm{~m}$. In more dispersive cases, successive wave tails behind the main wave may enhance the tsunami oscillations through resonance.

In Chapter 3, the analytical (asymptotic) solution of the nonlinear shallow water equations (NSWE) is used in the model area for the EBC formulation. The analytical run-up heights at the shore and the reflection caused by the slope are calculated by approximating the bathymetry in the coastal region as a planar beach. Verifications of the results are done by coupling the LSWE and LVBM in the simulation area with a numerical solution of the NSWE in the model area. Comparisons between simulations with the EBC implementation and numerical simulations for the whole domain give a good agreement of the wave run-up as well as the wave reflection. The simulation results also show that when a tsunami wave is dispersive, the oscillations also appear in the shoreline movement as shown in Fig. 3.7. It is also displayed that using the EBC technique, we can capture the resonance effect that occurs due to the incoming and reflected wave interactions. The run-up amplification due to the resonance is strongly dependent on the frequency of the incident wave and influenced by the bathymetry profile. Fig. 3.13 displays the run-up height of an incident wave with an amplitude of $1 \mathrm{~m}$ which amplifies up to $10.67 \mathrm{~m}$ high. Furthermore, the computational times needed in simulations using the EBC implementation show a large reduction up to $97 \%$ compared to times required for corresponding full numerical simulations. The expensive computational time of the full numerical solution is caused by the fine mesh size required in the shore area to get an accurate result of the NSWE. As a consequence, it limits the overall time step, although the mesh size used to solve the LSWE and LVBM in the simulation area is similar to the one with an EBC implementation. Hence, by applying the EBC, we could reduce the time needed to simulate wave dynamics in the nearshore area without losing the accuracy of the results. 

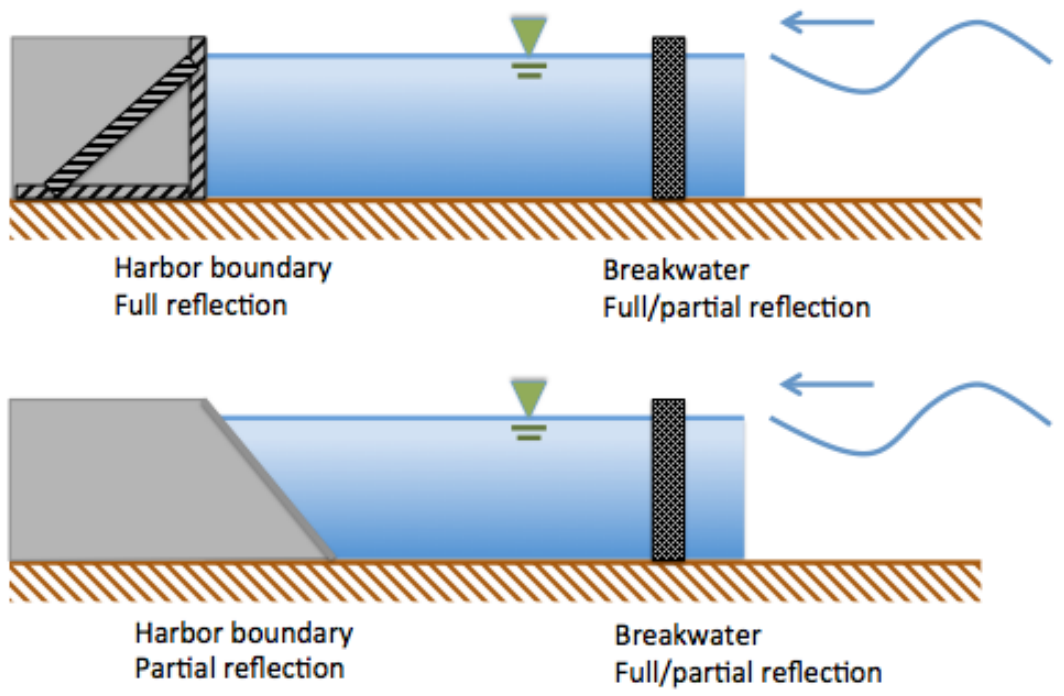

Figure 4.1: Illustration of a simulation of harbor waves.

\subsection{Outlook}

Suggestions for further research are as follows:

- The breaking effects in tsunami waves is ignored in the present analytical solution of nonlinear shallow water equations. This can be improved by incorporating a shock solution in the coastal zone (e.g. Antuono 2010, 2011).

- The present EBC implementations are done in one horizontal dimension approach, using a model area as well as a simulation area. Integration of $2 \mathrm{D}$ numerical modeling in the simulation area with a 1D analytical calculation in the (horizontal) coastal area can be extended directly under certain limitations, for example the negligence of $2 \mathrm{D}$ effects such as refraction, focusing, etc. in the model area.

- Another recommendation for an extension in two horizontal dimensions concerns waves incident at a small angle to the beach normal. Thus, the onshore problem can be calculated using the analytical 1D run-up theory of the nonlinear model, and independently the longshore velocity can be computed asymptotically by using the approach of Ryrie [1983. By implementing 2D numerical modeling in the open sea towards the seaward 
boundary line and employing this approach in the nearshore area, in principle the EBC method for in two horizontal dimensions can be applied as well. This will be approximately valid for horizontal flow with slow variations along the EBC line.

- As mentioned before, the EBC is designed as a (partially) reflective and history-dependent internal boundary condition. In general, the EBC can be used for tsunami simulations, also for the simulation of wind waves from the ocean and sea to include the reflection from the coastal boundaries and coastal structures, such as harbors (O'Sullivan 1992, Pinheiro et al. [2009]), where there are permeable structures like breakwaters and harbor boundaries as illustrated in Fig. 4.1 . 
M. Antuono. A shock solution for the nonlinear shallow water equations. J. Fluid Mech, 658:167-187, 2010.

M. Antuono. Shock trains on a planar beach. J. Fluid Mech, 686:583-606, 2011.

M. Antuono and M. Brocchini. The boundary value problem for the nonlinear shallow water equations. Studies in Applied Mathematics, 119:73-93, 2007.

M. Antuono and M. Brocchini. Solving the nonlinear shallow-water equations in physical space. J. Fluid Mech, 643:207-232, 2010.

E. Audusse, F. Bouchut, M. O. Bristeau, R. Klein, and B. Perthame. A fast and stable well-balanced scheme with hydrostatic reconstruction for shallow water flows. SIAM J. of Sci. Comp., 25(6):2050-2065, 2004.

C. Bender and S. Orszag. Advanced Mathematical Methods for Scientists and Engineers. McGraw-Hill, USA, 1978.

E. N. Bernard and A. R. Robinson. Introduction: Emergent Finnings and New Directions in Tsunami Science. In E. N. Bernard and A. R. Robinson, editors, The Sea, Volume 15: Tsunamis, pages 1-22. Harvard University Press, 2009.

O. Bokhove. Flooding and Drying in Discontinuous Galerkin Finite-Element Discretizations of Shallow-Water Equations. Part 1: One Dimension. J. Sci. Comp., 22:47-82, 2005.

H. Bremmer. The WKB approximation as the first term of a geometrical-optical series. The Theory of Electromagnetic Waves, A Symposium, pages 169-179, 1951. 
M. Brocchini and D. H. Peregrine. Integral flow properties of the swash zone and averaging. J. Fluid Mech, 317:241-273, 1996.

G. F. Carrier and H. P. Greenspan. Water waves of finite amplitude on a sloping beach. J. Fluid Mech, 4:97-109, 1958.

G. F. Carrier, T. T. Wu, and H. Yeh. Tsunami runup and drawdown on a plane beach. J. Fluid Mech, 475:79-99, 2003.

B. H. Choi, V. Kaistrenko, K. O. Kim, B. I. Min, and E. Pelinovsky. Rapid forecasting of tsunami runup heights from 2-D numerical simulations. Nat. Hazards Earth Syst. Sci., 11:707-714, 2011.

B. H. Choi, E. Pelinovsky, K. O. Kim, and B. I. Min. Estimation of Runup Heights of the 2011 off the Pacific Coast of Tohoku Earthquake Tsunami Based on Numerical Simulations. The Open Oceanography J., 6:5-13, 2012.

C. Cotter and O. Bokhove. Water wave model with accurate dispersion and vertical vorticity. Peregrine Commemorative Issue J. Eng. Maths., 67:33-54, 2010 .

I. Didenkulova and E. Pelinovsky. Run-up of long waves on a beach: the influence of the incident wave form. Oceanology, 48:1-6, 2008.

A. Ezersky, D. Tiguercha, and E. Pelinovsky. Resonance phenomena at the long wave runup on the coast. Nat. Hazard Earth Sys., 13:2745-2752, 2013.

E. Gagarina, J. J. W. van der Vegt, and O. Bokhove. Horizontal circulation and jumps in Hamiltonian wave models. Nonlinear. Proc. Geoph., 20:483-500, 2013 .

A. E. Gill. Atmosphere-Ocean Dynamics. Academic Press, 1982.

S. T. Grilli, M. Ioualalen, J. Asavanant, and F. Shi. Source Cconstraints and Model Simmolation of the December 26, 2004, Indian Ocean Tsunami. $J$. Waterw. Port C-ASCE, 133:414-428, 2007.

A. Harten, P. D. Lax, and B. van Leer. On upstream differencing and Godunovtype schemes for hyperbolic conservation laws. SIAM Review, 25:35-61, 1983.

P. Heinrich, F. Schindele, S. Guiborg, and P. F. Ihmle. Modeling of the February 1996 Peruvian tsunami. Geophys. Res. Lett., 25(14):2687-2690, 1998.

E. J. Hinch. Perturbation Methods. Cambridge University Press, 1991. 
J. Horrillo, Z. Kowalik, and Y. Shigihara. Wave Dispersion Study in the Indian Ocean-Tsunami of December 26, 2004. Mar. Geod., 29(3):149-166, 2006.

J. Horrillo, W. Knight, and Z. Kowalik. Tsunami propagation over the North Pacidic: dispersive and nondispersive models. Sci. Of Tsunami Hazards, 31 (3):154-177, 2012.

F. Imamura, A. C. Yalciner, and G. Ozyurt. Tsunami Modeling Manual (TUNAMI Model). 2006.

U. Kânoğlu. Nonlinear evolution and runup and rundown of long waves over a sloping beach. J. Fluid Mech., 513:363-372, 2004.

U. Kânoğlu and C. E. Synolakis. Long wave runup on a piecewise linear topographies. J. Fluid Mech., 374:1-28, 1998.

Y. Kawata, Y. Tsuji, Y. Sugimoto, H. Hayashi, H. Matsutomi, Y. Okamura, I. Hayashi, H. Kayane, Y. Tanioka, K. Fujima, F. Imamura, M. Matsuyama, T. Takahashi, N. Maki, and S. Koshimura. Comprehensive analysis of the damage and its impact on coastal zones by the 2004 Indian Ocean tsunami disaster. 2005. URL http://www.tsunami.civil.tohoku.ac.jp/sumatra2004/ report.html.

A. B. Kennedy and J. T. Kirby. An unsteady wave driver for narrow-banded waves: Modeling nearshore circulation driven by wave groups. Coastal Eng., 48(4):257-275, 2003.

G. Kim, C. Lee, and K. D. Suh. Internal generation of waves: Delta source function method and source term addition method. Ocean Engineering, 34: 2251-2264, 2007.

J. T. Kirby. Nonlinear, dispersive long waves in water of variable depth. In J. N. Hunt, editor, Gravity Waves in Water of Finite Depth, pages 55-125. Computational Mechanics Publications, 1997.

F. Klaver. Coupling of numerical models for deep and shallow water. Master's thesis, University of Twente, The Netherlands, 2009.

G. Klopman, M. W. Dingemans, and E. van Groesen. A variational model for fully non-linear water waves of Boussinesq type. Proc. $20^{\text {th }}$ Int. Workshop on Water Waves and Floating Bodies, Longyearbyen, Spitsbergen, Norway, 2005.

G. Klopman, E. van Groesen, and M. Dingemans. A variational approach to Boussinesq modelling of fully non-linear water waves. J. Fluid Mech., 657: 36-63, 2010. 
W. Kristina, E. Van Groesen, and O. Bokhove. Effective coastal boundary conditions for dispersive tsunami propagation. Internal report, University of Twente, 2012. URL http://eprints . eemcs .utwente.nl/21961/.

E. Kulikov. Dispersion of the Sumatra Tsunami waves in the Indian Ocean detected by satellite altimetry. Russian J. of Earth Sci., 8,ES4004, 2006.

C. Lee, Y-S. Cho, and K. Yum. Internal generation of waves for extended Boussinesq equations. Coastal Eng., 42:155-162, 2001.

Y. Li and F. Raichlen. Solitary wave runup on plane slopes. J. of Waterway, Port, Coastal, and Oc. Eng., 127:33-44, 2001.

S. L. Lie, D. Adytia, and E. van Groesen. Embedded wave generation for dispersive surface wave models. Ocean Engineering, 80:73-83, 2014.

P. L. F. Liu, Y. B. Cho, S. B. Yoon, and S. N. Seo. Numerical simulation of the 1960 Chilean tsunami propagation and inundation at Hilo, Hawaii. In Y. Tsuchiya and N. Shuto, editors, Tsunami progress in prediction, disaster prevention and warning, pages 99-115. Kluwer Academic Publisher, 1995.

Y. Liu, Y. Shi, D. A. Yuen, E. O. D. Sevre, X. Yuan, and H. L. Xing. Comparison of linear and nonlinear shallow wave water equations applied to tsunami waves over the china sea. Acta Geotechnica, 4:129-137, 2009.

J. C. Luke. A variational principle for fluid with a free surface. J. Fluid Mech., 27:395-397, 1967.

C. L. Mader. Numerical simulation of tsunamis. J. Phys. Oceanogr., 4:74-82, 1974.

C. L. Mader. Numerical Modeling of Water Waves. CRC Press, 2004.

P. A. Madsen and H. A. Schäffer. Analytical solutions for tsunami runup on a plane beach: single waves, N-waves and transient waves. J. Fluid Mech, 645: 27-57, 2010.

P. A. Madsen, R. Murray, and O. R. Sørensen. A new form of the Boussinesq equations with improved linear dispersion characteristics. Part 1. Coast. Eng., 15:371-388, 1991.

C. C. Mei. The applied dynamics of ocean surface waves. World Scientific Publishing Singapore, 1989. 
C. Meinig, S. E. Stalin, A. I. Nakamura, F. Gonzalez, and H. G. Milburn. Technology developments in real-time tsunami measuring, monitoring and forecasting in oceans. MTS/IEEE, 19-23 September 2005, Washington D. C., 2005.

J. W. Miles. On Hamiltonian's principle for surface waves. J. Fluid Mech., 83: 153-158, 1977.

S. Neetu, I. Suresh, R. Shankar, B. Nagarajan, R. Sharma, S. S. C. Shenoi, A. S. Unnikrishnan, and D. Sundar. Trapped waves of the 27 november 1945 Makran tsunami: observations and numerical modeling. Nat. Hazards, 59, 2011.

M. Ortiz, E. Gomez-Reyes, and H. S. Velez-Munoz. A fast preliminary estimation model for transoceanic tsunami propagation. ITS 2001 Proceedings, pages 723739, 2001.

E. J. O'Sullivan. Wave reflection effects within a harbour. Master's thesis, The University of British Columbia, Canada, 1992.

E. N. Pelinovsky and R. KH. Mazova. Exact analytical solutions of nonlinear problems of tsunami wave run-up on slopes with different profiles. Nat. Hazards, 6:227-249, 1992.

D. H. Peregrine. Long waves on a beach. J. Fluid Mech., 27(4):815-827, 1967.

L. Pinheiro, C. J. Fortes, J. A. Santos, and M. Walkley. Implementation of partial reflection boundary conditions in wave propagation model boussiiw. J. Coastal Res., SI 56:1040-1044, 2009.

J. Roger, S. Allgeyer, H. Hebert, M. A. Baptista, A. Loevenbruck, and F. Schindele. The 1755 Lis bon tsunami in Guadeloupe Aarchipelago: source sensitivity and investigation of resonance effects. Open Oceanography J., 4:58-70, 2010.

S. C. Ryrie. Longshore motion generated on beaches by obliquely incident bores. J. Fluid Mech., 129:193-212, 1983.

T. Saito, Y. Ito, D. Inazu, and R. Hino. Tsunami source of the 2011 Tohoku?Oki earthquake, Japan: Inversion analysis based on dispersive tsunami simulations. J. Geoph. Res., 38,L00G19, 2011.

S. Sato. Numerical Simulation of 1993 Southwest Hokkaido Earthquake Tsunami around Okushiri Island. J. Waterway, Port, Coastal, Ocean Eng., 122(5):209$215,1996$. 
F. Shi, J.T. Kirby, B. Tehranirad, J.C. Harris, and S. Grilli. FUNWAVE TVD: Fully Nonlinear Boussinesq Wave Model with TVD Solver Documentation and Users' Manual (Version 1.0). Research Report NO. CACR-11-04, Center for Applied Coastal Research, Ocean Engineering Laboratory, University of Delaware, Newark, 2011.

T. S. Stefanakis, F. Dias, and D. Dutykh. Local run-up amplification by resonant wave interactions. Phys. Rev. Lett., 107:124502, 2011.

C. E. Synolakis. The runup of solitary waves. J. Fluid Mech., 185:523-545, 1987.

C. E. Synolakis and E. N. Bernard. Tsunami science before and after Boxing Day 2004. Phil. Trans. R. Soc. A, 364(1845):2231-2265, 2006.

C. E. Synolakis, E. N. Bernard, V. V. Titov, U. Kânoğlu, and F. I. Gonzáles. Validation and Vreification of Tsunami Numerical Models. Pure Appl. Geophys., 165:2197-2228, 2008.

V. V. Titov, C. W. Moore, D. J. M. Greenslade, C. Pattiaratchi, R. Badal, C. E. Synolakis, and U. Kânoğlu. A new tool for inundation modeling: Community modeling interface for tsunamis (commit). Pure Appl. Geophys., Topical Volume, 2011.

E. F. Toro, M. Spruce, and W. Speares. Restoration of the contact surface in the HLL-Riemann solver. Shock Waves, 4:25-34, 1994.

E. van Groesen. Variational Boussinesq Model, part 1: Basic equations in Cartesian coordinates. Technical Report of LabMath-Indonesia, 2006.

E. van Groesen and Andonowati. Fully dispersive dynamic models for surface water waves above varying bottom, Part 1: Model equations. Wave Motion, 48:657-666, 2011.

E. van Groesen and Jaap Molenaar. Continuum Modelling in the Physical Sciences, in: Mathematical Modeling and Computation. SIAM, 2007.

X. Wang and P. L. F. Liu. Preliminary study of possible tsunami hazards in Taiwan region. Cornell University, 2006.

Y. Wei, E. N. Bernard, L. Tang, R. Weiss, V. V. Titov, C. Moore, M. Spillane, M. Hopkins, and U. Kânoğlu. Real-time experimental forecast of the Peruvian tsunami of August 2007 for U.S. coastlines. Geophys. Res. Lett., 35:L04609, 2008 . 
G. B. Whitham. Variational methods and applications to water waves. Proc. $R$. Soc. London A, 299(1456):6-25, 1997.

A. C. Yalciner, E. N. Pelinovsky, U. Kuran, T. Taymaz, A. Zaitsev, G. Ozyurt, C. Ozer, H. Karakus, and I. Safak. Simulation and Comparison with Field Survey Results of Dec., 26, 2004 Tsunami. 2005. URL http://yalciner.ce. metu.edu.tr/sumatra/survey/simulation/index.htm.

N. Zaibo, E. Pelinovsky, A. Kurkin, and A. Kozelkov. Estimation of far-field tsunami potential for the Caribbean coast based on numerical simulation. Sci. of Tsunami Hazards, 21(4):202-222, 2003.

V. E. Zakharov. Stability of periodic waves of finite amplitude on the surface of a deep fluid. J. Appl. Mech. and Techn. Phys., 9(2):190-194, 1968.

H. Zhang, Y. Shi, D. A. Yuen, Z. Yan, X. Yuan, and C. Zhang. Modeling and Visualization of Tsunamis. Pure Appl. Geophys., 165:475-496, 2008. 



\section{Acknowledgments}

I met both Prof. Brenny van Groesen and Prof. Onno Bokhove for the first time during the Research Workshop of Mathematical and Numerical Modeling of Geophysical Flows, organized by LabMath-Indonesia (LMI), in Bandung, on 17-28 July 2006. I was on the last year of my bachelor study, and never thought before that the workshop would bring me this far. I therefore would like to thank Brenny and Onno for giving me the opportunity to do my master and doctoral degree at the University of Twente as well as the motivation and encouragement during this journey. I am much indebted to Brenny for his patience and dedication in guiding me throughout the entire research project. I am also enormously thankful to Onno for his guidance and enthusiasm during these years.

I would like to pronounce my sincere thanks to Prof. Stephan van Gils, the chair of AAMP, and Prof. Jaap van der Vegt, the chair of MACS, for all the supports when I worked at their group. I thank the other members of my graduation committee, Prof. Peter Apers, Prof. Harry Hoeijmakers, Prof. Rene Huijsmans, Prof. Hans Kuerten, and Prof. Harald van Brummelen, for agreeing to be in the committee and for reading the final draft of my thesis. I also appreciate Dr. Gerard Jeurnink for involving me as his teaching assistant in several classes.

Part of this thesis was also done at LMI, and I wish to express my thanks to Dr. Andonowati for providing a nice research atmosphere and always letting me to work at LMI. During my stay there, I met many people with whom I can discuss about everything. I thank Ardhasena Sopaheluwakan, Didit Adytia, Andreas, Hafizh, Meirita, and Mourice. I am also indebted to Agus Suryanto and Isnani Darti, my bachelor supervisors, for giving me the foundation in applied mathematics and also for introducing me to Brenny.

A special word of thanks goes to the secretaries Mariëlle, Linda, and Carin at Twente, as well as Dian and Mira at LMI, for all the administrative arrangements. 
It must be difficult for me to work within the group without the support and presence of my friends. Thanks to everyone I have shared my office with: Arnida, Elena (for a long-lasting office mate), Ellen, Bob, David, Masoumeh, Milan, Sander, Shavarsh. I would like also to thank the other members of the group: Alyona, Anastasia, Anthony, Bettina, Briti, Deepak, Edo, Henk, Huan, Ruddy, Liam, Lulu, Ivan, Julia, Lilya, Marcel, Sid, Tatyana, Thomas, Tim, Tito, and Vijaya.

Being far away from my family is not easy, yet I am really grateful for being surrounded by many warmhearted people. I thank tante Soefiyati Hardjosumarto and Ingrid Proost for countless invitations to their house. I also thank oom Dick and tante Lieke Biver in Leiden, and oom Frank and tante Wita Mertes (also Felix and Laura) in Bad Neunahr, Germany for their thoughtfulness. I wish to express my thanks to David Marpaung and Vince, Januar, Jenny Ngo, Husna, Pythia, Santy, Stephanus, Stevie, and Tettri. I am also very thankful to all member of Indonesian Student Association in Enschede (PPIE) whom I cannot name one by one, which made my stay in Enschede enjoyable.

Many thanks are due to oom Widjaja Hendra and tante Milka for their continuous prayer and care. I also thank my brothers and sisters, Andri and Ling, Renny and Setia, for their support. I am truly grateful to my mother for her invaluable prayer and love. Thank you for teaching me everything, including mathematics since I was child. Finally, I thank my husband for his love, patience, and understanding during all the ups and downs of this journey, and for the encouragement to finish this thesis. Above all, I thank God for His grace and faithfulness in my life. 
ISBN: 978-90-365-3755-1 